\title{
Search for long-lived, massive particles in events with a displaced vertex and a muon with large impact parameter in pp collisions at $\sqrt{s}=13$ TeV with the ATLAS detector
}

\author{
G. Aad et al. \\ (ATLAS Collaboration)
}

(Received 27 March 2020; accepted 1 July 2020; published 14 August 2020)

\begin{abstract}
A search for long-lived particles decaying into hadrons and at least one muon is presented. The analysis selects events that pass a muon or missing-transverse-momentum trigger and contain a displaced muon track and a displaced vertex. The analyzed dataset of proton-proton collisions at $\sqrt{s}=13 \mathrm{TeV}$ was collected with the ATLAS detector and corresponds to $136 \mathrm{fb}^{-1}$. The search employs dedicated reconstruction techniques that significantly increase the sensitivity to long-lived particle decays that occur in the ATLAS inner detector. Background estimates for Standard Model processes and instrumental effects are extracted from data. The observed event yields are compatible with those expected from background processes. The results are presented as limits at $95 \%$ confidence level on model-independent cross sections for processes beyond the Standard Model, and interpreted as exclusion limits in scenarios with pair production of long-lived top squarks that decay via a small $R$-parity-violating coupling into a quark and a muon. Top squarks with masses up to $1.7 \mathrm{TeV}$ are excluded for a lifetime of $0.1 \mathrm{~ns}$, and masses below $1.3 \mathrm{TeV}$ are excluded for lifetimes between $0.01 \mathrm{~ns}$ and $30 \mathrm{~ns}$.
\end{abstract}

DOI: 10.1103/PhysRevD.102.032006

\section{INTRODUCTION}

The Standard Model (SM) of particle physics has successfully predicted the results of decades of laboratory experiments with impressive precision, but it suffers from several notable inadequacies. For example, the SM lacks explanations for the scale hierarchy of the interactions $[1,2]$ and dark matter [3], and it does not include a quantum description of gravity. However, despite ambitious search programs, the experiments at the Large Hadron Collider (LHC) have not yet reported any evidence of physics beyond the Standard Model (BSM).

A possible way for BSM signatures to evade the constraints from these searches is if the BSM particles produced in proton-proton $(p p)$ collisions do not decay promptly but have lifetimes sufficiently long to yield decay lengths of the order of $1 \mathrm{~mm}$ or more. Such long-lived particles (LLPs) can generate a variety of unconventional detector signatures that are often completely free of irreducible backgrounds from SM processes. However, without dedicated reconstruction algorithms and analysis techniques that consider this possibility, a discovery of LLPs may be missed [4]. While there

*Full author list given at the end of the article.

Published by the American Physical Society under the terms of the Creative Commons Attribution 4.0 International license. Further distribution of this work must maintain attribution to the author(s) and the published article's title, journal citation, and DOI. Funded by SCOAP. is a long history of searches for LLP signatures at colliders, such searches have attracted increased interest since the startup of the LHC [5]. This paper reports a search for decays of LLPs including a muon, using the full Run-2 dataset of the ATLAS experiment.

Extensions of the Standard Model involving supersymmetry (SUSY) [6-11] are appealing from a theoretical perspective, e.g., due to their potential to achieve gaugecoupling unification, provide an explanation for dark matter, and alleviate the naturalness problem [12-15]. Scenarios with a SUSY partner of the top quark, the top squark $\tilde{t}$, with a mass close to the weak scale are of particular interest. This is due to the large quantum corrections to the Higgs boson mass from top-quark loops that are at the center of the naturalness problem [16,17].

The vast majority of searches for the $\tilde{t}$ squark have assumed that $R$ parity is conserved. This quantity is defined as $R_{p} \equiv(-1)^{3(B-L)+2 s}$ where $B, L$, and $s$ denote baryon number, lepton number, and spin, respectively [18]. Apart from small nonperturbative effects $[19,20], B$ and $L$ are conserved in the SM. It is often assumed that their conservation will translate to the SUSY sector to automatically avoid low-energy constraints on $B$ and $L$ violation. However, these conserved quantities in the SM are due to accidental symmetries, not fundamental symmetries. In the minimal supersymmetric extension to the SM (MSSM) $[21,22]$, couplings that violate baryon-number and leptonnumber conservation naturally occur at tree level. The couplings responsible for these violations are collectively 
called $R$-parity-violating (RPV) couplings. The RPV terms of the MSSM superpotential are given by

$$
\mathcal{W}_{\mathrm{RPV}}=\mu_{i} l_{i} h_{u}+\lambda_{i j k} l_{i} l_{j} \bar{e}_{k}+\lambda_{i j k}^{\prime} l_{i} q_{j} \bar{d}_{k}+\lambda_{i j k}^{\prime \prime} \bar{u}_{i} \bar{d}_{j} \bar{d}_{k},
$$

where $\mu_{i}, \lambda_{i j k}, \lambda_{i j k}^{\prime}$, and $\lambda_{i j k}^{\prime \prime}$ are the RPV couplings, $l$ and $e$ represent the lepton and charged-lepton supermultiplets, $h_{u}$ represents the up-type Higgs supermultiplets, and $q, u$, and $d$ represent the quark, up-type quark, and down-type quark supermultiplets, respectively [18]. The symbols $i, j$, and $k$ are generation indices. Nonzero RPV couplings can, for example, result in an unstable lightest SUSY particle (LSP) rendering constraints from many SUSY searches invalid. In such models, the LSP does not serve as a dark-matter candidate as it often does in $R_{p}$-conserving models [23,24].

The search presented here targets nonzero values of the $\lambda_{i j k}^{\prime}$ coupling. All other RPV couplings are assumed to be exactly zero. Various low- and intermediate-energy constraints set upper limits on the size of these $\lambda_{i j k}^{\prime}$ couplings, particularly for couplings involving light flavors. These include measurements of the elements of the CabibboKobayashi-Maskawa matrix, constraints on neutrinoless double-beta decay, and $B$-physics measurements. Many of these constraints suggest that the value of any nonzero RPV coupling needs to be small. In turn, this naturally leads to suppression of the decay processes and can give rise to long-lived SUSY particles. A nonzero $\lambda_{23 k}^{\prime}$ coupling would allow a top squark to decay into a muon and a $k$ thgeneration down-type quark, as shown in Fig. 1. The strongest indirect constraints on this coupling come from partial-width measurements of the $Z$ boson at LEP and exclude $\lambda_{23 k}^{\prime}>0.45$, assuming the existence of a squark with a mass of $100 \mathrm{GeV}$. A summary of experimental constraints on RPV SUSY is given in Ref. [18].

In models with sufficiently small $\lambda_{23 k}^{\prime}$ coupling values and where the $\tilde{t}$ squark is the LSP, the suppression of the decay causes it to occur at discernible distances from the

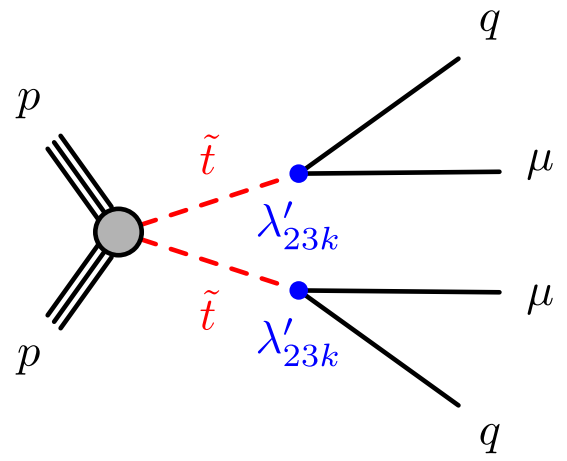

FIG. 1. Diagram showing production of a top squark-antisquark pair (both denoted by $\tilde{t}$ ), in which the top (anti-)squark decays into $\mu^{+}\left(\mu^{-}\right)$and a down-type (anti-)quark of generation $k$. With sufficiently small values of the $R$-parity-violating coupling $\lambda_{23 k}^{\prime}$, the lifetime of the $\tilde{t}$ becomes long enough to give rise to decays which are significantly displaced from their production point. $p p$ interaction point where the $\tilde{t}$ squark pair was produced. This would give rise to muons and high-mass vertices that are significantly displaced from the interaction point, yielding a distinctive detector signature in a collider experiment, with no irreducible backgrounds from SM processes. The search presented here is designed to be sensitive to this signature.

Other proposed BSM scenarios that could result in longlived particle decays into at least one muon include longlived lepto-quarks [25], long-lived BSM particles appearing in decays of Higgs bosons [26,27], scenarios with righthanded neutrinos with Majorana masses below the electroweak scale [28] and RPV scenarios with a long-lived electroweakino LSP decaying through a virtual scalar muon and a $\lambda_{2 j k}^{\prime}$ coupling [29].

Searches for a $\tilde{t}$ squark decaying promptly via the $\lambda_{i j k}^{\prime}$ couplings have been performed by the ATLAS and CMS Collaborations [30,31]. Exclusion limits on long-lived top squarks decaying into a muon and a hadronic jet have also been obtained by the CMS Collaboration, excluding $\tilde{t}$ squark masses below $1.4 \mathrm{TeV}$ for a mean proper lifetime of $0.1 \mathrm{~ns}$ [32-34]. Related searches for displaced lepton production in association with displaced hadronic activity have been performed by the ATLAS Collaboration $[35,36]$.

In the search presented in this paper, dedicated tracking and vertexing algorithms are employed to retain selection efficiency for such LLP signatures. Events are required to contain a reconstructed displaced vertex and a reconstructed muon with a large impact parameter. Unlike previous searches from the ATLAS Collaboration [35,36], the muon is not required to be associated with the vertex, in order to be inclusive of various BSM decay topologies. Signal region criteria are designed to select no more than roughly one background event in the available dataset, while maximizing the expected signal yield. The uncertainty in any background prediction is dominated by the statistical component. The predictions of the background yields are entirely derived in data, with individual contributions estimated from dedicated control regions.

\section{ATLAS DETECTOR}

The ATLAS detector [37] at the LHC is a multipurpose particle detector with a forward-backward symmetric cylindrical geometry and a near $4 \pi$ coverage in solid angle. ${ }^{1}$ It consists of an inner detector (ID) surrounded by a thin

\footnotetext{
${ }^{1}$ ATLAS uses a right-handed coordinate system with its origin at the nominal interaction point (IP) in the center of the detector and the $z$ axis along the beam pipe. The $x$ axis points from the IP to the center of the LHC ring, and the $y$ axis points upwards. Cylindrical coordinates $(r, \phi)$ are used in the transverse plane, $\phi$ being the azimuthal angle around the $z$ axis. The pseudorapidity is defined in terms of the polar angle $\theta$ as $\eta=-\ln \tan (\theta / 2)$. Angular distance is measured in units of $\Delta R \equiv \sqrt{(\Delta \eta)^{2}+(\Delta \phi)^{2}}$.
} 
superconducting solenoid, electromagnetic and hadronic calorimeters, and a muon spectrometer (MS) incorporating three large superconducting toroidal magnets.

The ID is immersed in a $2 \mathrm{~T}$ axial magnetic field and provides charged-particle tracking in the range $|\eta|<2.5$. The high-granularity silicon pixel detector covers the vertex region and typically provides four measurements per track, the first hit being normally in the insertable $B$ layer $[38,39]$ located just outside the beam pipe at a radius of $33 \mathrm{~mm}$ from the proton beam line. Three more concentric pixel layers are situated at radii of $50.5,88.5$ and $122.5 \mathrm{~mm}$ before the first active layer of the semiconductor tracker (SCT) at $r=299 \mathrm{~mm}$. Based on silicon microstrip technology, the SCT typically provides eight separate measurements. These silicon detectors are complemented by the transition radiation tracker (TRT), a straw-tube drift chamber which enables radially extended track reconstruction up to $|\eta|=2.0$.

In the central pseudorapidity range, energy measurements are provided by a high-granularity lead/liquid-argon (LAr) sampling calorimeter for electromagnetic deposits, and a steel/scintillator-tile calorimeter for hadrons $(|\eta|<$ 1.475 and $|\eta|<1.7$, respectively). The end cap and forward regions are instrumented with LAr calorimeters for both the electromagnetic and hadronic energy measurements up to $|\eta|=4$.9. Together, these systems provide full coverage in the region $|\eta|<4.9$.

The MS surrounds the calorimeters and features three large air-core toroidal superconducting magnets with eight coils each. The integral of the magnetic field from the toroids ranges between 2 and $6 \mathrm{~T} \mathrm{~m}$ for particle trajectories across most of the detector. The MS includes a system of precision tracking chambers and fast detectors for triggering. Three layers of drift-tube chambers provide an accurate measurement of the muon track curvature in the region $|\eta|<2.0$, while two layers of drift-tube chambers and one layer of cathode-strip chambers extend this measurement to $|\eta|<2.7$. Resistive-plate chambers provide muon triggering capability for $|\eta|<1.05$ while thin-gap chambers are used in the region $1.05<|\eta|<2.4$.

Interesting events are selected to be recorded by the firstlevel trigger system implemented in custom hardware, followed by selections made by algorithms implemented in software in the high-level trigger [40]. The first-level trigger makes decisions at the $40 \mathrm{MHz}$ bunch crossing rate to keep the event selection rate below $100 \mathrm{kHz}$, which the high-level trigger further reduces in order to record events to disk at about $1 \mathrm{kHz}$.

\section{DATA SAMPLES AND SIMULATED EVENTS}

The analysis is performed on a set of $p p$ collision data at $\sqrt{s}=13 \mathrm{TeV}$ recorded during 2016-2018 which, after requiring good quality of the data [41], corresponds to an integrated luminosity of $136 \mathrm{fb}^{-1}$. The uncertainty in the combined 2015-2018 integrated luminosity is 1.7\% [42], obtained using the LUCID-2 detector [43] for the primary luminosity measurements. The data analyzed for this result were recorded using triggers requiring either a track in the MS with transverse momentum $p_{\mathrm{T}}>60 \mathrm{GeV}$ and $|\eta|<1.05$, or large missing transverse momentum $E_{\mathrm{T}}^{\text {miss }}$ as measured in the calorimeters $\left(E_{\mathrm{T}}^{\text {miss }}>100 \mathrm{GeV}\right.$ for a portion of the 2016 data, and $E_{\mathrm{T}}^{\text {miss }}>110 \mathrm{GeV}$ for the rest of the analyzed dataset). In the recorded events, there are approximately $35 p p$ collisions in the same LHC bunch crossing, on average.

Samples of long-lived top squark-antisquark pairs (denoted by $\tilde{t} \tilde{t}$ ) from Monte Carlo (MC) simulations are used as benchmarks to study expected signal efficiencies. Samples with $1 \mathrm{TeV} \leq m(\tilde{t}) \leq 2 \mathrm{TeV}$ were generated in steps of $100 \mathrm{GeV}$, for mean proper lifetimes $\tau(\tilde{t})$ of $0.01,0.1$ and $1 \mathrm{~ns}$ for each mass value. All other SUSY-particle contributions are assumed to be decoupled. The matrix element calculation for the $\tilde{t}$ squark pair production was performed to leadingorder precision with MadGraph5_aMC@NLO 2.6.1 [44] with up to two additional outgoing partons, while the modeling of parton showers, hadronization and the underlying event was performed by PYTHIA 8.230 [45] with the A14 set of tuned parameters [46]. Parton distribution functions (PDF) from the NNPDF23LO [47] set were used. In the signal models considered in this paper, the $\tilde{t}$ squark lifetime is larger than the hadronization timescale in quantum chromodynamics (QCD). Since the $\tilde{t}$ squark carries color charge, it will undergo a hadronization process in Pythia and form a composite colorsinglet state with SM quarks, an $R$ hadron. Dedicated routines for hadronization of heavy colored particles [48] were used to simulate the hadronization process. The top squarks primarily form mesonlike states $(\tilde{t} \bar{q})$, but approximately $10 \%$ of them form baryonlike states $(\tilde{t} q q)$. Roughly half of the $R$ hadrons formed around the top squarks have nonzero electric charge, and due to the $\tilde{t} \tilde{t}$ production the two $R$ hadrons cannot have electric charges of the same sign.

The $R$ hadrons from Pythia are then propagated through a simulation of the ATLAS detector [49] implemented in GEANT 4 v10.1.3 [50] employing dedicated models of $R$-hadron interactions with the detector material [51-53], which can alter the content of the light-quark system in the $R$ hadron, possibly changing its electric charge as it traverses the detector. At the position of the decay, the $R$ hadron is passed to an instance of Pythia which simulates the decay into finalstate particles using the parton shower model described above. The resulting decay products are then further propagated through the detector simulation, starting from the point where the $R$ hadron decays. Reference [54] contains more complete technical details of the treatment of $R$ hadrons in the simulations. The decay process $\tilde{t} \rightarrow \mu q$ is simulated, where $q$ represents light-flavor, down-type quarks, given by nonzero $\lambda_{231}^{\prime}$ and $\lambda_{232}^{\prime}$ couplings. While the simulated mean proper lifetimes of the $\tilde{t}$ squark are $0.01,0.1$, and $1 \mathrm{~ns}$, in the interpretation of the results, additional lifetime values are evaluated by reweighting events from these samples.

Signal production cross sections are calculated to approximate next-to-next-to-leading order in the strong 
coupling constant, adding the resummation of soft gluon emission at next-to-next-to-leading-logarithm accuracy (approximate NNLO + NNLL) [55-62]. The nominal cross sections and their uncertainties are derived using the PDF4LHC15_mc PDF set, following the recommendations of Ref. [63], and range from $6.8 \pm 0.8 \mathrm{fb}$ at a $\tilde{t}$ mass of $1 \mathrm{TeV}$ to $15 \pm 4$ ab at $2 \mathrm{TeV}$.

Samples of $Z \rightarrow \mu^{+} \mu^{-}$events generated using PowhegBox v1 r2856 [64-67] and PYTHIA 8.186 [68], and reconstructed with the same configuration as the signal samples (see Sec. IV), are used to determine scale factors accounting for small differences in muon identification efficiency between the data and the MC simulation.

All simulated event samples include the effects of pileup, i.e., multiple $p p$ interactions per bunch crossing as well as effects on the detector response due to activity from bunch crossings before or after the one containing the selected event. These effects are modeled by overlaying simulated inelastic $p p$ events generated with PYTHIA 8.186 [68] using the NNPDF23LO set of PDFs [47] and the A3 tune [69] over the original hard-scattering event.

\section{EVENT RECONSTRUCTION}

The standard ATLAS track reconstruction is optimized for the trajectories of charged particles originating from the interaction region, or from decays of short-lived particles such as $b$ hadrons. To reduce the computational complexity, constraints are therefore placed on the transverse and longitudinal impact parameters of track candidates relative to the interaction region $\left(\left|d_{0}\right|<10 \mathrm{~mm}\right.$ and $\left|z_{0}\right|<250 \mathrm{~mm}$, respectively), and their hit multiplicities, to select particles emerging from the initial $p p$ collision. The primary vertex $(\mathrm{PV})$ is defined as the collision vertex with the largest $\sum p_{T}^{2}$ of its associated tracks [70].

In order to reconstruct tracks from the decays of longlived BSM particles, which typically have impact parameters that fall outside the standard constraints, a dedicated track reconstruction is performed using large-radius tracking (LRT) [71]. The LRT uses hits not already associated with tracks reconstructed by the standard tracking algorithm in order to improve the efficiency for tracks not originating from the interaction region. Requirements on the track impact parameters are relaxed to $\left|d_{0}\right|<300 \mathrm{~mm}$ and $\left|z_{0}\right|<1500 \mathrm{~mm}$. Requirements on the number of hits which are allowed to be shared by multiple tracks are also relaxed. Tracks from the standard processing and the LRT processing are treated as a single collection in all subsequent steps of reconstruction, such as in the reconstruction of muons used in this search. Apart from the expanded input ID track collection, muons are reconstructed using the algorithms described in Ref. [72].

A dedicated secondary-vertex reconstruction algorithm for LLP decays is employed to reconstruct displaced vertices (DVs). As input to this vertex reconstruction, only tracks with transverse momentum $p_{\mathrm{T}}>1 \mathrm{GeV}$ and $\left|d_{0}\right|>2 \mathrm{~mm}$ are considered. The $d_{0}$ requirement ensures that most tracks from $p p$ collisions and those produced in decays of short-lived, lower-mass SM particles such as $b$ hadrons, are ignored in DV reconstruction. In order to avoid contamination of the vertex reconstruction with fake tracks, which are reconstructed from spurious combinations of hits, the input-track selection includes several additional requirements. Tracks are required to have at least six SCT hits or at least one pixel hit. A track is rejected if it has fewer than two pixel hits and also completely lacks TRT hits. Due to a larger relative contribution of fake tracks at low momentum, tracks with $p_{\mathrm{T}}<25 \mathrm{GeV}$ are subject to the additional requirements of having at least seven SCT hits, as well as having at least 20 TRT hits if within $|\eta|<1$.6. Since only tracks with $\left|d_{0}\right|>2 \mathrm{~mm}$ are considered, a track from a charged LLP originating at the PV will not be included in the vertex reconstructed from its decay products.

The DV reconstruction begins by finding all possible twotrack seed vertices from all possible pairs of selected tracks. The seed vertices are required to have a fit with $\chi^{2}$ per degree of freedom less than 5. Several additional requirements are then applied to ensure the seed vertex tracks are compatible with the decay of a particle originating from the PV. First, both tracks of the seed vertex are required not to have hits in pixel layers at radii smaller than the position of the seed vertex, and are required to have a hit in the first pixel or SCT layer at larger radius, if expected. If a seed vertex is inside or within several millimeters of a tracker barrel layer, hits in that particular layer are neither forbidden nor required. This avoids penalizing cases where it is unclear which side of the layer the vertex is on. Second, the inner product of the vector sum of the track momenta and the vector pointing from the $\mathrm{PV}$ to the seed vertex is required to be positive, ensuring that the seed vertex is pointing away from the PV.

The collection of all possible two-track seed vertices is then iteratively merged to form $n$-track vertices. Ambiguities due to tracks being compatible with more than one vertex are resolved by comparing their $\chi^{2}$ values in the vertex fits, or by merging the vertices if the distance between their estimated positions is not significant enough. To recover losses due to the tight requirements on the tracks used in the seed-vertex finding, tracks compatible with the reconstructed vertices are also attached even if they have associated hits in lower-radius layers. Extra requirements are made on the $d_{0}$ of the tracks before they are attached to the DV in order to suppress fake tracks from the reconstruction. More details about the secondary-vertexing algorithm can be found in Ref. [73].

In the following, the number of associated tracks for a given $\mathrm{DV}$ is denoted by $n_{\text {Tracks }}^{\mathrm{DV}}$, and its visible invariant mass calculated from the four-momenta of the associated tracks, assuming each track was produced by a charged pion, is denoted by $m_{\mathrm{DV}}$.

The missing transverse momentum of an event is usually defined as the negative vector sum of the transverse 
momenta of all identified physics objects (electrons, photons, muons, jets) calibrated individually. In this analysis, an alternative definition using only topological clusters of energy deposits in the calorimeters is used instead [74]. The clusters are locally calibrated to the hadronic scale, but no object-level information is used to calibrate the clusters. In the following, this is referred to as cluster-based $E_{\mathrm{T}}^{\text {miss }}$. This definition is used because of its similarity to the $E_{\mathrm{T}}^{\text {miss }}$ definition that is used in the trigger system, which is based solely on energy deposits in the calorimeter. The benchmark signal considered in this analysis does not include any genuine missing transverse momentum, but since high- $p_{\mathrm{T}}$ muons are expected to deposit only a small fraction of their energy in the calorimeters through ionization, most of their momentum is unaccounted for, making the $E_{\mathrm{T}}^{\text {miss }}$ trigger efficient for this signature.

\section{EVENT SELECTION}

As the LRT processing is computationally expensive, it cannot be run on all events recorded with the ATLAS detector. Instead, during the prompt processing of the data, a tight selection filters out a subset of the events into a special raw-data stream. As the low-level hit information is ordinarily not stored for analysis, this special raw-data stream is later processed using the dedicated reconstruction configuration described in Sec. IV. This filtering is therefore the first step in this analysis, defining the start of two mutually exclusive trigger-based event selections:

(i) $E_{\mathrm{T}}^{\text {miss }}$-triggered sample: Events recorded with the $E_{\mathrm{T}}^{\text {miss }}$ trigger were required to have cluster-based $E_{\mathrm{T}}^{\text {miss }}>180 \mathrm{GeV}$, in order to ensure that events are close to the plateau of the turn-on curve of the $E_{\mathrm{T}}^{\text {miss }}$ trigger. The $E_{\mathrm{T}}^{\text {miss }}$ trigger threshold varied in the range $100-110 \mathrm{GeV}$ over the period in which the data were recorded.

(ii) Muon-triggered sample: Events recorded with the muon trigger, requiring a track in the MS with $|\eta|<$ 1.05 and no explicit requirement of an ID track, are required to have at least one reconstructed muon with $p_{\mathrm{T}}>60 \mathrm{GeV}$. Reconstructed muons are not required to be matched to a muon that fired the trigger in the filtering step, although a trigger matching requirement is made after events are processed with special reconstruction. For events recorded during 2016 data taking, MS tracks that had a well-matched ID track (without LRT) were required to have $\left|d_{0}\right|>1.5 \mathrm{~mm}$. To make this filter more inclusive, for the events recorded during 20172018 data taking, no requirement on the muon $d_{0}$ was imposed. The cluster-based $E_{\mathrm{T}}^{\text {miss }}$ is required to be less than $180 \mathrm{GeV}$ in order to make the two samples mutually exclusive.

Events that survive the filtering step detailed above are reconstructed with the standard ATLAS reconstruction algorithms and the special reconstruction described in Sec. IV. Events are then subject to additional selection criteria. Muon candidates are required to be reconstructed in both the ID (including LRT tracks) and the MS with $p_{\mathrm{T}}>25 \mathrm{GeV}$ and $|\eta|<2.5$. In order to reject muons that originate from SM particles, the $\left|d_{0}\right|$ of the muon with respect to the $\mathrm{PV}$ is required to be larger than $2 \mathrm{~mm}$. Additionally, muon $\left|d_{0}\right|$ and $\left|z_{0}\right|$ are required to be less than 300 and $500 \mathrm{~mm}$, respectively. These requirements define the muоn preselection.

Additional selection criteria are applied to preselected muons to reject major sources of background. The background sources identified for large- $d_{0}$ muons are cosmicray muons, reconstruction algorithm fakes, and muons from in-flight decays of SM hadrons, especially those containing heavy-flavor quarks.

To reduce the contribution from cosmic rays, events which have activity in the MS on the side opposite to the muon are rejected. The spatial positions of track segments reconstructed in individual muon stations are compared with the expected positions given the momentum direction of the reconstructed muon, assuming infinite momentum. Muons with matching segments on the opposite side of the MS that satisfy $\mid \eta$ (muon momentum $)+\eta$ (segment position $) \mid<0.05$ and $\mid \Delta \phi$ (muon momentum, segment position $)-\pi \mid<0.22$ are rejected. The difference between the $\phi$ and $\eta$ requirements is due to the MS detector geometry and its superior resolution in segment $\eta$. Track segments are used for this veto instead of fully reconstructed muon tracks, which are more likely to fail to be reconstructed as muons not pointing back to the interaction region. Angular corrections are applied to account for the longitudinal displacement of the muon. Muons are also rejected if they are opposite in $\eta$ and in $\phi$ to a region of the MS without detector coverage. This requirement is designed to ensure that if the muon were to pass through the detector like a cosmic-ray muon, it would pass through an instrumented region of the MS and can produce a track segment on the opposite side of the muon spectrometer. This requirement removes $94 \%$ of the residual cosmic muon contribution and around $1 \%$ of the contribution from typical signal samples. Together, these requirements constitute the cosmic-muon veto.

Accidental reconstruction of fake displaced muons, from spurious combinations of hits, can also occur. Such fake muons, however, tend to have poor quality of fit and fewer hits on the track. To reject these fake muon candidates, they are required to be constructed from segments in at least three MS stations and have a quality of fit $\chi^{2} / N_{\text {DoF }}<8$. The latter requirement is kept loose to avoid MS alignment mismodeling effects. These requirements constitute the fake-muon veto.

Muons produced in semileptonic decays of short-lived SM hadrons can also contribute to the background processes for this search. These are primarily decays of hadrons containing heavy-flavor quarks. However, such muons are 

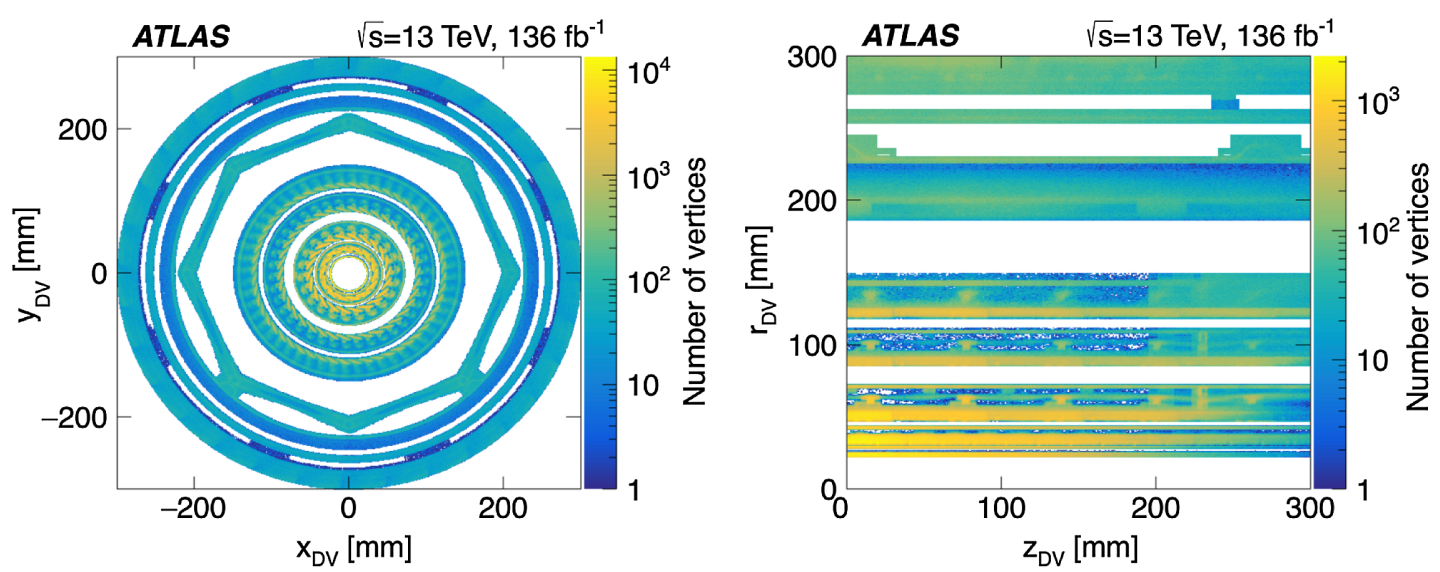

FIG. 2. The positions in the $x-y$ plane (left) and $r-z$ plane (right) of reconstructed displaced vertices that fail the material veto in the analyzed dataset. The latter figure is restricted to positive $z$ values for presentation.

most often produced with nearby energy depositions from hadronic activity. As a result, such processes can be rejected by requiring that the muons are isolated from nearby ID tracks and calorimeter energy deposits. The sum of $p_{\mathrm{T}}$ from tracks consistent with originating at the $\mathrm{PV}$ in a cone of varying size around the muon is required to be no more than $6 \%$ of the muon $p_{\mathrm{T}}$. The cone has a maximum size of $\Delta R=0.3$ for muons with $p_{\mathrm{T}}<33.3 \mathrm{GeV}$, and a cone size of $10 \mathrm{GeV} / p_{\mathrm{T}}$ is used for muons with higher $p_{\mathrm{T}}$. Additionally, the sum of calorimeter cluster $p_{\mathrm{T}}$ in a cone of size $\Delta R=0.2$ around the muon is required to be no more than $6 \%$ of the muon $p_{\mathrm{T}}$. These two requirements define the heavy-flavor veto.

Together, satisfying the cosmic-muon, fake-muon, and heavy-flavor veto criteria constitutes passing the full mиол selection.

The DVs are required to be reconstructed in a cylindrical volume with radius $r_{\mathrm{DV}}<300 \mathrm{~mm}$ and longitudinal extent $\left|z_{\mathrm{DV}}\right|<300 \mathrm{~mm}$, have a fit $\chi^{2} / N_{\text {DoF }}<5$, and have a transverse distance from all reconstructed collision vertices greater than $4 \mathrm{~mm}$. To remain inclusive of other possible signals, there is no explicit requirement that a reconstructed muon be included in the vertex.

To reject vertices arising from interactions with dense detector material, DVs are further required not to have a position consistent with sensitive elements of the detector, its support structures or its services. This veto is imposed via a three-dimensional map of detector material that is constructed from measured positions of low-mass vertices in an inclusive sample and the known positions of detector elements. This veto removes $42 \%$ of the fiducial volume. The positions of reconstructed vertices in the dataset that fail this material veto are shown in Fig. 2. These requirements define the $D V$ preselection. DVs pass the full $D V$ selection if, in addition, they satisfy $n_{\text {Tracks }}^{\mathrm{DV}} \geq 3$ and $m_{\mathrm{DV}}>20 \mathrm{GeV}$, requirements designed to reduce the expected number of background events to around one. For the signal scenarios considered in this paper, roughly half of the DVs satisfying the preselection criteria contain a track from a reconstructed muon, while roughly $80 \%-90 \%$ of DVs passing the full DV selection contain such a track.

Table I lists the preselection and full selection criteria for muons and DVs.

Figure 3 shows the vertex acceptance and efficiency for three benchmark signal scenarios with various mass hypotheses and $\tau(\tilde{t})=1 \mathrm{~ns}$. The vertex acceptance is defined by requiring a $\tilde{t} R$-hadron decay inside the fiducial volume considered by the analysis, $r_{\mathrm{DV}}<300 \mathrm{~mm}, \quad\left|z_{\mathrm{DV}}\right|<$ $300 \mathrm{~mm}$. $R$-hadron decays are further required to decay with a transverse position of $r_{\mathrm{DV}}>4 \mathrm{~mm}$, in order to emulate the requirement that displaced vertices be at least $4 \mathrm{~mm}$ away from any $p p$ collision vertex in the event, in the transverse plane. Stable, electrically charged decay products of the $R$ hadron with $p_{\mathrm{T}}>1 \mathrm{GeV}$ and $\left|d_{0}\right|>2 \mathrm{~mm}$ are used to compute the visible invariant mass and number of visible charged particles leaving the vertex. These requirements

TABLE I. Selection requirements for muons and displaced vertices.

\begin{tabular}{lcc}
\hline \hline Selection level & Muon Selection & Displaced Vertex Selection \\
\hline Preselection & $p_{\mathrm{T}}>25 \mathrm{GeV},|\eta|<2.5$, & $2 \mathrm{~mm}<\left|d_{0}\right|<300 \mathrm{~mm}$, \\
& $\left|z_{0}\right|<500 \mathrm{~mm}$ & $\begin{array}{c}r_{\mathrm{DV}}<300 \mathrm{~mm},\left|z_{\mathrm{DV}}\right|<300 \mathrm{~mm}, \\
\min \left(\left|\vec{r}_{\mathrm{DV}}-\vec{r}_{\mathrm{PV}}\right|\right)>4 \mathrm{~mm}, \chi^{2} / N_{\mathrm{DoF}}<5, \\
\text { Pass material map veto }\end{array}$ \\
Full selection & $\begin{array}{c}\text { Pass cosmic-muon, fake-muon, } \\
\text { and heavy-flavor vetoes }\end{array}$ & $n_{\mathrm{Tracks}}^{\mathrm{DV}} \geq 3$, \\
& $m_{\mathrm{DV}}>20 \mathrm{GeV}$ \\
\hline \hline
\end{tabular}



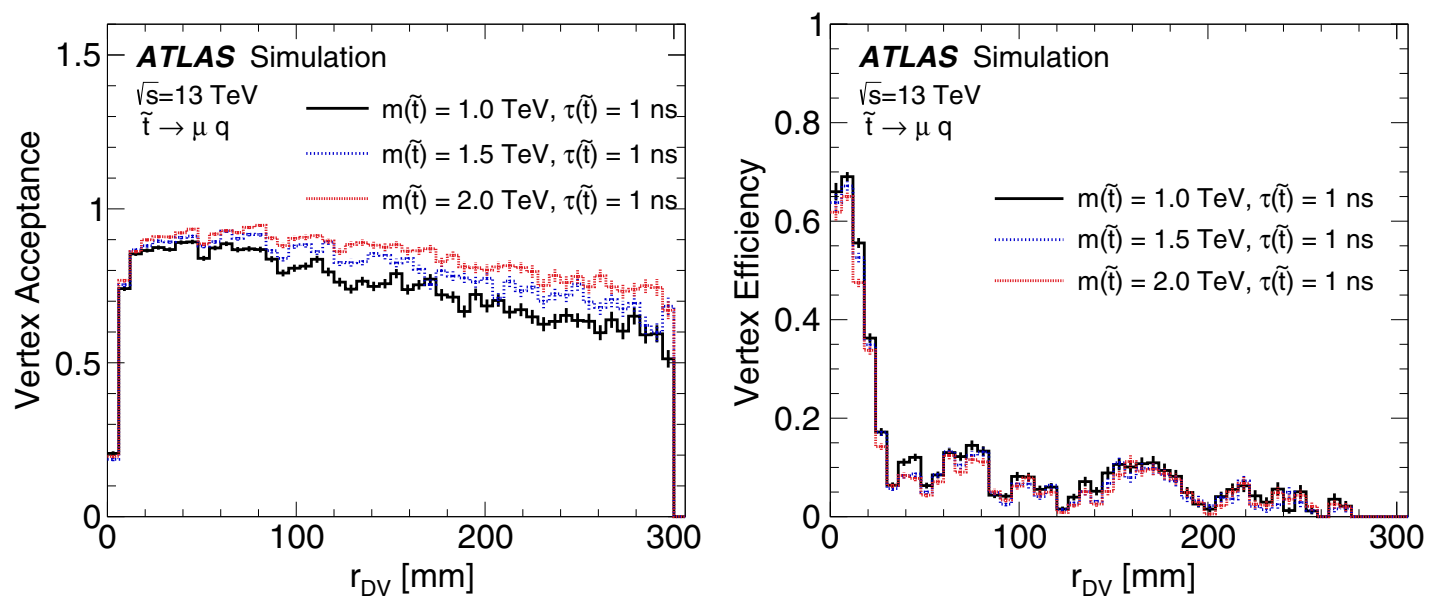

FIG. 3. Vertex selection acceptance (left) and efficiency (right) are shown for the $\tilde{t} R$-hadron benchmark model as a function of the true transverse decay position $r_{\mathrm{DV}}$.

define the tracking acceptance. This visible invariant mass of the vertex is required to be greater than $20 \mathrm{GeV}$, and the vertex must comprise at least three charged particles in the tracking acceptance. The vertexing efficiency is computed with respect to the $R$-hadron acceptance. In order to satisfy the efficiency requirement, $R$-hadron decays must be matched to a reconstructed DV that satisfies the full displaced vertex selection, as described in Table I. The structure in the efficiency distribution as a function of $r_{\mathrm{DV}}$ is largely due to the application of the detector material veto.

For decays that occur inside the fiducial volume, the acceptance varies between $95 \%$ and $55 \%$. The only exception is for decays which occur within millimeters of the primary $p p$ vertex. These decays are either not sufficiently displaced from the primary vertex, or do not produce a sufficient number of charged particles with large transverse impact parameters. Higher acceptance is observed for larger $\tilde{t}$ masses. The vertex efficiency is highest for decays occurring at radii smaller than the beam-pipe radius. For decays which occur at larger radii, the efficiency is affected by the tracking efficiency and material veto. Similar efficiency is observed for different $\tilde{t}$ masses.

The event preselection is applied by requiring that events have at least one preselected muon and a PV with at least two tracks and $\left|z_{\mathrm{PV}}\right|<200 \mathrm{~mm}$. Events in the signal regions (SRs) are required to have at least one fully selected muon and at least one fully selected DV, as described above. Two orthogonal SRs are defined based on the trigger used to record events. The muon trigger $S R$ uses the MS track trigger described above and requires cluster-based $E_{\mathrm{T}}^{\text {miss }}$ below $180 \mathrm{GeV}$ and a muon with $p_{\mathrm{T}}>$ $62 \mathrm{GeV}$ and $|\eta|<1.05$ to ensure efficient triggering. This muon is further required to spatially coincide with the trigger-level muon. The $E_{\mathrm{T}}^{\text {miss }}$ trigger $S R$ uses the $E_{\mathrm{T}}^{\text {miss }}$ based trigger described above and requires that the clusterbased $E_{\mathrm{T}}^{\text {miss }}$ be larger than $180 \mathrm{GeV}$. The event selection requirements are summarized in Table II.

\section{BACKGROUND ESTIMATION}

The backgrounds for displaced muons described in Sec. V are largely removed by dedicated veto requirements. Sources of background for DVs include detector material interactions and randomly intersecting tracks, which are efficiently suppressed by vetoing vertices in regions known to have material and requiring $n_{\text {Tracks }}^{\text {DV }} \geq 3$ and $m_{\mathrm{DV}}>20 \mathrm{GeV}$.

The background estimation used in this analysis relies on the fact that the variables used to reject displaced muons from background sources are uncorrelated with the variables used to reject displaced vertices from background. This is exploited in order to estimate backgrounds in the SR from data. Any residual correlation is measured and treated

TABLE II. Event selection requirements.

\begin{tabular}{lcc}
\hline \hline Selection level & $E_{\mathrm{T}}^{\text {miss }}$ Trigger SR & Muon Trigger SR \\
\hline Preselection & $\begin{array}{c}\text { Selected by } E_{\mathrm{T}}^{\text {miss }} \text { trigger, } \\
\text { Cluster-based } E_{\mathrm{T}}^{\text {miss }}>180 \mathrm{GeV}, \\
\text { Preselected muon }\end{array}$ & $\begin{array}{c}\text { Selected by muon trigger, } \\
\text { Cluster-based } E_{\mathrm{T}}^{\text {miss }}>180 \mathrm{GeV}, \\
\text { Preselected muon, }\end{array}$ \\
Full selection & $\begin{array}{c}\text { Highest- } p_{\mathrm{T}} \text { muon matches a trigger muon } \\
\text { and has } p_{\mathrm{T}}>62 \mathrm{GeV} \text { and }|\eta|<1.05\end{array}$ \\
\hline \hline
\end{tabular}


as a systematic uncertainty in the background prediction as described in Sec. VII. The following subsections describe how the residual backgrounds from cosmic rays, algorithm fakes, and heavy-flavor decays in the SRs are determined.

For each of the sources of background muons, a dedicated control region (CR) is constructed as described in Sec. VIA. Transfer factors for each of these muon backgrounds are measured in regions with background-like DVs and are used to extrapolate from CRs to the SR, as discussed in Sec. VIB. These extrapolations are validated in a set of validation regions (VRs) with an orthogonal set of background-like DVs. The full estimation of the background yield is presented in Sec. VIC.

\section{A. Region definitions}

Preselected events are divided into three regions depending on the DV properties:

(1) DV Control Region ( $D V C R$ ): no DVs passing the preselection, comprising three subregions:

(a) No DVs with a good fit quality passing the material map veto,

(b) Only two-track DVs failing the material map veto,

(c) Only three-track DVs failing the material map veto;

(2) DV Validation Region ( $D V V R$ ): preselected DVs not passing the full selection, comprising two subregions:

(a) Preselected two-track DVs, (b) Preselected DVs with $m_{\mathrm{DV}}<20 \mathrm{GeV}$ and $n_{\text {Tracks }}^{\mathrm{DV}}>2$; and

(3) DV Signal Region (DV SR): DVs passing the full selection.

For each muon background, a CR enriched with events of this background is defined by inverting the dedicated veto. A transfer factor is then determined as the ratio of the number of events passing the veto to the number of events rejected by it. As the probability of passing or failing the muon veto does not depend on the DV properties of the event, this transfer factor can be measured in the DV CR and applied in the DV SR in order to estimate the background contribution.

The following muon selections are used to define an orthogonal slicing of the dataset:

(i) Fake-muon $C R$ : full muon selection with the fakemuon veto inverted,

(ii) Heavy-flavor CR: full muon selection with the heavy-flavor veto inverted,

(iii) Cosmic-muon $C R$ : full muon selection with the cosmic-muon veto inverted, and

(iv) Muon SR: full muon selection.

The final signal regions used in this search are the intersections of the DV SR requirements and the Muon SR requirements.

\section{B. Transfer factor determination}

The DV CR is used to determine the transfer factors for each of the three muon backgrounds. The transfer factor $f_{i}$ is measured as

$$
f_{i}=\frac{N\left(\text { Preselected events; } \mathrm{DV} \in \mathrm{DV} \mathrm{CR} ; \mu \text { passes veto } i \text { and passes } d_{0} \text { selection } i\right)}{N\left(\text { Preselected events; DV } \in \mathrm{DV} \mathrm{CR} ; \mu \text { fails veto } i \text { and passes } d_{0} \text { selection } i\right)}
$$

where $i$ represents the fake-muon, heavy-flavor, and cosmic-muon backgrounds, and events in both the numerator and denominator are required to pass the other two vetoes that compose the full muon selection. Varying $d_{0}$ selections are applied for each background estimation and are described below.

The fake-muon and heavy-flavor transfer factors are determined in the $E_{\mathrm{T}}^{\text {miss }}$-triggered sample, but because a larger number of events with cosmic-ray muons are expected to be found in the muon-triggered sample, the corresponding transfer factor is determined in those events. Additionally, to minimize the impact of muons from heavy-flavor decays on the determination of the transfer factors for fake-muon and cosmic-muon backgrounds, those are determined using preselected muons with $5 \mathrm{~mm}<\left|d_{0}\right|<300 \mathrm{~mm}$. For similar reasons, the heavy-flavor transfer factor is measured using preselected muons with $1.5 \mathrm{~mm}<\left|d_{0}\right|<3.0 \mathrm{~mm}$. The transfer factor dependence on these requirements on muon $d_{0}$ are accounted for in a dedicated systematic uncertainty as discussed in Section VII.

Given these selections, both the numerator and denominator of the transfer factors are taken from regions pure in their respective backgrounds, using the inversion of dedicated vetos as well as event and muon properties characteristic of each background source. Table III reports the transfer factors extracted from the data in the DV CR, along with their uncertainties (discussed in Sec. VII).

Although the fake-muon and heavy-flavor transfer factors are measured in the $E_{\mathrm{T}}^{\text {miss }}$-triggered sample, they are also applied in the muon-triggered sample. The cosmic-ray transfer factor is similarly applied to the $E_{\mathrm{T}}^{\text {miss }}$-triggered sample. In order to ensure that the extrapolation between trigger streams does not lead to a bias, a separate measurement of the heavy-flavor transfer factor is made in the muon-triggered sample and compared with the nominal transfer factor. Agreement within uncertainties is observed. 
TABLE III. Transfer factors used to calculate background estimates, extracted from control regions.

\begin{tabular}{lc}
\hline \hline Background source $(i)$ & Transfer factor $\left(f_{i}\right)$ \\
\hline Cosmic-ray muons & $(4.0 \pm 0.2($ stat $) \pm 0.5($ syst $)) \times 10^{-3}$ \\
Fake muons & $(1.0 \pm 0.3($ stat $) \pm 0.5($ syst $)) \times 10^{-2}$ \\
Heavy-flavor muons & $(9.1 \pm 1.6($ stat $) \pm 4.0($ syst $)) \times 10^{-2}$ \\
\hline \hline
\end{tabular}

It is impossible to repeat this check with cosmic-ray and fake-muon transfer factors, because they are negligible backgrounds in the $E_{\mathrm{T}}^{\text {miss }}$-triggered and muon-triggered samples respectively. Furthermore, cosmic-ray muons are expected to exhibit the same characteristics regardless of the trigger used to collect the event.

\section{Background predictions}

Using the transfer factors determined from the data, the background in the final SRs of the analysis can be predicted for each source $i$ by multiplying the event yield observed in the corresponding muon $\mathrm{CR}, N_{i}^{\mathrm{CR}}$, in events with at least one DV passing the full selection (DV SR) by $f_{i}$. The total expected background $N_{\mathrm{B}}^{\mathrm{SR}}$ in each $\mathrm{SR}$ of the analysis is then given by

$$
N_{\mathrm{B}}^{\mathrm{SR}}=\sum_{i} N_{i}^{\mathrm{CR}} \times f_{i} .
$$

Similarly, the transfer factors are validated by comparing their predictions with the observed data in the DV VR to gain confidence that the method works. The VR is divided into two separate regions, one with two-track DVs and one with low-mass DVs with three tracks or more. The observed results of this validation are discussed in Sec. VIII.

Using the technique described above, the total predicted background yields are $0.43 \pm 0.16$ (stat) \pm 0.16 (syst) for the $E_{\mathrm{T}}^{\text {miss }}$ trigger SR and $1.88 \pm 0.20$ (stat) \pm 0.28 (syst) for the muon trigger SR. CR yields used in the background estimation are shown in Table IV along with predictions for the SRs. For the $E_{\mathrm{T}}^{\text {miss }}$ trigger SR, no events are observed in the cosmic-muon $\mathrm{CR}$, so fewer than 0.01 cosmic-ray muon events are expected in the SR at $68 \%$ confidence level (CL). This background component is therefore considered negligible in the presence of the other background contributions.

\section{UNCERTAINTIES}

Uncertainties affecting the estimated SR event yields for the various sources of background are assessed by using transfer factors extracted in subregions of the CRs probing different regions of DV properties, and taking the largest deviation from the nominal transfer factor measurement. The DV CR is divided into three subregions of events: those with no reconstructed DV and those with two-track DVs or three-track DVs that do not pass the material veto. Given the differing DV properties, the span of these transfer factors represents the overall uncertainty from residual correlations between DV and muon properties. Uncertainties are also assessed by measuring transfer factors with varying requirements on the muon $d_{0}$. For the fake-muon and cosmic-ray transfer factors, separate measurements are evaluated for muons with $5<\left|d_{0}\right|<$ $100 \mathrm{~mm}$ and $100<\left|d_{0}\right|<300 \mathrm{~mm}$. For the heavy-flavor transfer factor, transfer factors are evaluated for muons with $1.5<\left|d_{0}\right|<1.7 \mathrm{~mm}$ and $1.7<\left|d_{0}\right|<3 \mathrm{~mm}$. These impact parameter selections were designed to minimize the statistical uncertainties of the alternative transfer factor measurements. The spread of these transfer factors is measured to account for residual dependence of the transfer factor on the measured muon properties. The uncertainties from these two variations are added in quadrature. The uncertainties for the final SR background predictions quoted in Sec. VI are determined in this way.

A number of factors affect the uncertainty of the event yields predicted for signal scenarios, and this section outlines how their impact on the final event selection efficiencies $\epsilon_{\text {sel }}$ is determined.

The largest overall uncertainty is related to the dedicated LRT and secondary-vertex reconstruction. The radial distributions of secondary vertices from $K_{\mathrm{S}}^{0}$ decays are

TABLE IV. Control region yields $N_{i}^{\text {CR }}$ are shown for each major background for the $E_{\mathrm{T}}^{\text {miss }}$ trigger region and the muon trigger region, along with the result of scaling each background component by the appropriate transfer factor $f_{i}$. The total background yields $N_{\mathrm{B}}^{\mathrm{SR}}$ expected in each SR are also shown. The uncertainties shown are statistical and systematic, respectively. For the $E_{\mathrm{T}}^{\text {miss }}$ trigger region, the contribution from cosmic-ray muons is found to be negligible as described in the text.

\begin{tabular}{llrcc}
\hline \hline Selection & Background Source & $N_{i}^{\mathrm{CR}}$ & $N_{i}^{\mathrm{CR}} \times f_{i}$ & $N_{\mathrm{B}}^{\mathrm{SR}}$ \\
\hline \multirow{2}{*}{$E_{\mathrm{T}}^{\text {miss }}$ trigger } & Cosmic-ray muons & 0 & $\ldots$ & \\
& Fake muons & 16 & $0.16 \pm 0.04 \pm 0.09$ & \} $0.43 \pm 0.16 \pm 0.16$ \\
& Heavy-flavor muons & 3 & $0.27 \pm 0.16 \pm 0.13$ & \\
\multirow{2}{*}{ Muon trigger } & Cosmic-ray muons & 376 & $1.50 \pm 0.08 \pm 0.23$ & \\
& Fake muons & 1 & $0.01 \pm 0.01 \pm 0.01$ & \\
& Heavy-flavor muons & 4 & $0.36 \pm 0.18 \pm 0.17$ & \\
& & & & \\
\hline \hline
\end{tabular}


compared in data and Pythia multijet simulation, after normalizing the two distributions by the number of vertices at low radii, where tracking efficiencies are well understood. The mismodeling of the reconstruction efficiency in MC simulation at large track impact parameter and large vertex radius is estimated in this comparison. The largest observed difference is applied as a conservative per-track efficiency uncertainty of $\pm 10 \%$ which is then applied to the tracks associated with displaced vertices in the signal samples. The effect on final event counts with a fully selected DV is determined to be $15 \%$.

Additional uncertainty due to imperfect trigger efficiency is determined by comparing the $E_{\mathrm{T}}^{\text {miss }}$ trigger efficiency turn-on as function of cluster-based $E_{\mathrm{T}}^{\text {miss }}$ in $Z \rightarrow$ $\mu^{+} \mu^{-}$events in data and $\mathrm{MC}$ simulation. An $E_{\mathrm{T}}^{\text {miss }}$ dependent correction (between 0 and 10\%) is extracted and applied to the MC samples for events which have cluster-based $E_{\mathrm{T}}^{\text {miss }}$ between 180 and $220 \mathrm{GeV}$. The relative difference between the final SR event yields with and without this correction is taken as a systematic uncertainty, found to be $<0.2 \%$. The uncertainties in the scale and resolution for the cluster energies entering the $E_{\mathrm{T}}^{\text {miss }}$ calculation are assessed by studying the size of these uncertainties for fully reconstructed jets [75]. The same variations are also observed to have a small effect on the muon isolation efficiency. The combined effect is determined to be no more than $2.1 \%$, which is applied to all signal efficiencies.

For the efficiency of the MS-only muon trigger, the relative efficiency degradation as a function of $d_{0}$ with respect to prompt muons as determined in MC signal samples is taken as an uncertainty. The per-track reconstruction efficiency uncertainty extracted above from $K_{\mathrm{S}}^{0}$ decays is also applied for the ID part of the muon track reconstruction. The muon reconstruction and selection criteria efficiency are also determined in $\mathrm{MC}$, and the degradation as a function of $d_{0}$ with respect to prompt muons is taken as an uncertainty. The effects of these uncertainties are assumed to be uncorrelated. Their combined impact on the signal event yields in the SR is $10 \%-12 \%$. The uncertainty due to residual inefficiency in the MS-only muon trigger given prompt muon production is found to be $<0.2 \%$. In addition, since custom muon identification criteria are applied, differences between data and $\mathrm{MC}$ events are corrected for by comparing efficiencies for prompt muons in $Z \rightarrow \mu^{+} \mu^{-}$events in data and MC samples processed with the special reconstruction used for the analysis. The statistical uncertainties of these measured scale factors are propagated to the final SR event yield uncertainties for signal, resulting in an impact of $0.9 \%-4.0 \%$ on the total selection efficiency. This uncertainty is added in quadrature with standard prompt muon uncertainties due to mismeasurement and result in additional uncertainties below $1 \%$.

To assess the sensitivity to effects due to imperfect modeling of the pileup interactions in the signal samples, a
TABLE V. Summary of the impact of systematic uncertainties on the predicted yield for the signal scenarios with pair-produced long-lived top squarks. These uncertainties apply to both signal region selections used in the search unless otherwise specified.

\begin{tabular}{lc}
\hline \hline Source of Uncertainty & $\begin{array}{c}\text { Relative Impact Signal } \\
\text { Yield [\%] }\end{array}$ \\
\hline Total & $18-20$ \\
\hline Tracking and vertex reconstruction & 15 \\
Displaced muon efficiency & $10-12$ \\
Prompt muon efficiency & $(0.01-0.7) \oplus(0.9-4.0)$ \\
ISR modeling in MC simulation & $0.37-2.2$ \\
Pileup modeling & 2.1 \\
Hadronic energy scale and resolution & \\
$\quad$ (affecting $E_{\mathrm{T}}^{\text {miss }}$ ) & 1.7 \\
Integrated luminosity of dataset & $<0.2$ \\
Trigger efficiency & \\
\hline \hline
\end{tabular}

reweighting is applied to the MC signal samples to cover the uncertainty in the number of $p p$ interactions in the data. The largest relative difference in $\epsilon_{\text {sel }}$ is determined to be between $0.37 \%$ and $2.2 \%$. Another uncertainty stems from the modeling of initial-state QCD radiation (ISR), which affects the kinematics of the $\tilde{t} \tilde{t}$ system and thus the clusterbased $E_{\mathrm{T}}^{\mathrm{miss}}$ and the muon $p_{\mathrm{T}}$ distribution. To assess the sensitivity of $\epsilon_{\mathrm{sel}}$ to this, a reweighting of the $p_{\mathrm{T}}$ distribution of the $\tilde{t} \tilde{t}$ system in the MadGraph5_aMC@NLO events is applied to simulate that of PYTHIA 6.427. The resulting impact on $\epsilon_{\text {sel }}$ is $3 \%$. Finally, the uncertainty of $1.7 \%$ in the integrated luminosity of the dataset is included as that translates directly to an uncertainty in the expected yield of signal events in the SRs.

The impact of the systematic uncertainties affecting the signal yield in both signal regions is summarized in Table V.

\section{RESULTS}

No events are observed in the $E_{\mathrm{T}}^{\text {miss }}$ trigger SR where $0.43 \pm 0.16$ (stat) \pm 0.16 (syst) events are expected in the absence of signal, while a single event is observed in the muon trigger SR where $1.88 \pm 0.20$ (stat) \pm 0.28 (syst) events are expected. The observed event yields are in good agreement with the background-only expectations. The single event observed in the muon trigger SR contains a selected muon with $p_{\mathrm{T}}=103 \mathrm{GeV}$, and two selected DVs. One DV has $n_{\text {Tracks }}^{\mathrm{DV}}=3, m_{\mathrm{DV}}=23.5 \mathrm{GeV}$, and net electric charge of $+1 e$, while the other has $n_{\text {Tracks }}^{\mathrm{DV}}=3, m_{\mathrm{DV}}=$ $22.7 \mathrm{GeV}$, and a net electric charge of $-1 e$.

Figure 4 shows the distributions of the variables used for the final two DV-selection requirements in events with a muon passing the full muon selection. The track multiplicity $n_{\text {Tracks }}^{\text {DV }}$ is shown for all preselected displaced vertices. The invariant mass $m_{\mathrm{DV}}$ is shown for the highestmass preselected displaced vertex with at least three 

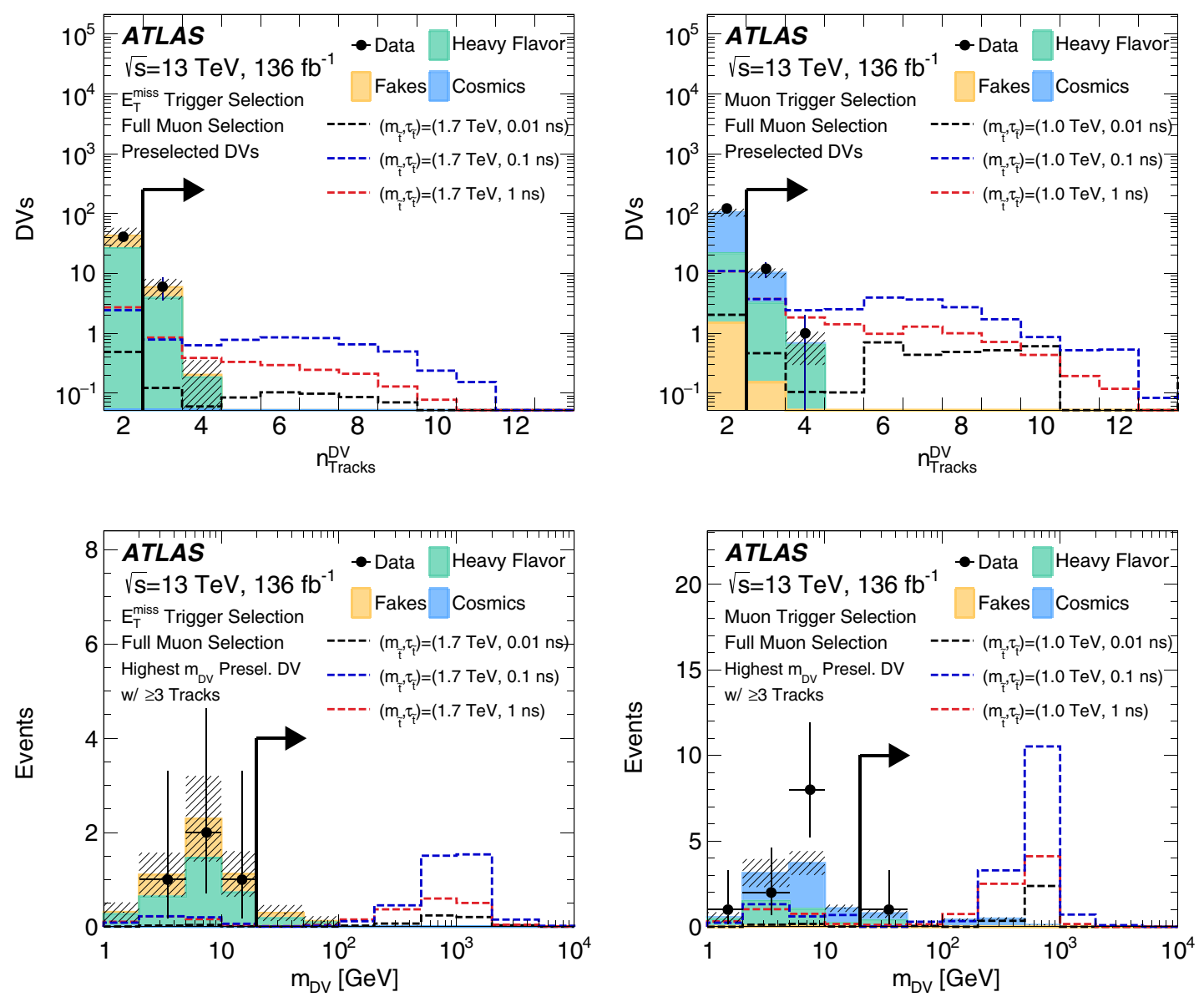

FIG. 4. Distributions of track multiplicity $n_{\text {Tracks }}^{\mathrm{DV}}$ (top) for preselected DVs, and invariant mass $m_{\mathrm{DV}}$ (bottom) for the highest-mass preselected DV with at least three associated tracks. This is shown for events with at least one muon passing the full selection, in the $E_{\mathrm{T}}^{\text {miss }}$-triggered (left) and muon-triggered (right) samples. Along with the data, shown with black markers, the stacked filled histograms represent the background estimates, and predictions for signal scenarios are overlaid with dashed lines. Signals with lifetimes of 0.01 , 0.1 , and $1 \mathrm{~ns}$ are shown in black, blue, and red, respectively. For the $E_{\mathrm{T}}^{\text {miss }}$-triggered (muon-triggered) selection, signal samples are shown with $m(\tilde{t})=1.7 \mathrm{TeV}(m(\tilde{t})=1.0 \mathrm{TeV})$. The errors include statistical and systematic uncertainties and are indicated by hatched bands. The DV full selection requirements, $n_{\text {Tracks }}^{\mathrm{DV}} \geq 3$ and $m_{\mathrm{DV}}>20 \mathrm{GeV}$ are visualized with a black arrow.

associated tracks. The individual background predictions are derived from muon CR distributions scaled by the transfer factors described in Sec. VI.

For all four distributions, there is good agreement between the number of vertices observed in data and the background prediction. The DV full selection requirements, $n_{\text {Tracks }}^{\text {DV }} \geq 3$ and $m_{\mathrm{DV}}>20 \mathrm{GeV}$ are visualized with a black arrow. For the $m_{\mathrm{DV}}$ distributions, inverting the requirement denoted by the black arrow gives the low-mass DV VR, showing good agreement between the expected background normalization and the observed number of events.

Signal and data event yields are shown as a function of progressively stricter requirements in Fig. 5 along with the cumulative selection efficiencies, including acceptance effects. For the signal model considered here, over $95 \%$ of events have cluster-based $E_{\mathrm{T}}^{\text {miss }}>180 \mathrm{GeV}$ and consequently the $E_{\mathrm{T}}^{\text {miss }}$ trigger $\mathrm{SR}$ has the larger cumulative selection efficiency. For signals with $\tau(\tilde{t})=0.1 \mathrm{~ns}$, the cumulative selection efficiency of the $E_{\mathrm{T}}^{\text {miss }}$ trigger $\mathrm{SR}$ is $35 \%$. Signals with $\tau(\tilde{t})=1$ ns have a cumulative selection efficiency of $15 \%$, due to the reduced efficiency for reconstructed displaced tracks and displaced vertices at large radii. Signals with $\tau(\tilde{t})=0.01 \mathrm{~ns}$ have cumulative selection efficiencies between $5 \%-6 \%$, because many $\tilde{t}$ squark decays occur within millimeters of the primary $p p$ vertex. The efficiency loss for signals with $\tau(\tilde{t})=0.01 \mathrm{~ns}$ is more pronounced due to the requirement of at least one muon with $\left|d_{0}\right|>2 \mathrm{~mm}$, and a DV which is at least $4 \mathrm{~mm}$ away from any $p p$ collision vertex in the event.

The final yields in the control, validation and signal regions are shown in Fig. 6. In the DV VRs, the data show good agreement with the background predictions within uncertainties, validating the assumptions made in the background estimation. In the SRs, the data show good agreement with the background predictions within uncertainties. 

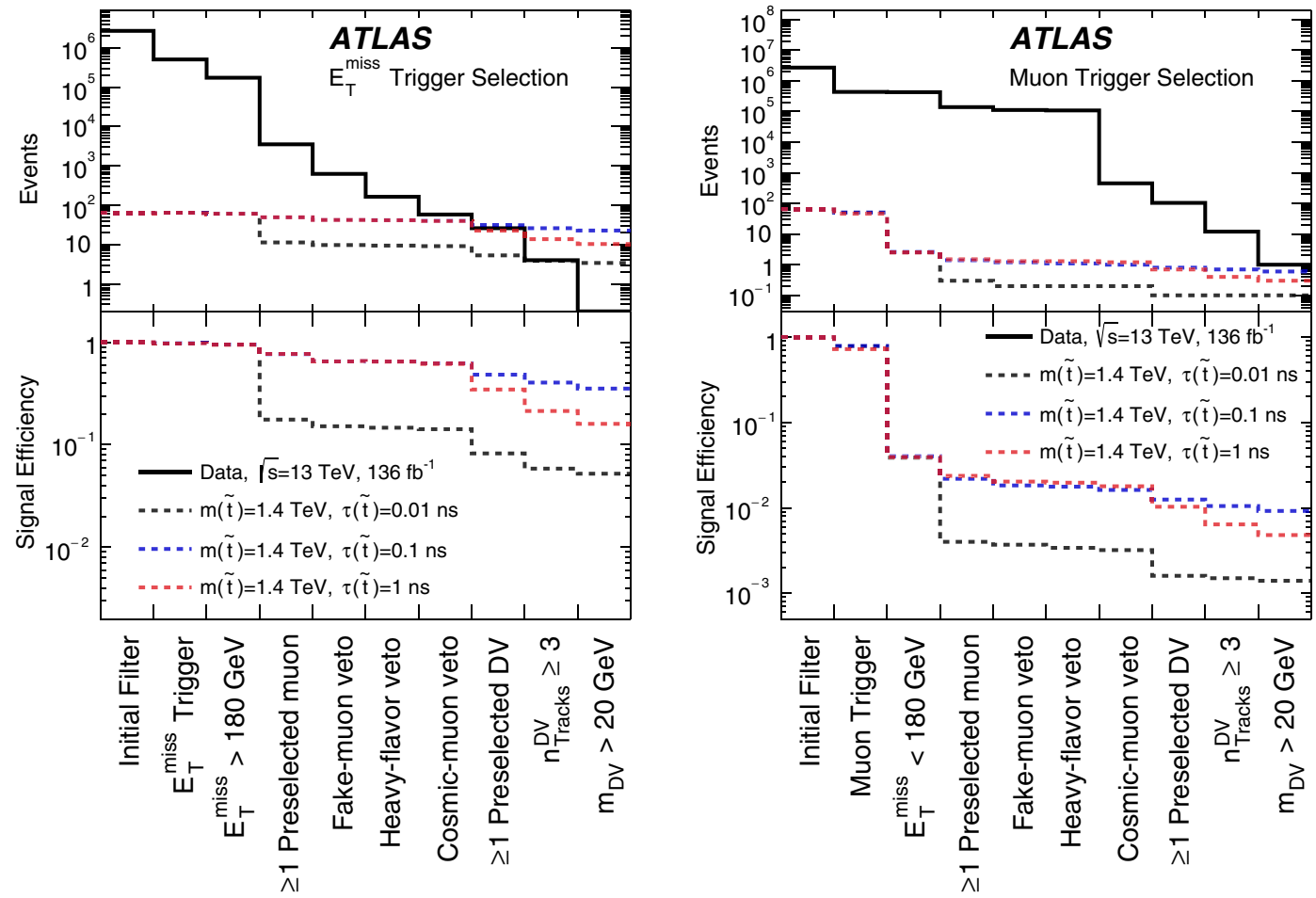

FIG. 5. Event yields are shown as a function of the SR requirements for data and three example signal models for the $E_{\mathrm{T}}^{\mathrm{miss}}$ trigger selection (left) and the muon trigger selection (right). The initial filter requirement includes selections on the data due to the raw-event filters. For the signals, no initial filter is applied and acceptance effects are included. The $E_{\mathrm{T}}^{\text {miss }}$ requirement label denotes requirements on the cluster-based $E_{\mathrm{T}}^{\text {miss }}$. Signals with lifetimes of $0.01,0.1$, and $1 \mathrm{~ns}$ are shown in black, blue, and red, respectively. Samples are shown for signals with $m(\tilde{t})=1.4 \mathrm{TeV}$.

The HistFitter package [76] was used for the calculation of the $95 \% \mathrm{CL}$ exclusion limits using the $\mathrm{CL}_{\mathrm{s}}$ prescription [77]. Figure 7 shows expected and observed 95\% CL exclusion limits on the mass of a long-lived $\tilde{t}$ squark as a function of its mean proper lifetime $\tau(\tilde{t})$. For each configuration of model parameters, the limit from the SR with the higher expected sensitivity is shown. The sensitivity to the shown region of parameter space comes entirely from the $E_{\mathrm{T}}^{\mathrm{miss}}$ trigger SR. Additionally, in Figure 8, 95\% CL upper limits on the production cross section of
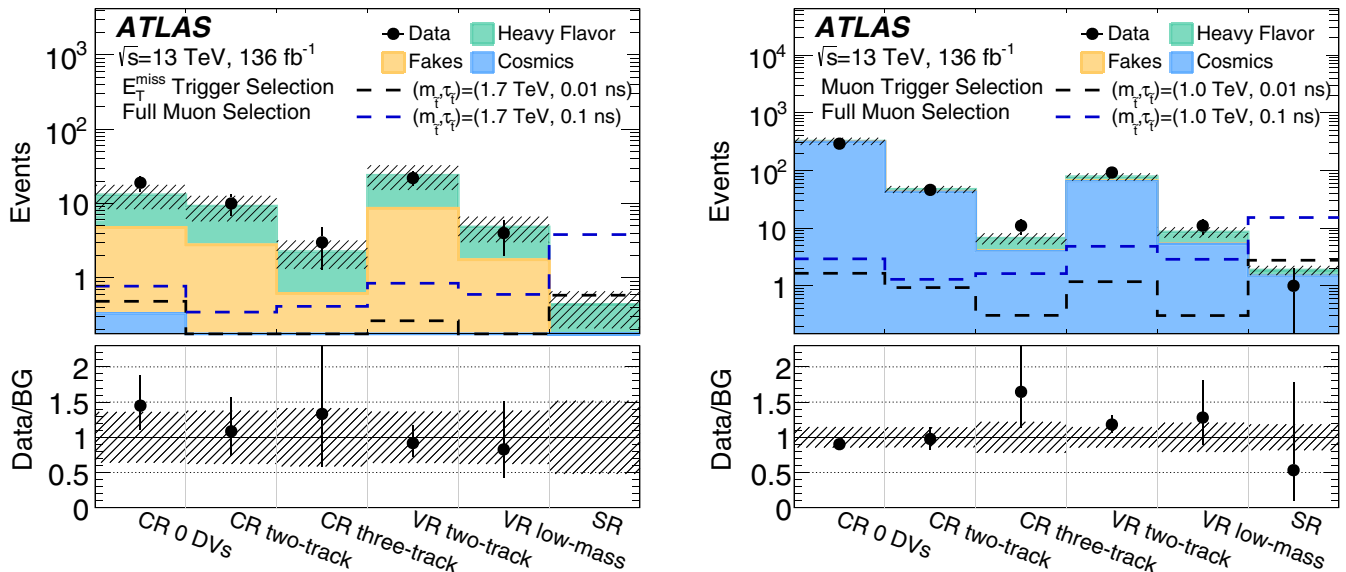

FIG. 6. The observed event yields in the control, validation and signal regions are shown for the $E_{\mathrm{T}}^{\mathrm{miss}}$ trigger (left) and muon trigger (right) selections, along with the predicted background yields. The bottom panel shows the ratio of observed events to the total background yields. Signals with lifetimes of $0.01 \mathrm{~ns}$ and $0.1 \mathrm{~ns}$ are shown in blue and red, respectively. For the $E_{\mathrm{T}}^{\text {miss }}$-triggered (muontriggered) selection, signal samples are shown with $m(\tilde{t})=1.7 \mathrm{TeV}(m(\tilde{t})=1.0 \mathrm{TeV})$. The errors represent the total uncertainty of the background predictions, including the statistical and systematic uncertainties added in quadrature. 


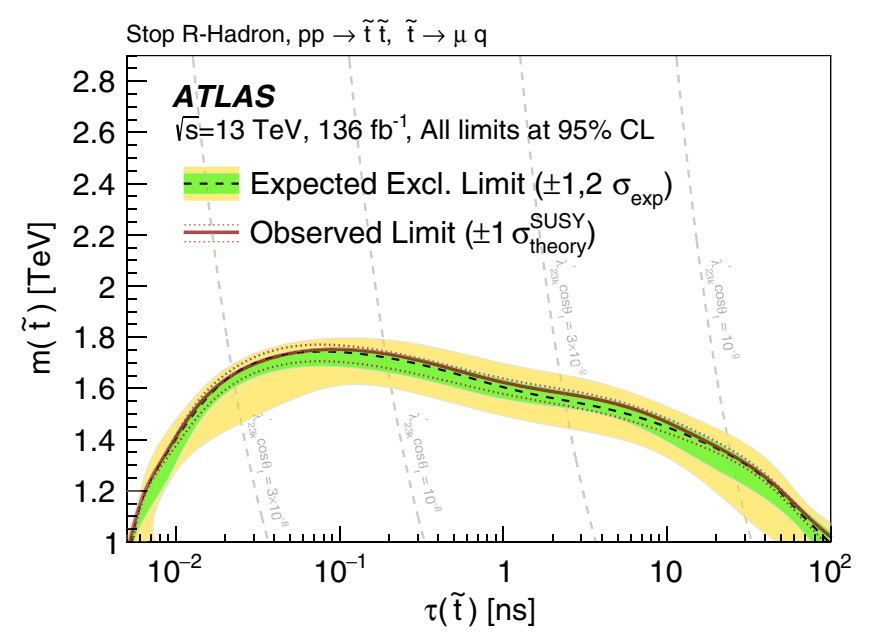

FIG. 7. Exclusion limits at $95 \% \mathrm{CL}$ on $m(\tilde{t})$ as a function of $\tau(\tilde{t})$ are shown along with contours showing fixed values of $\lambda_{23 k}^{\prime} \cos \theta_{t}$. Experimental and theoretical uncertainties are shown.

these signals are shown as a function of $m(\tilde{t})$ for various values of $\tau(\tilde{t})$. The limits show little variation as a function of $m(\tilde{t})$ owing to the fact that the signal acceptance and efficiency are relatively invariant in $m(\tilde{t})$ for the range considered here.

Contours showing fixed values of $\lambda_{23 k}^{\prime} \cos \theta_{t}$ are also shown in Fig. 7 where $\theta_{t}$ is the mixing angle between the left- and right-handed $\tilde{t}$ squarks. These contours are derived using the expression provided in Ref. [78] for a $\tilde{t}$ squark decay via a single $\lambda_{i j k}^{\prime}$ coupling:

$$
\tau(\tilde{t})=\frac{16 \pi}{m(\tilde{t})\left[\lambda_{23 k}^{\prime} \cos \theta_{t}\right]^{2}}
$$

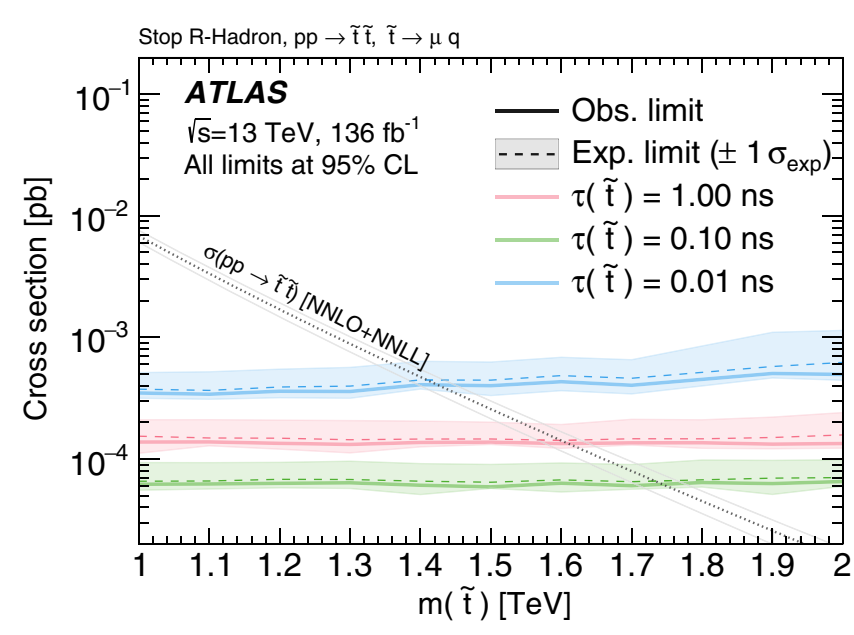

FIG. 8. Exclusion limits on the production cross section as a function of $m(\tilde{t})$ are shown for several values of $\tau(\tilde{t})$ along with the nominal signal production cross section and its theoretical uncertainty.
TABLE VI. The observed 95\% CL upper limits on the visible cross section $\left\langle\sigma_{\text {vis }}\right\rangle_{\text {obs }}^{95}$, and the observed $\left(S_{\text {obs }}^{95}\right)$ and expected $\left(S_{\exp }^{95}\right)$ limits on the number of signal events.

\begin{tabular}{lccc}
\hline \hline Signal Region & $S_{\text {obs }}^{95}$ & $S_{\text {exp }}^{95}$ & $\left\langle\sigma_{\text {vis }}\right\rangle_{\text {obs }}^{95}[\mathrm{fb}]$ \\
\hline$E_{\mathrm{T}}^{\text {miss }}$ trigger SR & 3.0 & $3.0_{-0.1}^{+1.1}$ & 0.023 \\
Muon trigger SR & 3.6 & $4.2_{-1.1}^{+1.9}$ & 0.027 \\
\hline \hline
\end{tabular}

For a mean proper lifetime of $\tau(\tilde{t})=0.1 \mathrm{~ns}$, masses below roughly $m(\tilde{t})=1.7 \mathrm{TeV}$ are excluded. For masses on the order of the DV mass requirement of $20 \mathrm{GeV}$ and below, sensitivity is expected to drastically decrease. For the wide range of mean proper lifetime values between 0.01 and $30 \mathrm{~ns}$, masses below $1.3 \mathrm{TeV}$ are excluded at 95\% CL. For $\tau(\tilde{t})=0.1 \mathrm{~ns}$, cross-section upper limits are set below $100 \mathrm{ab}$. For mean proper lifetimes between 0.01 and $100 \mathrm{ns,}$ these limits are the strictest to date on models with a metastable $\tilde{t}$ squark decaying via the $\lambda_{i j k}^{\prime}$ RPV coupling. For $m(\tilde{t})=1 \mathrm{TeV}$, values of $\lambda_{23 k}^{\prime} \cos \theta_{t}$ between roughly $10^{-7}$ and $10^{-9}$ are excluded at $95 \%$ CL.

Model-independent upper limits at 95\% CL on the number of BSM events in the signal region are also derived, assuming no significant contamination from alternate signal models in the control regions. Normalizing these limits by the integrated luminosity of the data sample, these numbers can be interpreted as upper limits on the visible BSM cross section, denoted by $\sigma_{\text {vis. }}$. It is defined as the product of signal acceptance, reconstruction efficiency, and production cross section. The results are given in Table VI.

\section{CONCLUSION}

A search for physics beyond the Standard Model giving rise to long-lived particle decays with muons is performed with the ATLAS experiment at the LHC using $136 \mathrm{fb}^{-1}$ of $p p$ collision data at $\sqrt{s}=13 \mathrm{TeV}$. Event selections are developed to efficiently reject backgrounds. The yields expected from background in the two orthogonal signal regions used in the analysis are extracted from control regions in the data and amount to $0.43 \pm 0.16$ (stat) \pm 0.16 (syst) in the $E_{\mathrm{T}}^{\text {miss }}$ trigger SR, and $1.88 \pm 0.20$ (stat) \pm 0.28 (syst) in the muon trigger SR. The data agree with the yields expected from the background-only hypothesis, with zero and one event passing the $E_{\mathrm{T}}^{\text {miss }}$ and muon trigger SR requirements, respectively.

The results are interpreted in a supersymmetric model with pair-produced top squarks $\tilde{t}$ decaying via small values of the $R$-parity-violating coupling $\lambda_{23 k}^{\prime}$ into a muon and a quark, giving the $\tilde{t}$ squark mean proper lifetimes $\tau(\tilde{t})$ in the ps to ns range. At $95 \%$ confidence level, $m(\tilde{t})$ values up to $1.7 \mathrm{TeV}$ for $\tau(\tilde{t})=0.1 \mathrm{~ns}$ are excluded, and the limit surpasses $1.3 \mathrm{TeV}$ for all lifetimes in the range from 0.01 to $30 \mathrm{~ns}$. Upper limits on the visible cross section 
for processes with the sought signature are $0.023 \mathrm{fb}$ and $0.027 \mathrm{fb}$ for the $E_{\mathrm{T}}^{\text {miss }}$ and muon trigger SRs, respectively.

\section{ACKNOWLEDGMENTS}

We thank CERN for the very successful operation of the LHC, as well as the support staff from our institutions without whom ATLAS could not be operated efficiently. We acknowledge the support of ANPCyT, Argentina; YerPhI, Armenia; ARC, Australia; BMWFW and FWF, Austria; ANAS, Azerbaijan; SSTC, Belarus; CNPq and FAPESP, Brazil; NSERC, NRC and CFI, Canada; CERN; CONICYT, Chile; CAS, MOST and NSFC, China; COLCIENCIAS, Colombia; MSMT CR, MPO CR and VSC CR, Czech Republic; DNRF and DNSRC, Denmark; IN2P3-CNRS and CEA-DRF/IRFU, France; SRNSFG, Georgia; BMBF, HGF and MPG, Germany; GSRT, Greece; RGC and Hong Kong SAR, China; ISF and Benoziyo Center, Israel; INFN, Italy; MEXT and JSPS, Japan; CNRST, Morocco; NWO, Netherlands; RCN, Norway; MNiSW and NCN, Poland; FCT, Portugal; MNE/IFA, Romania; MES of Russia and NRC KI, Russia Federation; JINR; MESTD, Serbia; MSSR, Slovakia; ARRS and MIZŠ, Slovenia; DST/NRF, South Africa; MINECO, Spain; SRC and Wallenberg Foundation,
Sweden; SERI, SNSF and Cantons of Bern and Geneva, Switzerland; MOST, Taiwan; TAEK, Turkey; STFC, United Kingdom; DOE and NSF, United States of America. In addition, individual groups and members have received support from BCKDF, CANARIE, Compute Canada and CRC, Canada; ERC, ERDF, Horizon 2020, Marie Skłodowska-Curie Actions and COST, European Union; Investissements d'Avenir Labex, Investissements d'Avenir Idex and ANR, France; DFG and AvH Foundation, Germany; Herakleitos, Thales and Aristeia programmes co-financed by EU-ESF and the Greek NSRF, Greece; BSF-NSF and GIF, Israel; CERCA Programme Generalitat de Catalunya and PROMETEO Programme Generalitat Valenciana, Spain; Göran Gustafssons Stiftelse, Sweden; The Royal Society and Leverhulme Trust, United Kingdom. The crucial computing support from all WLCG partners is acknowledged gratefully, in particular from CERN, the ATLAS Tier-1 facilities at TRIUMF (Canada), NDGF (Denmark, Norway, Sweden), CCIN2P3 (France), KIT/GridKA (Germany), INFN-CNAF (Italy), NL-T1 (Netherlands), PIC (Spain), ASGC (Taiwan), RAL (UK) and BNL (USA), the Tier-2 facilities worldwide and large non-WLCG resource providers. Major contributors of computing resources are listed in Ref. [79].
[1] R. Barbieri and G. Giudice, Upper bounds on supersymmetric particle masses, Nucl. Phys. B306, 63 (1988).

[2] B. de Carlos and J. Casas, One-loop analysis of the electroweak breaking in supersymmetric models and the fine-tuning problem, Phys. Lett. B 309, 320 (1993).

[3] G. Bertone, D. Hooper, and J. Silk, Particle dark matter: Evidence, candidates and constraints, Phys. Rep. 405, 279 (2005).

[4] M. Fairbairn, A. C. Kraan, D. A. Milstead, T. Sjöstrand, P. Skands, and T. Sloan, Stable massive particles at colliders, Phys. Rep. 438, 1 (2007).

[5] L. Lee, C. Ohm, A. Soffer, and T.-T. Yu, Collider searches for long-lived particles beyond the Standard Model, Prog. Part. Nucl. Phys. 106, 210 (2019).

[6] Y. Golfand and E. Likhtman, Extension of the algebra of Poincare group generators and violation of $\mathrm{P}$ invariance, PismaZh. Eksp. Teor. Fiz. 13, 452 (1971) [JETP Lett. 13, 323 (1971)].

[7] D. Volkov and V. Akulov, Is the neutrino a goldstone particle?, Phys. Lett. 46B, 109 (1973).

[8] J. Wess and B. Zumino, Supergauge transformations in four dimensions, Nucl. Phys. B70, 39 (1974).

[9] J. Wess and B. Zumino, Supergauge invariant extension of quantum electrodynamics, Nucl. Phys. B78, 1 (1974).

[10] S. Ferrara and B. Zumino, Supergauge invariant Yang-Mills theories, Nucl. Phys. B79, 413 (1974).
[11] A. Salam and J. Strathdee, Super-symmetry and nonAbelian gauges, Phys. Lett. 51B, 353 (1974).

[12] N. Sakai, Naturalness in supersymmetric GUTS, Z. Phys. C 11, 153 (1981).

[13] S. Dimopoulos, S. Raby, and F. Wilczek, Supersymmetry and the scale of unification, Phys. Rev. D 24, 1681 (1981).

[14] L. E. Ibáñez and G. G. Ross, Low-energy predictions in supersymmetric grand unified theories, Phys. Lett. B 105, 439 (1981).

[15] S. Dimopoulos and H. Georgi, Softly broken supersymmetry and SU(5), Nucl. Phys. B193, 150 (1981).

[16] K. Inoue, A. Kakuto, H. Komatsu, and S. Takeshita, Aspects of grand unified models with softly broken supersymmetry, Prog. Theor. Phys. 68, 927 (1982); Erratum, Prog. Theor. Phys. 70, 330 (1983).

[17] J. R. Ellis and S. Rudaz, Search for supersymmetry in toponium decays, Phys. Lett. 128B, 248 (1983).

[18] R. Barbier et al., R-parity violating supersymmetry, Phys. Rep. 420, 1 (2005).

[19] G. 't Hooft, Computation of the quantum effects due to a four-dimensional pseudoparticle, Phys. Rev. D 14, 3432 (1976).

[20] V. A. Kuzmin, V. A. Rubakov, and M. E. Shaposhnikov, On anomalous electroweak baryon-number non-conservation in the early universe, Phys. Lett. 155B, 36 (1985). 
[21] P. Fayet, Supersymmetry and Weak, Electromagnetic and strong interactions, Phys. Lett. 64B, 159 (1976).

[22] P. Fayet, Spontaneously broken supersymmetric theories of weak, electromagnetic and strong interactions, Phys. Lett. 69B, 489 (1977).

[23] H. Goldberg, Constraint on the Photino Mass from Cosmology, Phys. Rev. Lett. 50, 1419 (1983); Erratum, Phys. Rev. Lett. 103, 099905 (2009).

[24] J. Ellis, J. Hagelin, D. V. Nanopoulos, K. A. Olive, and M. Srednicki, Supersymmetric relics from the big bang, Nucl. Phys. B238, 453 (1984).

[25] C. Friberg, E. Norrbin, and T. Sjöstrand, QCD aspects of leptoquark production at HERA, Phys. Lett. B 403, 329 (1997).

[26] M. J. Strassler and K. M. Zurek, Discovering the Higgs through highly-displaced vertices, Phys. Lett. B 661, 263 (2008).

[27] Y. Cui and B. Shuve, Probing baryogenesis with displaced vertices at the LHC, J. High Energy Phys. 02 (2015) 049.

[28] T. Asaka and M. Shaposhnikov, The nuMSM, dark matter and baryon asymmetry of the universe, Phys. Lett. B 620, 17 (2005).

[29] B. C. Allanach, M. A. Bernhardt, H. K. Dreiner, C. H. Kom, and P. Richardson, Mass spectrum in R-parity violating minimal supergravity and benchmark points, Phys. Rev. D 75, 035002 (2007).

[30] ATLAS Collaboration, Search for B-L R-parity-violating top squarks in $\sqrt{s}=13 \mathrm{TeV}$ pp collisions with the ATLAS experiment, Phys. Rev. D 97, 032003 (2018).

[31] CMS Collaboration, Search for pair production of secondgeneration leptoquarks at $\sqrt{s}=13 \mathrm{TeV}$, Phys. Rev. D 99, 032014 (2019).

[32] CMS Collaboration, Search for new long-lived particles at $\sqrt{s}=13$ TeV, Phys. Lett. B 780, 432 (2018).

[33] CMS Collaboration, Search for Displaced Supersymmetry in Events with an Electron and a Muon with Large Impact Parameters, Phys. Rev. Lett. 114, 061801 (2015).

[34] CMS Collaboration, Search for long-lived particles decaying into displaced jets in proton-proton collisions at $\sqrt{s}=13 \mathrm{TeV}$, Phys. Rev. D 99, 032011 (2019).

[35] ATLAS Collaboration, Search for displaced vertices arising from decays of new heavy particles in $7 \mathrm{TeV}$ pp collisions at ATLAS, Phys. Lett. B 707, 478 (2012).

[36] ATLAS Collaboration, Search for long-lived, heavy particles in final states with a muon and multi-track displaced vertex in proton-proton collisions at $\sqrt{s}=7 \mathrm{TeV}$ with the ATLAS detector, Phys. Lett. B 719, 280 (2013).

[37] ATLAS Collaboration, The ATLAS experiment at the CERN large hadron collider, J. Instrum. 3, S08003 (2008).

[38] ATLAS Collaboration, ATLAS insertable B-layer technical design report, CERN Report No. ATLAS-TDR-19, 2010, https://cds.cern.ch/record/1291633; CERN Report No. ATLAS-TDR-19-ADD-1, 2012, https://cds.cern.ch/record/ 1451888.

[39] B. Abbott et al., Production and integration of the ATLAS insertable B-layer, J. Instrum. 13, T05008 (2018).

[40] ATLAS Collaboration, Performance of the ATLAS trigger system in 2015, Eur. Phys. J. C 77, 317 (2017).

[41] ATLAS Collaboration, ATLAS data quality operations and performance for 2015-2018 data-taking, J. Instrum. 15, P04003 (2020).
[42] ATLAS Collaboration, Luminosity determination in $p p$ collisions at $\sqrt{s}=13 \mathrm{TeV}$ using the ATLAS detector at the LHC, 2019, http://cds.cern.ch/record/2677054.

[43] G. Avoni et al., The new LUCID-2 detector for luminosity measurement and monitoring in ATLAS, J. Instrum. 13, P07017 (2018).

[44] J. Alwall, R. Frederix, S. Frixione, V. Hirschi, F. Maltoni, O. Mattelaer, H.-S. Shao, T. StelzerP. Torrielli, and M. Zaro, The automated computation of tree-level and next-to-leading order differential cross sections, and their matching to parton shower simulations, J. High Energy Phys. 07 (2014) 079.

[45] T. Sjöstrand, S. Ask, J. R. Christiansen, R. Corke, N. Desai, P. Ilten, S. Mrenna, S. Prestel, C. O. Rasmussen, and P.Z. Skands, An introduction to PYTHiA 8.2, Comput. Phys. Commun. 191, 159 (2015).

[46] ATLAS Collaboration, ATLAS PYTHIA 8 tunes to $7 \mathrm{TeV}$ data, CERN Report No. ATL-PHYS-PUB-2014-021, 2014, https://cds.cern.ch/record/1966419.

[47] R. D. Ball et al., Parton distributions with LHC data, Nucl. Phys. B867, 244 (2013).

[48] G. R. Farrar and P. Fayet, Phenomenology of the production, decay, and detection of new hadronic states associated with supersymmetry, Phys. Lett. 76B, 575 (1978).

[49] ATLAS Collaboration, The ATLAS simulation infrastructure, Eur. Phys. J. C 70, 823 (2010).

[50] S. Agostinelli et al., GEANT4-A simulation toolkit, Nucl. Instrum. Methods Phys. Res., Sect. A 506, 250 (2003).

[51] A.C. Kraan, Interactions of heavy stable hadronizing particles, Eur. Phys. J. C 37, 91 (2004).

[52] R. Mackeprang and A. Rizzi, Interactions of coloured heavy stable particles in matter, Eur. Phys. J. C 50, 353 (2007).

[53] R. Mackeprang and D. A. Milstead, An updated description of heavy-hadron interactions in Geant-4, Eur. Phys. J. C 66, 493 (2010).

[54] ATLAS Collaboration, Generation and simulation of $R$ hadrons in the ATLAS experiment, CERN Report No. ATLPHYS-PUB-2019-019, 2019, https://cds.cern.ch/record/ 2676309.

[55] W. Beenakker, C. Borschensky, M. Krämer, A. Kulesza, and E. Laenen, NNLL-fast: Predictions for coloured supersymmetric particle production at the LHC with threshold and Coulomb resummation, J. High Energy Phys. 12 (2016) 133.

[56] W. Beenakker, C. Borschensky, M. Krämer, A. Kulesza, E. Laenen, V. Theeuwes, and S. Thewes, NNLL resummation for Squark-Antisquark and Gluino-Pair production at the LHC, J. High Energy Phys. 12 (2014) 023.

[57] W. Beenakker, T. Janssen, S. Lepoeter, M. Krämer, A. Kulesza, E. Laenen, I. Niessen, S. Thewes, and T. Van Daal, Towards NNLL resummation: Hard matching coefficients for squark and gluino hadroproduction, J. High Energy Phys. 10 (2013) 120.

[58] W. Beenakker, S. Brensing, M. Krämer, A. Kulesza, E. Laenen, and I. Niessen, NNLL resummation for squarkantisquark pair production at the LHC, J. High Energy Phys. 01 (2012) 076.

[59] W. Beenakker, S. Brensing, M. Krämer, A. Kulesza, E. Laenen, and I. Niessen, Soft-gluon resummation for squark 
and gluino hadroproduction, J. High Energy Phys. 12 (2009) 041.

[60] A. Kulesza and L. Motyka, Soft gluon resummation for the production of gluino-gluino and squark-antisquark pairs at the LHC, Phys. Rev. D 80, 095004 (2009).

[61] A. Kulesza and L. Motyka, Threshold Resummation for Squark-Antisquark and Gluino-Pair Production at the LHC, Phys. Rev. Lett. 102, 111802 (2009).

[62] W. Beenakker, R. Höpker, M. Spira, and P. Zerwas, Squark and gluino production at hadron colliders, Nucl. Phys. B492, 51 (1997).

[63] J. Butterworth et al., PDF4LHC recommendations for LHC Run II, J. Phys. G 43, 023001 (2016).

[64] P. Nason, A new method for combining NLO QCD with shower Monte Carlo algorithms, J. High Energy Phys. 11 (2004) 040.

[65] S. Frixione, P. Nason, and C. Oleari, Matching NLO QCD computations with parton shower simulations: The POWHEG method, J. High Energy Phys. 11 (2007) 070.

[66] S. Alioli, P. Nason, C. Oleari, and E. Re, A general framework for implementing NLO calculations in shower Monte Carlo programs: the POWHEG BOX, J. High Energy Phys. 06 (2010) 043.

[67] S. Alioli, P. Nason, C. Oleari, and E. Re, NLO vector-boson production matched with shower in POWHEG, J. High Energy Phys. 07 (2008) 060.

[68] T. Sjöstrand, S. Mrenna, and P.Z. Skands, A brief introduction to PYTHIA 8.1, Comput. Phys. Commun. 178, 852 (2008).

[69] ATLAS Collaboration, The PYTHIA 8 A3 tune description of ATLAS minimum bias and inelastic measurements incorporating the Donnachie-Landshoff diffractive model, CERN Report No. ATL-PHYS-PUB-2016-017, 2016, https://cds.cern.ch/record/2206965.
[70] ATLAS Collaboration, Vertex reconstruction performance of the ATLAS detector at $\sqrt{s}=13 \mathrm{TeV}$, CERN Report No. ATL-PHYS-PUB-2015-026, 2015, https://cds.cern.ch/ record/2037717.

[71] ATLAS Collaboration, Performance of the reconstruction of large impact parameter tracks in the inner detector of ATLAS, CERN Report No. ATL-PHYS-PUB-2017-014, 2017, https://cds.cern.ch/record/2275635.

[72] ATLAS Collaboration, Muon reconstruction performance of the ATLAS detector in proton-proton collision data at $\sqrt{s}=13 \mathrm{TeV}$, Eur. Phys. J. C 76, 292 (2016).

[73] ATLAS Collaboration, Performance of vertex reconstruction algorithms for detection of new long-lived particle decays within the ATLAS inner detector, CERN Report No. ATL-PHYS-PUB-2019-013, 2019, https://cds .cern.ch/record/2669425.

[74] ATLAS Collaboration, Topological cell clustering in the ATLAS calorimeters and its performance in LHC Run 1, Eur. Phys. J. C 77, 490 (2017).

[75] ATLAS Collaboration, Jet energy scale measurements and their systematic uncertainties in proton-proton collisions at $\sqrt{s}=13 \mathrm{TeV}$ with the ATLAS detector, Phys. Rev. D 96, 072002 (2017).

[76] M. Baak, G. J. Besjes, D. Côté, A. Koutsman, J. Lorenz, and D. Short, HistFitter software framework for statistical data analysis, Eur. Phys. J. C 75, 153 (2015).

[77] A.L. Read, Presentation of search results: The $C L_{S}$ technique, J. Phys. G 28, 2693 (2002).

[78] T. Kon, T. Kobayashi, and S. Kitamura, Signatures of scalar top with R-parity breaking coupling at HERA, Phys. Lett. B 333, 263 (1994).

[79] ATLAS Collaboration, ATLAS computing acknowledgements, CERN Report No. ATL-GEN-PUB-2016-002, https://cds.cern.ch/record/2202407.

G. Aad, ${ }^{102}$ B. Abbott, ${ }^{129}$ D. C. Abbott, ${ }^{103}$ A. Abed Abud,${ }^{71 a, 71 b}$ K. Abeling, ${ }^{53}$ D. K. Abhayasinghe, ${ }^{94}$ S. H. Abidi, ${ }^{167}$ O. S. AbouZeid, ${ }^{40}$ N. L. Abraham, ${ }^{156}$ H. Abramowicz, ${ }^{161}$ H. Abreu, ${ }^{160}$ Y. Abulaiti, ${ }^{6}$ B. S. Acharya, ${ }^{67 a, 67 b, b}$ B. Achkar, ${ }^{53}$ S. Adachi, ${ }^{163}$ L. Adam, ${ }^{100}$ C. Adam Bourdarios, ${ }^{5}$ L. Adamczyk, ${ }^{84 a}$ L. Adamek, ${ }^{167}$ J. Adelman, ${ }^{121}$ M. Adersberger, ${ }^{14}$ A. Adiguzel, ${ }^{12 \mathrm{c}} \mathrm{S}$. Adorni, ${ }^{54}$ T. Adye, ${ }^{144}$ A. A. Affolder, ${ }^{146}$ Y. Afik, ${ }^{160}$ C. Agapopoulou, ${ }^{65}$ M. N. Agaras,${ }^{38}$ A. Aggarwal, ${ }^{119}$ C. Agheorghiesei, ${ }^{27 \mathrm{c}}$ J. A. Aguilar-Saavedra, ${ }^{140 \mathrm{f}, 140 \mathrm{a}, \mathrm{c}}$ F. Ahmadov, ${ }^{80}$ W. S. Ahmed, ${ }^{104}$ X. Ai, ${ }^{18}$ G. Aielli, ${ }^{74 a, 74 \mathrm{~b}}$ S. Akatsuka, ${ }^{86}$ T. P. A. Åkesson, ${ }^{97}$ E. Akilli, ${ }^{54}$ A. V. Akimov, ${ }^{111}$ K. Al Khoury, ${ }^{65}$ G. L. Alberghi, ${ }^{23 b, 23 a}$ J. Albert, ${ }^{176}$

M. J. Alconada Verzini, ${ }^{161}$ S. Alderweireldt ${ }^{36}$ M. Aleksa, ${ }^{36}$ I. N. Aleksandrov,${ }^{80}$ C. Alexa, ${ }^{27 b}$ D. Alexandre,${ }^{19}$ T. Alexopoulos, ${ }^{10}$ A. Alfonsi, ${ }^{120}$ F. Alfonsi, ${ }^{23 b, 23 a}$ M. Alhroob, ${ }^{129}$ B. Ali, ${ }^{142}$ G. Alimonti, ${ }^{69 a}$ J. Alison, ${ }^{37}$ S. P. Alkire, ${ }^{148}$ C. Allaire, ${ }^{65}$ B. M. M. Allbrooke, ${ }^{156}$ B. W. Allen, ${ }^{132}$ P. P. Allport, ${ }^{21}$ A. Aloisio $,{ }^{70 a}, 70 b$ A. Alonso, ${ }^{40}$ F. Alonso, ${ }^{89}$ C. Alpigiani, ${ }^{148}$ A. A. Alshehri, ${ }^{57}$ M. Alvarez Estevez, ${ }^{99}$ D. Álvarez Piqueras, ${ }^{174}$ M. G. Alviggi, ${ }^{70 a, 70 b}$

Y. Amaral Coutinho, ${ }^{81 b}$ A. Ambler, ${ }^{104}$ L. Ambroz, ${ }^{135}$ C. Amelung, ${ }^{26}$ D. Amidei, ${ }^{106}$ S. P. Amor Dos Santos, ${ }^{140 a}$ S. Amoroso, ${ }^{46}$ C. S. Amrouche, ${ }^{54}$ F. An,${ }^{79}$ C. Anastopoulos, ${ }^{149}$ N. Andari, ${ }^{145}$ T. Andeen, ${ }^{11}$ C. F. Anders, ${ }^{61 b}$ J. K. Anders,${ }^{20}$ A. Andreazza,${ }^{6 a, 69 b}$ V. Andrei ${ }^{61 a}$ C. R. Anelli, ${ }^{176}$ S. Angelidakis, ${ }^{38}$ A. Angerami, ${ }^{39}$ A. V. Anisenkov, ${ }^{122 b, 122 a}$ A. Annovi, ${ }^{72 a}$ C. Antel ${ }^{61 \mathrm{a}}$ M. T. Anthony, ${ }^{149}$ M. Antonelli, ${ }^{51}$ D. J. A. Antrim, ${ }^{171}$ F. Anulli, ${ }^{73 a}$ M. Aoki, ${ }^{82}$ J. A. Aparisi Pozo, ${ }^{174}$ L. Aperio Bella, ${ }^{15 a}$ G. Arabidze, ${ }^{107}$ J. P. Araque, ${ }^{140 \mathrm{a}}$ V. Araujo Ferraz ${ }^{81 \mathrm{~b}}$ R. Araujo Pereira, ${ }^{81 \mathrm{~b}}$ C. Arcangeletti, ${ }^{51}$ A. T. H. Arce, ${ }^{49}$ F. A. Arduh, ${ }^{89}$ J-F. Arguin, ${ }^{110}$ S. Argyropoulos, ${ }^{78}$ J.-H. Arling, ${ }^{46}$ A. J. Armbruster, ${ }^{36}$ A. Armstrong, ${ }^{171}$ O. Arnaez, ${ }^{167}$ H. Arnold, ${ }^{120}$ Z. P. Arrubarrena Tame, ${ }^{114}$ A. Artamonov, ${ }^{124, a}$ G. Artoni, ${ }^{135}$ S. Artz, ${ }^{100}$ S. Asai, ${ }^{163}$ N. Asbah,${ }^{59}$ E. M. Asimakopoulou, ${ }^{172}$ L. Asquith, ${ }^{156}$ J. Assahsah, ${ }^{35 \mathrm{~d}}$ K. Assamagan, ${ }^{29}$ R. Astalos, ${ }^{28 a}$ R. J. Atkin, ${ }^{33 a}$ M. Atkinson, ${ }^{173}$ 
N. B. Atlay, ${ }^{19}$ H. Atmani, ${ }^{65}$ K. Augsten, ${ }^{142}$ G. Avolio, ${ }^{36}$ R. Avramidou, ${ }^{60 a}$ M. K. Ayoub, ${ }^{15 a}$ A. M. Azoulay, ${ }^{168 b}$ G. Azuelos, ${ }^{110, \mathrm{~d}} \mathrm{H}$. Bachacou, ${ }^{145} \mathrm{~K}$. Bachas, ${ }^{68 \mathrm{a}, 68 \mathrm{~b}} \mathrm{M}$. Backes, ${ }^{135} \mathrm{~F}$. Backman, ${ }^{45 \mathrm{a}, 45 \mathrm{~b}}$ P. Bagnaia, ${ }^{73 \mathrm{a}, 73 \mathrm{~b}} \mathrm{M}$. Bahmani, ${ }^{85}$ H. Bahrasemani, ${ }^{152}$ A. J. Bailey, ${ }^{174}$ V. R. Bailey, ${ }^{173}$ J. T. Baines, ${ }^{144}$ M. Bajic, ${ }^{40}$ C. Bakalis, ${ }^{10}$ O. K. Baker, ${ }^{183}$ P. J. Bakker, ${ }^{120}$ D. Bakshi Gupta, ${ }^{8}$ S. Balaji, ${ }^{157}$ E. M. Baldin, ${ }^{122 b, 122 a}$ P. Balek, ${ }^{180}$ F. Balli, ${ }^{145}$ W. K. Balunas, ${ }^{135}$ J. Balz, ${ }^{100}$ E. Banas, ${ }^{85}$

A. Bandyopadhyay, ${ }^{24}$ Sw. Banerjee, ${ }^{181, e}$ A. A. E. Bannoura, ${ }^{182}$ L. Barak, ${ }^{161}$ W. M. Barbe,${ }^{38}$ E. L. Barberio, ${ }^{105}$ D. Barberis, ${ }^{55 b, 55 a}$ M. Barbero, ${ }^{102}$ G. Barbour, ${ }^{95}$ T. Barillari, ${ }^{115}$ M-S. Barisits, ${ }^{36}$ J. Barkeloo, ${ }^{132}$ T. Barklow, ${ }^{153}$ R. Barnea, ${ }^{160}$ S. L. Barnes, ${ }^{60 \mathrm{c}}$ B. M. Barnett, ${ }^{144}$ R. M. Barnett, ${ }^{18}$ Z. Barnovska-Blenessy, ${ }^{60 \mathrm{a}}$ A. Baroncelli, ${ }^{60 \mathrm{a}}$ G. Barone, ${ }^{29}$ A. J. Barr, ${ }^{135}$ L. Barranco Navarro, ${ }^{45 a, 45 b}$ F. Barreiro, ${ }^{99}$ J. Barreiro Guimarães da Costa, ${ }^{15 a}$ S. Barsov, ${ }^{138}$ R. Bartoldus, ${ }^{153}$ G. Bartolini, ${ }^{102}$ A. E. Barton, ${ }^{90}$ P. Bartos, ${ }^{28 a}$ A. Basalaev, ${ }^{46}$ A. Bassalat, ${ }^{65, f}$ M. J. Basso, ${ }^{167}$ R. L. Bates,${ }^{57}$ S. Batlamous, ${ }^{35 e}$ J. R. Batley, ${ }^{32}$ B. Batool, ${ }^{151}$ M. Battaglia, ${ }^{146}$ M. Bauce, ${ }^{73 a, 73 b}$ F. Bauer, ${ }^{145}$ K. T. Bauer, ${ }^{171}$ H. S. Bawa, ${ }^{31, g}$ J. B. Beacham, ${ }^{49}$ T. Beau, ${ }^{136}$ P. H. Beauchemin, ${ }^{170}$ F. Becherer, ${ }^{52}$ P. Bechtle, ${ }^{24}$ H. C. Beck, ${ }^{53}$ H. P. Beck,${ }^{20, \mathrm{~h}}$ K. Becker, ${ }^{52}$ M. Becker, ${ }^{100}$ C. Becot, ${ }^{46}$

A. Beddall, ${ }^{12 \mathrm{~d}}$ A. J. Beddall, ${ }^{12 \mathrm{a}}$ V. A. Bednyakov, ${ }^{80}$ M. Bedognetti, ${ }^{120}$ C. P. Bee, ${ }^{155}$ T. A. Beermann, ${ }^{77}$ M. Begalli, ${ }^{81 \mathrm{~b}}$ M. Begel, ${ }^{29}$ A. Behera, ${ }^{155}$ J. K. Behr,${ }^{46}$ F. Beisiegel,${ }^{24}$ A. S. Bell, ${ }^{95}$ G. Bella, ${ }^{161}$ L. Bellagamba, ${ }^{23 b}$ A. Bellerive, ${ }^{34}$ P. Bellos, ${ }^{9}$ K. Beloborodov, ${ }^{122 \mathrm{~b}, 122 \mathrm{a}}$ K. Belotskiy, ${ }^{112}$ N. L. Belyaev, ${ }^{112}$ D. Benchekroun, ${ }^{35 \mathrm{a}}$ N. Benekos,${ }^{10}$ Y. Benhammou, ${ }^{161}$

D. P. Benjamin, ${ }^{6}$ M. Benoit, ${ }^{54}$ J. R. Bensinger, ${ }^{26}$ S. Bentvelsen, ${ }^{120}$ L. Beresford, ${ }^{135}$ M. Beretta,${ }^{51}$ D. Berge, ${ }^{46}$

E. Bergeaas Kuutmann, ${ }^{172}$ N. Berger, ${ }^{5}$ B. Bergmann, ${ }^{142}$ L. J. Bergsten, ${ }^{26}$ J. Beringer, ${ }^{18}$ S. Berlendis, ${ }^{7}$ N. R. Bernard, ${ }^{103}$ G. Bernardi, ${ }^{136}$ C. Bernius, ${ }^{153}$ F. U. Bernlochner, ${ }^{24}$ T. Berry,${ }^{94}$ P. Berta, ${ }^{100}$ C. Bertella, ${ }^{15 a}$ I. A. Bertram, ${ }^{90}$

O. Bessidskaia Bylund, ${ }^{182}$ N. Besson, ${ }^{145}$ A. Bethani, ${ }^{101}$ S. Bethke, ${ }^{115}$ A. Betti ${ }^{24}$ A. J. Bevan, ${ }^{93}$ J. Beyer, ${ }^{115}$

D. S. Bhattacharya, ${ }^{177}$ R. Bi ${ }^{139}$ R. M. Bianchi, ${ }^{139}$ O. Biebel, ${ }^{114}$ D. Biedermann, ${ }^{19}$ R. Bielski, ${ }^{36}$ K. Bierwagen, ${ }^{100}$ N. V. Biesuz, ${ }^{72 a, 72 b}$ M. Biglietti, ${ }^{75 a}$ T. R. V. Billoud, ${ }^{110}$ M. Bindi, ${ }^{53}$ A. Bingul, ${ }^{12 d}$ C. Bini, ${ }^{73 a, 73 b}$ S. Biondi, ${ }^{23 b, 23 a}$ M. Birman, ${ }^{180}$ T. Bisanz, ${ }^{53}$ J. P. Biswal, ${ }^{161}$ D. Biswas, ${ }^{181, e}$ A. Bitadze, ${ }^{101}$ C. Bittrich,${ }^{48}$ K. Bjørke, ${ }^{134}$ K. M. Black, ${ }^{25}$ T. Blazek,${ }^{28 a}$ I. Bloch, ${ }^{46}$ C. Blocker, ${ }^{26}$ A. Blue, ${ }^{57}$ U. Blumenschein, ${ }^{93}$ G. J. Bobbink, ${ }^{120}$ V. S. Bobrovnikov, ${ }^{12 b, 122 a}$ S. S. Bocchetta, ${ }^{97}$ A. Bocci, ${ }^{49}$ D. Boerner, ${ }^{46}$ D. Bogavac, ${ }^{14}$ A. G. Bogdanchikov, ${ }^{122 b, 122 a}$ C. Bohm,${ }^{45 a}$ V. Boisvert, ${ }^{94}$ P. Bokan,${ }^{53,172,53}$ T. Bold, ${ }^{84 a}$ A. S. Boldyrev, ${ }^{113}$ A. E. Bolz, ${ }^{61 b}$ M. Bomben, ${ }^{136}$ M. Bona, ${ }^{93}$ J. S. Bonilla, ${ }^{132}$ M. Boonekamp, ${ }^{145}$ H. M. Borecka-Bielska, ${ }^{91}$ A. Borisov, ${ }^{123}$ G. Borissov, ${ }^{90}$ J. Bortfeldt,${ }^{36}$ D. Bortoletto, ${ }^{135}$ D. Boscherini, ${ }^{23 b}$ M. Bosman, ${ }^{14}$ J. D. Bossio Sola, ${ }^{104}$ K. Bouaouda, ${ }^{35 a}$ J. Boudreau, ${ }^{139}$ E. V. Bouhova-Thacker, ${ }^{90}$ D. Boumediene, ${ }^{38}$ S. K. Boutle, ${ }^{57}$ A. Boveia, ${ }^{127}$ J. Boyd, ${ }^{36}$ D. Boye ${ }^{33 c, i}$ I. R. Boyko, ${ }^{80}$ A. J. Bozson, ${ }^{94}$ J. Bracinik, ${ }^{21}$ N. Brahimi, ${ }^{102}$ G. Brandt, ${ }^{182}$ O. Brandt,${ }^{32}$ F. Braren, ${ }^{46}$ B. Brau, ${ }^{103}$ J. E. Brau, ${ }^{132}$ W. D. Breaden Madden, ${ }^{57}$ K. Brendlinger ${ }^{46}$ L. Brenner ${ }^{46}$ R. Brenner, ${ }^{172}$ S. Bressler, ${ }^{180}$ B. Brickwedde, ${ }^{100}$ D. L. Briglin, ${ }^{21}$ D. Britton, ${ }^{57}$ D. Britzger, ${ }^{115}$ I. Brock,${ }^{24}$ R. Brock, ${ }^{107}$ G. Brooijmans,${ }^{39}$ W. K. Brooks ${ }^{147 d}$ E. Brost, ${ }^{121}$ J. H Broughton, ${ }^{21}$ P. A. Bruckman de Renstrom, ${ }^{85}$ D. Bruncko, ${ }^{28 b}$ A. Bruni, ${ }^{23 b}$ G. Bruni, ${ }^{23 b}$ L. S. Bruni, ${ }^{120}$ S. Bruno, ${ }^{74 a, 74 b}$ B. H. Brunt, ${ }^{32}$ M. Bruschi, ${ }^{23 b}$ N. Bruscino, ${ }^{139}$ P. Bryant, ${ }^{37}$ L. Bryngemark,${ }^{97}$ T. Buanes,${ }^{17}$ Q. Buat,${ }^{36}$ P. Buchholz, ${ }^{151}$ A. G. Buckley, ${ }^{57}$ I. A. Budagov, ${ }^{80}$ M. K. Bugge, ${ }^{134}$ F. Bührer,${ }^{52}$ O. Bulekov, ${ }^{112}$ T. J. Burch ${ }^{121}$ S. Burdin,${ }^{91}$ C. D. Burgard,${ }^{120}$ A. M. Burger, ${ }^{130}$ B. Burghgrave, ${ }^{8}$ J. T. P. Burr, ${ }^{46}$ C. D. Burton, ${ }^{11}$ J. C. Burzynski, ${ }^{103}$ V. Büscher, ${ }^{100}$ E. Buschmann,${ }^{53}$ P. J. Bussey, ${ }^{57}$ J. M. Butler, ${ }^{25}$ C. M. Buttar, ${ }^{57}$ J. M. Butterworth, ${ }^{95}$ P. Butti,${ }^{36}$ W. Buttinger,${ }^{36}$ C. J. Buxo Vazquez, ${ }^{107}$ A. Buzatu, ${ }^{158}$ A. R. Buzykaev, ${ }^{122 b, 122 a}$ G. Cabras, ${ }^{23 b, 23 a}$ S. Cabrera Urbán, ${ }^{174}$ D. Caforio, ${ }^{56}$ H. Cai, ${ }^{173}$ V. M. M. Cairo, ${ }^{153}$ O. Cakir, ${ }^{4 a}$ N. Calace, ${ }^{36}$ P. Calafiura, ${ }^{18}$ A. Calandri, ${ }^{102}$ G. Calderini, ${ }^{136}$ P. Calfayan, ${ }^{66}$ G. Callea, ${ }^{57}$ L. P. Caloba, ${ }^{81 b}$ S. Calvente Lopez, ${ }^{99}$ D. Calvet, ${ }^{38}$ S. Calvet, ${ }^{38}$ T. P. Calvet, ${ }^{155}$ M. Calvetti, ${ }^{72 a, 72 b}$ R. Camacho Toro, ${ }^{136}$ S. Camarda, ${ }^{36}$ D. Camarero Munoz, ${ }^{99}$ P. Camarri, ${ }^{74 a, 74 b}$ D. Cameron, ${ }^{134}$ R. Caminal Armadans, ${ }^{103}$ C. Camincher, ${ }^{36}$ S. Campana, ${ }^{36}$ M. Campanelli, ${ }^{95}$ A. Camplani, ${ }^{40}$ A. Campoverde ${ }^{151}$ V. Canale,${ }^{70 a, 70 b}$ A. Canesse, ${ }^{104}$ M. Cano Bret, ${ }^{60 \mathrm{c}}$ J. Cantero, ${ }^{130}$ T. Cao, ${ }^{161}$ Y. Cao, ${ }^{173}$ M. D. M. Capeans Garrido, ${ }^{36}$ M. Capua, ${ }^{41 \mathrm{~b}, 41 \mathrm{a}}$ R. Cardarelli, ${ }^{74 a}$ F. Cardillo, ${ }^{149}$ G. Carducci, ${ }^{41 b, 41 \mathrm{a}}$ I. Carli, ${ }^{143}$ T. Carli, ${ }^{36}$ G. Carlino, ${ }^{70 \mathrm{a}}$ B. T. Carlson, ${ }^{139}$ L. Carminati, ${ }^{69,69 \mathrm{~b}}$ R. M. D. Carney, ${ }^{45,45 \mathrm{~b}}$ S. Caron, ${ }^{119}$ E. Carquin, ${ }^{147 \mathrm{~d}}$ S. Carrá, ${ }^{46}$ J. W. S. Carter, ${ }^{167}$ M. P. Casado, ${ }^{14, j}$ A. F. Casha,${ }^{167}$ D. W. Casper, ${ }^{171}$ R. Castelijn, ${ }^{120}$ F. L. Castillo, ${ }^{174}$ V. Castillo Gimenez, ${ }^{174}$ N. F. Castro, ${ }^{140 a, 140 \mathrm{e}}$ A. Catinaccio, ${ }^{36}$ J. R. Catmore, ${ }^{134}$ A. Cattai, ${ }^{36}$ J. Caudron,${ }^{24}$ V. Cavaliere ${ }^{29}$ E. Cavallaro, ${ }^{14}$ M. Cavalli-Sforza, ${ }^{14}$ V. Cavasinni, ${ }^{72 a, 72 b}$ E. Celebi, ${ }^{12 b}$ F. Ceradini, ${ }^{75 a, 75 b}$ L. Cerda Alberich, ${ }^{174}$ K. Cerny, ${ }^{131}$ A. S. Cerqueira, ${ }^{81 \mathrm{a}}$ A. Cerri, ${ }^{156}$ L. Cerrito, ${ }^{74 a, 74 b}$ F. Cerutti, ${ }^{18}$ A. Cervelli, ${ }^{23 b, 23 a}$ S. A. Cetin, ${ }^{12 b}$ Z. Chadi, ${ }^{35 a}$ D. Chakraborty, ${ }^{121}$ S. K. Chan, ${ }^{59}$ W. S. Chan, ${ }^{120}$ W. Y. Chan, ${ }^{91}$ J. D. Chapman, ${ }^{32}$ B. Chargeishvili, ${ }^{159 b}$ D. G. Charlton, ${ }^{21}$ T. P. Charman, ${ }^{93}$ C. C. Chau, ${ }^{34}$ S. Che, ${ }^{127}$ S. Chekanov, ${ }^{6}$ S. V. Chekulaev, ${ }^{168 a}$ G. A. Chelkov, ${ }^{80, k}$ M. A. Chelstowska, ${ }^{36}$ B. Chen,${ }^{79}$ C. Chen, ${ }^{60 a}$ C. H. Chen ${ }^{79}$ H. Chen, ${ }^{29}$ J. Chen, ${ }^{60 a}$ J. Chen, ${ }^{39}$ S. Chen, ${ }^{137}$ S. J. Chen, ${ }^{15 c}$ X. Chen, ${ }^{15 b, 1}$ Y. Chen, ${ }^{83}$ Y-H. Chen, ${ }^{46}$ H. C. Cheng, ${ }^{63 a}$ H. J. Cheng, ${ }^{15 a}$ A. Cheplakov, ${ }^{80}$ E. Cheremushkina, ${ }^{123}$ R. Cherkaoui El Moursli, ${ }^{35 e}$ E. Cheu, ${ }^{7}$ K. Cheung, ${ }^{64}$ 
T. J. A. Chevalérias, ${ }^{145}$ L. Chevalier, ${ }^{145}$ V. Chiarella, ${ }^{51}$ G. Chiarelli, ${ }^{72 a}$ G. Chiodini, ${ }^{68 a}$ A. S. Chisholm, ${ }^{21}$ A. Chitan, ${ }^{27 b}$ I. Chiu, ${ }^{163}$ Y. H. Chiu, ${ }^{176}$ M. V. Chizhov, ${ }^{80}$ K. Choi, ${ }^{66}$ A. R. Chomont, ${ }^{73 a, 73 b}$ S. Chouridou, ${ }^{162}$ Y. S. Chow, ${ }^{120}$ M. C. Chu, ${ }^{63 a}$ X. Chu, ${ }^{15 a, 15 d}$ J. Chudoba, ${ }^{141}$ A. J. Chuinard, ${ }^{104}$ J. J. Chwastowski, ${ }^{85}$ L. Chytka,${ }^{131}$ D. Cieri, ${ }^{115}$ K. M. Ciesla, ${ }^{85}$ D. Cinca, ${ }^{47}$

V. Cindro, ${ }^{92}$ I. A. Cioară, ${ }^{27 b}$ A. Ciocio, ${ }^{18}$ F. Cirotto, ${ }^{70 a, 70 b}$ Z. H. Citron, ${ }^{180, m}$ M. Citterio, ${ }^{69 \mathrm{a}}$ D. A. Ciubotaru, ${ }^{27 b}$ B. M. Ciungu, ${ }^{167}$ A. Clark, ${ }^{54}$ M. R. Clark, ${ }^{39}$ P. J. Clark, ${ }^{50}$ C. Clement, ${ }^{45 a, 45 b}$ Y. Coadou, ${ }^{102}$ M. Cobal,,${ }^{67,67 c}$ A. Coccaro, ${ }^{55 b}$ J. Cochran, ${ }^{79}$ H. Cohen, ${ }^{161}$ A. E. C. Coimbra, ${ }^{36}$ L. Colasurdo, ${ }^{119}$ B. Cole, ${ }^{39}$ A. P. Colijn, ${ }^{120}$ J. Collot, ${ }^{58}$ P. Conde Muiño, ${ }^{140 a, n}$ E. Coniavitis, ${ }^{52}$ S. H. Connell, ${ }^{33 \mathrm{c}}$ I. A. Connelly, ${ }^{57}$ S. Constantinescu, ${ }^{27 b}$ F. Conventi, ${ }^{70 a, 0}$ A. M. Cooper-Sarkar, ${ }^{135}$ F. Cormier, ${ }^{175}$ K. J. R. Cormier, ${ }^{167}$ L. D. Corpe, ${ }^{95}$ M. Corradi, ${ }^{73 a, 73 b}$ E. E. Corrigan,${ }^{97}$ F. Corriveau, ${ }^{104, p}$ M. J. Costa, ${ }^{174}$ F. Costanza, ${ }^{5}$ D. Costanzo, ${ }^{149}$ G. Cowan,,${ }^{94}$ J. W. Cowley, ${ }^{32}$ J. Crane, ${ }^{101}$ K. Cranmer, ${ }^{125}$ S. J. Crawley, ${ }^{57}$ R. A. Creager, ${ }^{137}$ S. Crépé-Renaudin, ${ }^{58}$ F. Crescioli, ${ }^{136}$ M. Cristinziani, ${ }^{24}$ V. Croft, ${ }^{120}$ G. Crosetti, ${ }^{41 b, 41 a}$ A. Cueto,

T. Cuhadar Donszelmann, ${ }^{149}$ A. R. Cukierman, ${ }^{153}$ S. Czekierda, ${ }^{85}$ P. Czodrowski, ${ }^{36}$ M. J. Da Cunha Sargedas De Sousa, ${ }^{60 b}$ J. V. Da Fonseca Pinto, ${ }^{81 b}$ C. Da Via, ${ }^{101}$ W. Dabrowski, ${ }^{84 a}$ T. Dado ${ }^{28 a}$ S. Dahbi, ${ }^{35 e}$ T. Dai, ${ }^{106}$ C. Dallapiccola,${ }^{103}$ M. Dam, ${ }^{40}$

G. D'amen, ${ }^{29}$ V. D’Amico, ${ }^{75 a, 75 b}$ J. Damp, ${ }^{100}$ J. R. Dandoy ${ }^{137}$ M. F. Daneri, ${ }^{30}$ N. P. Dang, ${ }^{181, e}$ N. S. Dann, ${ }^{101}$

M. Danninger, ${ }^{175}$ V. Dao, ${ }^{36}$ G. Darbo, ${ }^{55 b}$ O. Dartsi,${ }^{5}$ A. Dattagupta, ${ }^{132}$ T. Daubney, ${ }^{46}$ S. D’ Auria,${ }^{69 a, 69 b}$ W. Davey,${ }^{24}$ C. David, ${ }^{46}$ T. Davidek, ${ }^{143}$ D. R. Davis, ${ }^{49}$ I. Dawson, ${ }^{149}$ K. De,${ }^{8}$ R. De Asmundis, ${ }^{70 a}$ M. De Beurs, ${ }^{120}$ S. De Castro, ${ }^{23 b, 23 a}$ S. De Cecco, ${ }^{73 a, 73 b}$ N. De Groot, ${ }^{119}$ P. de Jong, ${ }^{120}$ H. De la Torre, ${ }^{107}$ A. De Maria, ${ }^{15 c}$ D. De Pedis, ${ }^{73 a}$ A. De Salvo, ${ }^{73 a}$ U. De Sanctis ${ }^{74 a, 74 b}$ M. De Santis, ${ }^{74 a, 74 b}$ A. De Santo, ${ }^{156}$ K. De Vasconcelos Corga, ${ }^{102}$ J. B. De Vivie De Regie, ${ }^{65}$ C. Debenedetti, ${ }^{146}$ D. V. Dedovich, ${ }^{80}$ A. M. Deiana,${ }^{42}$ M. Del Gaudio, ${ }^{41 b, 41 a}$ J. Del Peso, ${ }^{99}$ Y. Delabat Diaz,${ }^{46}$ D. Delgove,${ }^{65}$ F. Deliot, ${ }^{145, q}$ C. M. Delitzsch, ${ }^{7}$ M. Della Pietra, ${ }^{70 a, 70 b}$ D. Della Volpe,${ }^{54}$ A. Dell'Acqua,${ }^{36}$ L. Dell'Asta, ${ }^{74 a, 74 b}$ M. Delmastro, ${ }^{5}$ C. Delporte, ${ }^{65}$ P. A. Delsart, ${ }^{58}$ D. A. DeMarco, ${ }^{167}$ S. Demers, ${ }^{183}$ M. Demichev, ${ }^{80}$ G. Demontigny, ${ }^{110}$ S. P. Denisov, ${ }^{123}$ D. Denysiuk, ${ }^{120}$ L. D’Eramo, ${ }^{136}$ D. Derendarz, ${ }^{85}$ J. E. Derkaoui, ${ }^{35 d}$ F. Derue,${ }^{136}$ P. Dervan, ${ }^{91}$ K. Desch, ${ }^{24}$ C. Deterre, ${ }^{46}$ K. Dette, ${ }^{167}$ C. Deutsch, ${ }^{24}$ M. R. Devesa,${ }^{30}$ P. O. Deviveiros, ${ }^{36}$ A. Dewhurst,${ }^{144}$ F. A. Di Bello, ${ }^{54}$ A. Di Ciaccio, ${ }^{74 a, 74 b}$ L. Di Ciaccio, ${ }^{5}$ W. K. Di Clemente, ${ }^{137}$ C. Di Donato, ${ }^{70 a, 70 b}$ A. Di Girolamo, ${ }^{36}$ G. Di Gregorio, ${ }^{72 a, 72 b}$ B. Di Micco, ${ }^{75 a, 75 b}$ R. Di Nardo, ${ }^{103}$ K. F. Di Petrillo, ${ }^{59}$ R. Di Sipio, ${ }^{167}$ D. Di Valentino, ${ }^{34}$ C. Diaconu,${ }^{102}$ F. A. Dias, ${ }^{40}$ T. Dias Do Vale, ${ }^{140 a}$ M. A. Diaz, ${ }^{147 a}$ J. Dickinson, ${ }^{18}$ E. B. Diehl,${ }^{106}$ J. Dietrich, ${ }^{19}$ S. Díez Cornell, ${ }^{46}$ A. Dimitrievska, ${ }^{18}$ W. Ding, ${ }^{15 b}$ J. Dingfelder, ${ }^{24}$ F. Dittus,${ }^{36}$ F. Djama, ${ }^{102}$ T. Djobava, ${ }^{159 b}$ J. I. Djuvsland,${ }^{17}$ M. A. B. Do Vale, ${ }^{81 \mathrm{c}}$ M. Dobre, ${ }^{27 b}$ D. Dodsworth, ${ }^{26}$ C. Doglioni, ${ }^{97}$ J. Dolejsi, ${ }^{143}$ Z. Dolezal,${ }^{143}$ M. Donadelli ${ }^{81 d}$ B. Dong, ${ }^{60 \mathrm{c}}$ J. Donini, ${ }^{38}$ A. D'onofrio, ${ }^{93}$ M. D’Onofrio, ${ }^{91}$ J. Dopke, ${ }^{144}$ A. Doria, ${ }^{70 a}$ M. T. Dova, ${ }^{89}$ A. T. Doyle, ${ }^{57}$ E. Drechsler, ${ }^{152}$ E. Dreyer, ${ }^{152}$ T. Dreyer, ${ }^{53}$ A. S. Drobac, ${ }^{170}$ D. Du, ${ }^{60 \mathrm{~b}}$ Y. Duan, ${ }^{60 \mathrm{~b}}$ F. Dubinin, ${ }^{111}$ M. Dubovsky, ${ }^{28 \mathrm{a}}$ A. Dubreuil,${ }^{54}$ E. Duchovni, ${ }^{180}$ G. Duckeck, ${ }^{114}$ A. Ducourthial, ${ }^{136}$ O. A. Ducu, ${ }^{110}$ D. Duda,${ }^{115}$ A. Dudarev,${ }^{36}$ A. C. Dudder, ${ }^{100}$ E. M. Duffield, ${ }^{18}$ L. Duflot,${ }^{65}$ M. Dührssen,${ }^{36}$ C. Dülsen, ${ }^{182}$ M. Dumancic, ${ }^{180}$ A. E. Dumitriu, ${ }^{27 b}$ A. K. Duncan, ${ }^{57}$ M. Dunford, ${ }^{61 a}$ A. Duperrin, ${ }^{102}$ H. Duran Yildiz, ${ }^{4 a}$ M. Düren, ${ }^{56}$ A. Durglishvili, ${ }^{159 b}$ D. Duschinger, ${ }^{48}$ B. Dutta, ${ }^{46}$ D. Duvnjak, ${ }^{1}$ G. I. Dyckes,${ }^{137}$ M. Dyndal, ${ }^{36}$ S. Dysch,${ }^{101}$ B. S. Dziedzic ${ }^{85}$ K. M. Ecker, ${ }^{115}$ R. C. Edgar, ${ }^{106}$ M. G. Eggleston, ${ }^{49}$ T. Eifert, ${ }^{36}$ G. Eigen, ${ }^{17}$ K. Einsweiler,${ }^{18}$ T. Ekelof, ${ }^{172}$ H. El Jarrari, ${ }^{35 \mathrm{e}} \mathrm{M}$. El Kacimi, ${ }^{35 \mathrm{c}}$ R. El Kosseifi, ${ }^{102}$ V. Ellajosyula, ${ }^{172}$ M. Ellert, ${ }^{172}$ F. Ellinghaus, ${ }^{182}$ A. A. Elliot, ${ }^{93}$ N. Ellis,${ }^{36}$ J. Elmsheuser, ${ }^{29}$ M. Elsing, ${ }^{36}$ D. Emeliyanov, ${ }^{144}$ A. Emerman, ${ }^{39}$ Y. Enari, ${ }^{163}$ M. B. Epland, ${ }^{49}$ J. Erdmann, ${ }^{47}$ A. Ereditato, ${ }^{20}$ M. Errenst, ${ }^{36}$ M. Escalier, ${ }^{65}$ C. Escobar, ${ }^{174}$ O. Estrada Pastor, ${ }^{174}$ E. Etzion, ${ }^{161}$ H. Evans, ${ }^{66}$ A. Ezhilov, ${ }^{138}$ F. Fabbri, ${ }^{57}$ L. Fabbri, ${ }^{23 b, 23 a}$ V. Fabiani, ${ }^{119}$ G. Facini, ${ }^{95}$ R. M. Faisca Rodrigues Pereira, ${ }^{140 a}$ R. M. Fakhrutdinov, ${ }^{123}$ S. Falciano, ${ }^{73 a}$ P. J. Falke, ${ }^{5}$ S. Falke, ${ }^{5}$ J. Faltova, ${ }^{143}$ Y. Fang, ${ }^{15 a}$ Y. Fang, ${ }^{15 a}$ G. Fanourakis,${ }^{44}$ M. Fanti, ${ }^{69 a, 69 b}$ M. Faraj, ${ }^{67 a, 67 c, r}$ A. Farbin, ${ }^{8}$ A. Farilla, ${ }^{75 a}$ E. M. Farina, ${ }^{71 a, 71 b}$ T. Farooque, ${ }^{107}$ S. Farrell, ${ }^{18}$ S. M. Farrington, ${ }^{50}$ P. Farthouat, ${ }^{36}$ F. Fassi,${ }^{35 e}$ P. Fassnacht,${ }^{36}$ D. Fassouliotis, ${ }^{9}$ M. Faucci Giannelli, ${ }^{50}$ W. J. Fawcett, ${ }^{32}$ L. Fayard, ${ }^{65}$ O. L. Fedin, ${ }^{138, s}$ W. Fedorko, ${ }^{175}$ M. Feickert, ${ }^{42}$ L. Feligioni ${ }^{102}$ A. Fell, ${ }^{149}$ C. Feng, ${ }^{60 b}$ E. J. Feng, ${ }^{36}$ M. Feng, ${ }^{49}$ M. J. Fenton, ${ }^{57}$ A. B. Fenyuk, ${ }^{123}$ J. Ferrando,${ }^{46}$ A. Ferrante, ${ }^{173}$ A. Ferrari, ${ }^{172}$ P. Ferrari, ${ }^{120}$ R. Ferrari, ${ }^{71 a}$ D. E. Ferreira de Lima, ${ }^{61 b}$ A. Ferrer, ${ }^{174}$ D. Ferrere, ${ }^{54}$ C. Ferretti, ${ }^{106}$ F. Fiedler, ${ }^{100}$ A. Filipčič, ${ }^{92}$ F. Filthaut, ${ }^{119}$ K. D. Finelli, ${ }^{25}$ M. C. N. Fiolhais, ${ }^{140 a, 140 c, t}$ L. Fiorini, ${ }^{174}$ F. Fischer, ${ }^{114}$ W. C. Fisher,${ }^{107}$ I. Fleck, ${ }^{151}$ P. Fleischmann, ${ }^{106}$ R. R. M. Fletcher, ${ }^{137}$ T. Flick, ${ }^{182}$ B. M. Flierl, ${ }^{114}$ L. Flores,${ }^{137}$ L. R. Flores Castillo, ${ }^{63 \mathrm{a}}$ F. M. Follega, ${ }^{76 a, 76 b}$ N. Fomin, ${ }^{17}$ J. H. Foo, ${ }^{167}$ G. T. Forcolin, ${ }^{76 a, 76 b}$ A. Formica, ${ }^{145}$ F. A. Förster, ${ }^{14}$ A. C. Forti, ${ }^{101}$ A. G. Foster, ${ }^{21}$ M. G. Foti, ${ }^{135}$ D. Fournier, ${ }^{65}$ H. Fox,${ }^{90}$ P. Francavilla, ${ }^{72 a, 72 b}$ S. Francescato, ${ }^{73 a, 73 b}$ M. Franchini,${ }^{23 b, 23 a}$ S. Franchino, ${ }^{61 a}$ D. Francis, ${ }^{36}$

L. Franconi, ${ }^{20}$ M. Franklin, ${ }^{59}$ A. N. Fray,${ }^{93}$ P. M. Freeman, ${ }^{21}$ B. Freund, ${ }^{110}$ W. S. Freund, ${ }^{81 b}$ E. M. Freundlich, ${ }^{47}$

D. C. Frizzell, ${ }^{129}$ D. Froidevaux, ${ }^{36}$ J. A. Frost, ${ }^{135}$ C. Fukunaga, ${ }^{164}$ E. Fullana Torregrosa, ${ }^{174}$ E. Fumagalli, ${ }^{55 b, 55 a}$

T. Fusayasu, ${ }^{16}$ J. Fuster, ${ }^{174}$ A. Gabrielli, ${ }^{23 b, 23 a}$ A. Gabrielli, ${ }^{18}$ G. P. Gach,${ }^{84 a}$ S. Gadatsch,${ }^{54}$ P. Gadow, ${ }^{115}$ G. Gagliardi, ${ }^{55,55 a}$ 
L. G. Gagnon, ${ }^{110}$ C. Galea, ${ }^{27 b}$ B. Galhardo, ${ }^{140 a}$ G. E. Gallardo, ${ }^{135}$ E. J. Gallas, ${ }^{135}$ B. J. Gallop, ${ }^{144}$ G. Galster, ${ }^{40}$ R. Gamboa Goni, ${ }^{93}$ K. K. Gan, ${ }^{127}$ S. Ganguly, ${ }^{180}$ J. Gao, ${ }^{60 a}$ Y. Gao, ${ }^{50}$ Y. S. Gao, ${ }^{31, g}$ C. García, ${ }^{174}$ J. E. García Navarro, ${ }^{174}$ J. A. García Pascual, ${ }^{15 a}$ C. Garcia-Argos,${ }^{52}$ M. Garcia-Sciveres, ${ }^{18}$ R. W. Gardner, ${ }^{37}$ N. Garelli, ${ }^{153}$ S. Gargiulo, ${ }^{52}$

V. Garonne, ${ }^{134}$ A. Gaudiello, ${ }^{55,55 a}$ G. Gaudio, ${ }^{71 a}$ I. L. Gavrilenko, ${ }^{111}$ A. Gavrilyuk, ${ }^{124}$ C. Gay, ${ }^{175}$ G. Gaycken, ${ }^{46}$ E. N. Gazis, ${ }^{10}$ A. A. Geanta, ${ }^{27 b}$ C. M. Gee, ${ }^{146}$ C. N. P. Gee, ${ }^{144}$ J. Geisen, ${ }^{53}$ M. Geisen, ${ }^{100}$ M. P. Geisler, ${ }^{61 a}$ C. Gemme,${ }^{55 b}$ M. H. Genest, ${ }^{58}$ C. Geng, ${ }^{106}$ S. Gentile, ${ }^{73 a, 73 b}$ S. George, ${ }^{94}$ T. Geralis, ${ }^{44}$ L. O. Gerlach, ${ }^{53}$ P. Gessinger-Befurt, ${ }^{100}$ G. Gessner, ${ }^{47}$ S. Ghasemi, ${ }^{151}$ M. Ghasemi Bostanabad ${ }^{176}$ A. Ghosh, ${ }^{65}$ A. Ghosh,${ }^{78}$ B. Giacobbe, ${ }^{23 b}$ S. Giagu, ${ }^{73 a, 73 b}$ N. Giangiacomi, ${ }^{23 b, 23 a}$ P. Giannetti, ${ }^{72 a}$ A. Giannini, ${ }^{7 a, 70 b}$ G. Giannini, ${ }^{14}$ S. M. Gibson, ${ }^{94}$ M. Gignac, ${ }^{146}$ D. Gillberg, ${ }^{34}$ G. Gilles, ${ }^{182}$ D. M. Gingrich, ${ }^{3, d}$ M. P. Giordani, ${ }^{67 a, 67 c}$ F. M. Giorgi, ${ }^{23 b}$ P. F. Giraud,${ }^{145}$ G. Giugliarelli, ${ }^{67 a, 67 c}$ D. Giugni,${ }^{69 a}$ F. Giuli, ${ }^{74 a, 74 b}$ S. Gkaitatzis, ${ }^{162}$ I. Gkialas, ${ }^{9, u}$ E. L. Gkougkousis,${ }^{14}$ P. Gkountoumis, ${ }^{10}$ L. K. Gladilin, ${ }^{113}$ C. Glasman, ${ }^{99}$ J. Glatzer, ${ }^{14}$ P. C. F. Glaysher, ${ }^{46}$ A. Glazov, ${ }^{46}$ G. R. Gledhill, ${ }^{132}$ M. Goblirsch-Kolb, ${ }^{26}$ D. Godin, ${ }^{110}$ S. Goldfarb,${ }^{105}$ T. Golling, ${ }^{54}$ D. Golubkov, ${ }^{123}$ A. Gomes, ${ }^{140 a, 140 b}$ R. Goncalves Gama, ${ }^{53}$ R. Gonçalo, ${ }^{140 a}$ G. Gonella, ${ }^{52}$ L. Gonella, ${ }^{21}$ A. Gongadze ${ }^{80}$ F. Gonnella, ${ }^{21}$ J. L. Gonski, ${ }^{59}$ S. González de la Hoz, ${ }^{174}$ S. Gonzalez-Sevilla, ${ }^{54}$

G. R. Gonzalvo Rodriguez, ${ }^{174}$ L. Goossens, ${ }^{36}$ P. A. Gorbounov, ${ }^{124}$ H. A. Gordon, ${ }^{29}$ B. Gorini, ${ }^{36}$ E. Gorini, ${ }^{68 a, 68 b}$ A. Gorišek, ${ }^{92}$ A. T. Goshaw, ${ }^{49}$ M. I. Gostkin, ${ }^{80}$ C. A. Gottardo, ${ }^{119}$ M. Gouighri, ${ }^{35 b}$ D. Goujdami, ${ }^{35 c}$ A. G. Goussiou, ${ }^{148}$ N. Govender, ${ }^{33 \mathrm{c}}$ C. Goy, ${ }^{5}$ E. Gozani, ${ }^{160}$ I. Grabowska-Bold, ${ }^{84 a}$ E. C. Graham, ${ }^{91}$ J. Gramling, ${ }^{171}$ E. Gramstad, ${ }^{134}$ S. Grancagnolo, ${ }^{19}$ M. Grandi, ${ }^{156}$ V. Gratchev ${ }^{138}$ P. M. Gravila, ${ }^{27 f}$ F. G. Gravili, ${ }^{68 a, 68 b}$ C. Gray,${ }^{57}$ H. M. Gray, ${ }^{18}$ C. Grefe, ${ }^{24}$ K. Gregersen, ${ }^{97}$ I. M. Gregor, ${ }^{46}$ P. Grenier, ${ }^{153}$ K. Grevtsov, ${ }^{46}$ C. Grieco, ${ }^{14}$ N. A. Grieser, ${ }^{129}$ J. Griffiths, ${ }^{8}$ A. A. Grillo, ${ }^{146}$ K. Grimm, ${ }^{31, v}$ S. Grinstein, ${ }^{14, w}$ J.-F. Grivaz, ${ }^{65}$ S. Groh, ${ }^{100}$ E. Gross, ${ }^{180}$ J. Grosse-Knetter, ${ }^{53}$ Z. J. Grout, ${ }^{95}$ C. Grud, ${ }^{106}$ A. Grummer, ${ }^{118}$ L. Guan, ${ }^{106}$ W. Guan, ${ }^{181}$ J. Guenther, ${ }^{36}$ A. Guerguichon, ${ }^{65}$ J. G. R. Guerrero Rojas, ${ }^{174}$ F. Guescini, ${ }^{115}$ D. Guest, ${ }^{171}$ R. Gugel, ${ }^{52}$ T. Guillemin, ${ }^{5}$ S. Guindon, ${ }^{36}$ U. Gul, ${ }^{57}$ J. Guo, ${ }^{60 \mathrm{c}}$ W. Guo, ${ }^{106}$ Y. Guo, ${ }^{60 a, \mathrm{x}}$ Z. Guo, ${ }^{102}$ R. Gupta, ${ }^{46}$ S. Gurbuz, ${ }^{12 c}$ G. Gustavino, ${ }^{129}$ M. Guth, ${ }^{52}$ P. Gutierrez, ${ }^{129}$ C. Gutschow,${ }^{95}$ C. Guyot, ${ }^{145}$ C. Gwenlan, ${ }^{135}$ C. B. Gwilliam, ${ }^{91}$ A. Haas, ${ }^{125}$ C. Haber, ${ }^{18}$ H. K. Hadavand ${ }^{8}$ N. Haddad, ${ }^{35 e}$ A. Hadef,${ }^{60 a}$ S. Hageböck, ${ }^{36}$ M. Haleem, ${ }^{177}$ J. Haley, ${ }^{130}$ G. Halladjian, ${ }^{107}$ G. D. Hallewell, ${ }^{102}$ K. Hamacher, ${ }^{182}$ P. Hamal, ${ }^{131}$ K. Hamano,${ }^{176}$ H. Hamdaoui, ${ }^{35 e}$ G. N. Hamity, ${ }^{149}$ K. Han, ${ }^{60 a, y}$ L. Han, ${ }^{60 a}$ S. Han, ${ }^{15 a}$ Y. F. Han, ${ }^{167}$ K. Hanagaki ${ }^{82, z}$ M. Hance,${ }^{146}$ D. M. Handl, ${ }^{114}$ B. Haney, ${ }^{137}$ R. Hankache, ${ }^{136}$ E. Hansen, ${ }^{97}$ J. B. Hansen, ${ }^{40}$ J. D. Hansen, ${ }^{40}$ M. C. Hansen, ${ }^{24}$ P. H. Hansen, ${ }^{40}$ E. C. Hanson, ${ }^{101}$ K. Hara, ${ }^{169}$ T. Harenberg, ${ }^{182}$ S. Harkusha, ${ }^{108}$ P. F. Harrison, ${ }^{178}$ N. M. Hartmann, ${ }^{114}$ Y. Hasegawa, ${ }^{150}$ A. Hasib,${ }^{50}$ S. Hassani, ${ }^{145}$ S. Haug, ${ }^{20}$ R. Hauser, ${ }^{107}$ L. B. Havener, ${ }^{39}$ M. Havranek, ${ }^{142}$ C. M. Hawkes ${ }^{21}$ R. J. Hawkings, ${ }^{36}$ D. Hayden, ${ }^{107}$ C. Hayes,${ }^{155}$ R. L. Hayes, ${ }^{175}$ C. P. Hays, ${ }^{135}$ J. M. Hays, ${ }^{93}$ H. S. Hayward, ${ }^{91}$ S. J. Haywood, ${ }^{144}$ F. He,${ }^{60}$ M. P. Heath,${ }^{50}$ V. Hedberg, ${ }^{97}$ L. Heelan, ${ }^{8}$ S. Heer,${ }^{24}$ K. K. Heidegger, ${ }^{52}$ W. D. Heidorn, ${ }^{79}$ J. Heilman, ${ }^{34}$ S. Heim, ${ }^{46}$ T. Heim, ${ }^{18}$ B. Heinemann, ${ }^{46, a a}$ J. J. Heinrich, ${ }^{132}$ L. Heinrich,${ }^{36}$ C. Heinz,${ }^{56}$ J. Hejbal, ${ }^{141}$ L. Helary, ${ }^{61 b}$ A. Held, ${ }^{175}$ S. Hellesund,${ }^{134}$ C. M. Helling, ${ }^{146}$ S. Hellman, ${ }^{45 a, 45 b}$ C. Helsens, ${ }^{36}$ R. C. W. Henderson, ${ }^{90}$ Y. Heng, ${ }^{181}$ S. Henkelmann, ${ }^{175}$ A. M. Henriques Correia,${ }^{36}$ G. H. Herbert,,${ }^{19}$ H. Herde,${ }^{26}$ V. Herget, ${ }^{177}$ Y. Hernández Jiménez, ${ }^{33}$ H. Herr, ${ }^{100}$ M. G. Herrmann, ${ }^{114}$ T. Herrmann, ${ }^{48}$ G. Herten, ${ }^{52}$ R. Hertenberger, ${ }^{114}$ L. Hervas, ${ }^{36}$

T. C. Herwig, ${ }^{137}$ G. G. Hesketh, ${ }^{95}$ N. P. Hessey, ${ }^{168 a}$ A. Higashida, ${ }^{163}$ S. Higashino ${ }^{82}$ E. Higón-Rodriguez, ${ }^{174}$

K. Hildebrand, ${ }^{37}$ E. Hill,${ }^{176}$ J. C. Hill,,${ }^{32}$ K. K. Hill, ${ }^{29}$ K. H. Hiller, ${ }^{46}$ S. J. Hillier ${ }^{21}$ M. Hils,${ }^{48}$ I. Hinchliffe, ${ }^{18}$ F. Hinterkeuser, ${ }^{24}$ M. Hirose, ${ }^{133}$ S. Hirose, ${ }^{52}$ D. Hirschbuehl, ${ }^{182}$ B. Hiti, ${ }^{92}$ O. Hladik, ${ }^{141}$ D. R. Hlaluku, ${ }^{33 e}$ X. Hoad, ${ }^{50}$ J. Hobbs, ${ }^{155}$ N. Hod, ${ }^{180}$ M. C. Hodgkinson, ${ }^{149}$ A. Hoecker, ${ }^{36}$ F. Hoenig, ${ }^{114}$ D. Hohn, ${ }^{52}$ D. Hohov, ${ }^{65}$ T. R. Holmes, ${ }^{37}$ M. Holzbock, ${ }^{114}$ L. B. A. H. Hommels, ${ }^{32}$ S. Honda, ${ }^{169}$ T. M. Hong, ${ }^{139}$ J. C. Honig, ${ }^{52}$ A. Hönle, ${ }^{115}$ B. H. Hooberman, ${ }^{173}$ W. H. Hopkins, ${ }^{6}$ Y. Horii ${ }^{117}$ P. Horn, ${ }^{48}$ L. A. Horyn, ${ }^{37}$ S. Hou, ${ }^{158}$ A. Hoummada, ${ }^{35 a}$ J. Howarth, ${ }^{101}$ J. Hoya,${ }^{89}$ M. Hrabovsky, ${ }^{131}$ J. Hrdinka ${ }^{77}$ I. Hristova, ${ }^{19}$ J. Hrivnac, ${ }^{65}$ A. Hrynevich, ${ }^{109}$ T. Hryn' ova, ${ }^{5}$ P. J. Hsu, ${ }^{64}$ S.-C. Hsu, ${ }^{148}$ Q. Hu, ${ }^{29}$ S. Hu ${ }^{60 \mathrm{c}}$ Y. F. Hu, ${ }^{15 a, 15 \mathrm{~d}}$ D. P. Huang, ${ }^{95}$ Y. Huang, ${ }^{60 \mathrm{a}}$ Y. Huang, ${ }^{15 \mathrm{a}}$ Z. Hubacek, ${ }^{142}$ F. Hubaut, ${ }^{102}$ M. Huebner, ${ }^{24}$ F. Huegging, ${ }^{24}$ T. B. Huffman, ${ }^{135}$ M. Huhtinen, ${ }^{36}$ R. F. H. Hunter, ${ }^{34}$ P. Huo,${ }^{155}$ A. M. Hupe,${ }^{34}$ N. Huseynov,,${ }^{80, b b}$ J. Huston,,${ }^{107}$ J. Huth, ${ }^{59}$ R. Hyneman, ${ }^{106}$ S. Hyrych, ${ }^{28 a}$ G. Iacobucci, ${ }^{54}$ G. Iakovidis, ${ }^{29}$ I. Ibragimov, ${ }^{151}$ L. Iconomidou-Fayard, ${ }^{65}$ Z. Idrissi, ${ }^{35 e}$ P. Iengo, ${ }^{36}$ R. Ignazzi, ${ }^{40}$ O. Igonkina, ${ }^{120, a, c c}$ R. Iguchi, ${ }^{163}$ T. Iizawa, ${ }^{54}$ Y. Ikegami, ${ }^{82}$ M. Ikeno, ${ }^{82}$ D. Iliadis, ${ }^{162}$ N. Ilic, ${ }^{119,167, p}$ F. Iltzsche, ${ }^{48}$ G. Introzzi, ${ }^{71 a, 71 b}$ M. Iodice, ${ }^{75 a}$ K. Iordanidou, ${ }^{168 \mathrm{a}}$ V. Ippolito, ${ }^{73 a, 73 b}$ M. F. Isacson, ${ }^{172}$ M. Ishino, ${ }^{163} \mathrm{~W}$. Islam, ${ }^{130} \mathrm{C}$. Issever, ${ }^{135} \mathrm{~S}$. Istin, ${ }^{160} \mathrm{~F}$. Ito, ${ }^{169} \mathrm{~J}$. M. Iturbe Ponce, ${ }^{63 \mathrm{a}} \mathrm{R}$. Iuppa,${ }^{76 a, 76 \mathrm{~b}} \mathrm{~A}$. Ivina, ${ }^{180} \mathrm{H}$. Iwasaki ${ }^{82}$ J. M. Izen, ${ }^{43}$ V. Izzo, ${ }^{70 a}$ P. Jacka, ${ }^{141}$ P. Jackson, ${ }^{1}$ R. M. Jacobs, ${ }^{24}$ B. P. Jaeger, ${ }^{152}$ V. Jain, ${ }^{2}$ G. Jäkel, ${ }^{182}$ K. B. Jakobi, ${ }^{100}$ K. Jakobs, ${ }^{52}$ S. Jakobsen, ${ }^{77}$ T. Jakoubek, ${ }^{141}$ J. Jamieson, ${ }^{57}$ K. W. Janas ${ }^{84 a}$ R. Jansky ${ }^{54}$ J. Janssen, ${ }^{24}$ M. Janus, ${ }^{53}$ P. A. Janus, ${ }^{84 a}$ G. Jarlskog, ${ }^{97}$ N. Javadov,${ }^{80, b b}$ T. Javůrek, ${ }^{36}$ M. Javurkova, ${ }^{52}$ F. Jeanneau, ${ }^{145}$ L. Jeanty, ${ }^{132}$ J. Jejelava, ${ }^{159 a, d d}$ 
A. Jelinskas, ${ }^{178}$ P. Jenni, ${ }^{52, e e}$ J. Jeong, ${ }^{46}$ N. Jeong, ${ }^{46}$ S. Jézéquel,,${ }^{5}$ H. Ji, ${ }^{181}$ J. Jia, ${ }^{155}$ H. Jiang, ${ }^{79}$ Y. Jiang, ${ }^{60 a}$ Z. Jiang, ${ }^{153, \text { ff }}$ S. Jiggins,${ }^{52}$ F. A. Jimenez Morales, ${ }^{38}$ J. Jimenez Pena, ${ }^{115}$ S. Jin, ${ }^{15 \mathrm{c}}$ A. Jinaru, ${ }^{27 \mathrm{~b}}$ O. Jinnouchi, ${ }^{165} \mathrm{H}$. Jivan, ${ }^{33 \mathrm{e}}$ P. Johansson, ${ }^{149}$ K. A. Johns, ${ }^{7}$ C. A. Johnson, ${ }^{66}$ K. Jon-And, ${ }^{45 a, 45 b}$ R. W. L. Jones, ${ }^{90}$ S. D. Jones, ${ }^{156}$ S. Jones, ${ }^{7}$ T. J. Jones,${ }^{91}$ J. Jongmanns, ${ }^{61 \mathrm{a}}$ P. M. Jorge, ${ }^{140 \mathrm{a}}$ J. Jovicevic, ${ }^{36} \mathrm{X}$. Ju, ${ }^{18} \mathrm{~J}$. J. Junggeburth, ${ }^{115}$ A. Juste Rozas, ${ }^{14, w}$ A. Kaczmarska, ${ }^{85}$ M. Kado, ${ }^{73 a, 73 b}$ H. Kagan, ${ }^{127}$ M. Kagan, ${ }^{153}$ C. Kahra, ${ }^{100}$ T. Kaji, ${ }^{179}$ E. Kajomovitz, ${ }^{160}$ C. W. Kalderon, ${ }^{97}$ A. Kaluza, ${ }^{100}$ A. Kamenshchikov, ${ }^{123}$ M. Kaneda, ${ }^{163}$ L. Kanjir, ${ }^{92}$ Y. Kano, ${ }^{163}$ V. A. Kantserov, ${ }^{12}$ J. Kanzaki, ${ }^{82}$ L. S. Kaplan, ${ }^{181}$ D. Kar, ${ }^{33 e}$ K. Karava, ${ }^{135}$ M. J. Kareem, ${ }^{168 b}$ S. N. Karpov, ${ }^{80}$ Z. M. Karpova, ${ }^{80}$ V. Kartvelishvili, ${ }^{90}$ A. N. Karyukhin, ${ }^{123}$ L. Kashif, ${ }^{181}$ R. D. Kass, ${ }^{127}$ A. Kastanas, ${ }^{45 a, 45 b}$ C. Kato, ${ }^{60 d, 60 c}$ J. Katzy, ${ }^{46}$ K. Kawade, ${ }^{150}$ K. Kawagoe ${ }^{88}$ T. Kawaguchi, ${ }^{117}$ T. Kawamoto, ${ }^{163}$ G. Kawamura, ${ }^{53}$ E. F. Kay, ${ }^{176}$ V. F. Kazanin, ${ }^{122 b, 122 a}$ R. Keeler, ${ }^{176}$ R. Kehoe, ${ }^{42}$ J. S. Keller, ${ }^{34}$ E. Kellermann, ${ }^{97}$ D. Kelsey, ${ }^{156}$ J. J. Kempster, ${ }^{21}$ J. Kendrick, ${ }^{21}$ O. Kepka, ${ }^{141}$ S. Kersten, ${ }^{182}$ B. P. Kerševan, ${ }^{92}$ S. Ketabchi Haghighat, ${ }^{167}$ M. Khader, ${ }^{173}$ F. Khalil-Zada, ${ }^{13}$ M. Khandoga, ${ }^{145}$ A. Khanov, ${ }^{130}$ A. G. Kharlamov, ${ }^{122 b, 122 a}$ T. Kharlamova, ${ }^{122 b, 122 a}$ E. E. Khoda, ${ }^{175}$ A. Khodinov, ${ }^{166}$ T. J. Khoo, ${ }^{54}$ E. Khramov, ${ }^{80}$ J. Khubua, ${ }^{159 b}$ S. Kido,${ }^{83}$ M. Kiehn,${ }^{54}$ C. R. Kilby,${ }^{94}$ Y. K. Kim, ${ }^{37}$ N. Kimura, ${ }^{95}$ O. M. Kind, ${ }^{19}$ B. T. King, ${ }^{91, a}$ D. Kirchmeier ${ }^{48}$ J. Kirk, ${ }^{144}$ A. E. Kiryunin, ${ }^{115}$ T. Kishimoto, ${ }^{163}$ D. P. Kisliuk, ${ }^{167}$ V. Kitali, ${ }^{46}$ O. Kivernyk, ${ }^{5}$ T. Klapdor-Kleingrothaus, ${ }^{52}$ M. Klassen, ${ }^{61 \mathrm{a}}$ M. H. Klein, ${ }^{106}$ M. Klein, ${ }^{91}$ U. Klein, ${ }^{91}$ K. Kleinknecht, ${ }^{100}$ P. Klimek, ${ }^{121}$ A. Klimentov, ${ }^{29}$ T. Klingl,${ }^{24}$ T. Klioutchnikova, ${ }^{36}$ F. F. Klitzner, ${ }^{114}$ P. Kluit, ${ }^{120}$ S. Kluth, ${ }^{115}$ E. Kneringer, ${ }^{77}$ E. B. F. G. Knoops, ${ }^{102}$ A. Knue,${ }^{52}$ D. Kobayashi, ${ }^{88}$ T. Kobayashi, ${ }^{163}$ M. Kobel,${ }^{48}$ M. Kocian, ${ }^{153}$ P. Kodys, ${ }^{143}$ P. T. Koenig, ${ }^{24}$

T. Koffas, ${ }^{34}$ N. M. Köhler, ${ }^{36}$ T. Koi, ${ }^{153}$ M. Kolb, ${ }^{61 b}$ I. Koletsou, ${ }^{5}$ T. Komarek, ${ }^{131}$ T. Kondo, ${ }^{82}$ N. Kondrashova, ${ }^{60 c}$

K. Köneke, ${ }^{52}$ A. C. König, ${ }^{119}$ T. Kono, ${ }^{126}$ R. Konoplich, ${ }^{125, g g}$ V. Konstantinides, ${ }^{95}$ N. Konstantinidis, ${ }^{95}$ B. Konya, ${ }^{97}$ R. Kopeliansky, ${ }^{66}$ S. Koperny, ${ }^{84 a}$ K. Korcyl ${ }^{85}$ K. Kordas, ${ }^{162}$ G. Koren, ${ }^{161}$ A. Korn, ${ }^{95}$ I. Korolkov, ${ }^{14}$ E. V. Korolkova, ${ }^{149}$ N. Korotkova, ${ }^{113}$ O. Kortner, ${ }^{115}$ S. Kortner, ${ }^{115}$ T. Kosek, ${ }^{143}$ V. V. Kostyukhin, ${ }^{166}$ A. Kotsokechagia, ${ }^{65}$ A. Kotwal, ${ }^{49}$ A. Koulouris, ${ }^{10}$ A. Kourkoumeli-Charalampidi, ${ }^{71 \mathrm{a}, 71 \mathrm{~b}}$ C. Kourkoumelis, ${ }^{9}$ E. Kourlitis, ${ }^{149}$ V. Kouskoura, ${ }^{29}$

A. B. Kowalewska, ${ }^{85}$ R. Kowalewski, ${ }^{176}$ C. Kozakai, ${ }^{163}$ W. Kozanecki, ${ }^{145}$ A. S. Kozhin, ${ }^{123}$ V. A. Kramarenko, ${ }^{113}$ G. Kramberger, ${ }^{92}$ D. Krasnopevtsev, ${ }^{60 a}$ M. W. Krasny, ${ }^{136}$ A. Krasznahorkay, ${ }^{36}$ D. Krauss, ${ }^{115}$ J. A. Kremer, ${ }^{84 a}$

J. Kretzschmar, ${ }^{91}$ P. Krieger, ${ }^{167}$ F. Krieter ${ }^{114}$ A. Krishnan, ${ }^{61 b}$ K. Krizka, ${ }^{18}$ K. Kroeninger, ${ }^{47}$ H. Kroha, ${ }^{115}$ J. Kroll, ${ }^{141}$ J. Kroll, ${ }^{137}$ K. S. Krowpman, ${ }^{107}$ J. Krstic, ${ }^{16}$ U. Kruchonak, ${ }^{80}$ H. Krüger,${ }^{24}$ N. Krumnack, ${ }^{79}$ M. C. Kruse, ${ }^{49}$ J. A. Krzysiak, ${ }^{85}$ T. Kubota, ${ }^{105}$ O. Kuchinskaia, ${ }^{166}$ S. Kuday, ${ }^{4 b}$ J. T. Kuechler, ${ }^{46}$ S. Kuehn, ${ }^{36}$ A. Kugel, ${ }^{61 \mathrm{a}}$ T. Kuhl, ${ }^{46}$ V. Kukhtin, ${ }^{80}$ R. Kukla, ${ }^{102}$ Y. Kulchitsky, ${ }^{108, \text { hh }}$ S. Kuleshov, ${ }^{147 d}$ Y. P. Kulinich, ${ }^{173}$ M. Kuna, ${ }^{58}$ T. Kunigo ${ }^{86}$ A. Kupco, ${ }^{141}$ T. Kupfer, ${ }^{47}$ O. Kuprash,${ }^{52}$ H. Kurashige, ${ }^{83}$ L. L. Kurchaninov, ${ }^{168 a}$ Y. A. Kurochkin, ${ }^{108}$ A. Kurova, ${ }^{112}$ M. G. Kurth, ${ }^{15 a, 15 d}$ E. S. Kuwertz, ${ }^{36}$ M. Kuze, ${ }^{165}$ A. K. Kvam, ${ }^{148}$ J. Kvita, ${ }^{131}$ T. Kwan, ${ }^{104}$ A. La Rosa,${ }^{115}$ L. La Rotonda,${ }^{41 b, 41 a}$ F. La Ruffa, ${ }^{41 b, 41 a}$ C. Lacasta, ${ }^{174}$ F. Lacava, ${ }^{73 a, 73 b}$ D. P. J. Lack, ${ }^{101}$ H. Lacker, ${ }^{19}$ D. Lacour, ${ }^{136}$ E. Ladygin, ${ }^{80}$ R. Lafaye, ${ }^{5}$ B. Laforge, ${ }^{136}$ T. Lagouri, ${ }^{33 e}$ S. Lai, ${ }^{53}$ S. Lammers,${ }^{66}$

W. Lampl, ${ }^{7}$ C. Lampoudis, ${ }^{162}$ E. Lançon, ${ }^{29}$ U. Landgraf, ${ }^{52}$ M. P. J. Landon, ${ }^{93}$ M. C. Lanfermann, ${ }^{54}$ V. S. Lang ${ }^{46}$

J. C. Lange, ${ }^{53}$ R. J. Langenberg, ${ }^{36}$ A. J. Lankford, ${ }^{171}$ F. Lanni, ${ }^{29}$ K. Lantzsch, ${ }^{24}$ A. Lanza, ${ }^{71 a}$ A. Lapertosa, ${ }^{55 b, 55 a}$ S. Laplace, ${ }^{136}$ J. F. Laporte, ${ }^{145}$ T. Lari, ${ }^{69 a}$ F. Lasagni Manghi, ${ }^{23 b, 23 a}$ M. Lassnig, ${ }^{36}$ T. S. Lau, ${ }^{63 a}$ A. Laudrain, ${ }^{65}$ A. Laurier, ${ }^{34}$ M. Lavorgna, ${ }^{70 a, 70 b}$ S. D. Lawlor, ${ }^{94}$ M. Lazzaroni, ${ }^{69 a, 69 b}$ B. Le, ${ }^{105}$ E. Le Guirriec, ${ }^{102}$ M. LeBlanc, ${ }^{7}$ T. LeCompte, ${ }^{6}$ F. Ledroit-Guillon, ${ }^{58}$ A. C. A. Lee, ${ }^{95}$ C. A. Lee ${ }^{29}$ G. R. Lee, ${ }^{17}$ L. Lee, ${ }^{59}$ S. C. Lee, ${ }^{158}$ S. J. Lee, ${ }^{34}$ S. Lee,${ }^{79}$ B. Lefebvre, ${ }^{168 a}$ H. P. Lefebvre, ${ }^{94}$ M. Lefebvre, ${ }^{176}$ F. Legger, ${ }^{114}$ C. Leggett, ${ }^{18}$ K. Lehmann, ${ }^{152}$ N. Lehmann, ${ }^{182}$ G. Lehmann Miotto, ${ }^{36}$ W. A. Leight, ${ }^{46}$ A. Leisos, ${ }^{162, \text { ii }}$ M. A. L. Leite,${ }^{81 \mathrm{~d}}$ C. E. Leitgeb, ${ }^{114}$ R. Leitner, ${ }^{143}$ D. Lellouch, ${ }^{180, \mathrm{a}}$ K. J. C. Leney, ${ }^{42}$ T. Lenz, ${ }^{24}$ B. Lenzi,${ }^{36}$ R. Leone, ${ }^{7}$ S. Leone, ${ }^{72 a}$ C. Leonidopoulos, ${ }^{50}$ A. Leopold, ${ }^{136}$ G. Lerner,${ }^{156}$ C. Leroy, ${ }^{110}$ R. Les,${ }^{167}$ C. G. Lester,${ }^{32}$ M. Levchenko, ${ }^{138}$ J. Levêque, ${ }^{5}$ D. Levin, ${ }^{106}$ L. J. Levinson, ${ }^{180}$ D. J. Lewis, ${ }^{21}$ B. Li ${ }^{15 b}$ B. Li, ${ }^{106} \mathrm{C}-\mathrm{Q} . \mathrm{Li},{ }^{60 \mathrm{a}} \mathrm{F}$. Li $,{ }^{60 \mathrm{c}} \mathrm{H} . \mathrm{Li},{ }^{60 \mathrm{a}}$ H. Li, ${ }^{60 \mathrm{~b}}$ J. Li ${ }^{60 \mathrm{c}}$ K. Li, ${ }^{153} \mathrm{~L} . \mathrm{Li},{ }^{60 \mathrm{c}} \mathrm{M} . \mathrm{Li},{ }^{15 \mathrm{a}, 15 \mathrm{~d}}$ Q. Li, ${ }^{15,15 \mathrm{~d}}$ Q. Y. Li, ${ }^{60 \mathrm{a}} \mathrm{S} . \mathrm{Li},{ }^{60 \mathrm{~d}, 60 \mathrm{c}} \mathrm{X} . \mathrm{Li}^{6}{ }^{46} \mathrm{Y} . \mathrm{Li},{ }^{46} \mathrm{Z} . \mathrm{Li},{ }^{60 \mathrm{~b}} \mathrm{Z} . \mathrm{Liang},{ }^{15 \mathrm{a}}$ B. Liberti, ${ }^{74 a}$ A. Liblong, ${ }^{167}$ K. Lie,${ }^{6 c}$ C. Y. Lin, ${ }^{32}$ K. Lin, ${ }^{107}$ T. H. Lin, ${ }^{100}$ R. A. Linck, ${ }^{66}$ J. H. Lindon, ${ }^{21}$ A. L. Lionti, ${ }^{54}$ E. Lipeles, ${ }^{137}$ A. Lipniacka, ${ }^{17}$ M. Lisovyi ${ }^{61 b}$ T. M. Liss, ${ }^{173, j j}$ A. Lister, ${ }^{175}$ A. M. Litke, ${ }^{146}$ J. D. Little, ${ }^{8}$ B. Liu, ${ }^{79}$ B. L. Liu, ${ }^{6}$ H. B. Liu ${ }^{29}$ H. Liu, ${ }^{106}$ J. B. Liu, ${ }^{60 a}$ J. K. K. Liu ${ }^{135}$ K. Liu,${ }^{136}$ M. Liu, ${ }^{60 a}$ P. Liu, ${ }^{18}$ Y. Liu, ${ }^{15 a, 15 d}$ Y. L. Liu,${ }^{106}$ Y. W. Liu, ${ }^{60 a}$ M. Livan, ${ }^{71 a, 71 b}$ A. Lleres, ${ }^{58}$ J. Llorente Merino, ${ }^{152}$ S. L. Lloyd,${ }^{93}$ C. Y. Lo, ${ }^{63 b}$ F. Lo Sterzo, ${ }^{42}$ E. M. Lobodzinska, ${ }^{46}$ P. Loch, ${ }^{7}$ S. Loffredo, ${ }^{74 a, 74 b}$ T. Lohse, ${ }^{19}$ K. Lohwasser, ${ }^{149}$ M. Lokajicek, ${ }^{141}$ J. D. Long, ${ }^{173}$ R. E. Long, ${ }_{5}^{90}$ L. Longo, ${ }^{36}$ K. A. Looper, ${ }^{127}$ J. A. Lopez ${ }^{147 d}$ I. Lopez Paz, ${ }^{101}$ A. Lopez Solis, ${ }^{149}$ J. Lorenz, ${ }^{114}$ N. Lorenzo Martinez, ${ }^{5}$ M. Losada, ${ }^{22 a}$ P. J. Lösel, ${ }^{114}$

A. Lösle ${ }^{52}$ X. Lou, ${ }^{46}$ X. Lou, ${ }^{15 a}$ A. Lounis, ${ }^{65}$ J. Love, ${ }^{6}$ P. A. Love, ${ }^{90}$ J. J. Lozano Bahilo, ${ }^{174}$ M. Lu, ${ }^{60 a}$ Y. J. Lu, ${ }^{64}$ H. J. Lubatti, ${ }^{148}$ C. Luci, ${ }^{73 a}, 73 \mathrm{~b}$ A. Lucotte,${ }^{58}$ C. Luedtke, ${ }^{52}$ F. Luehring ${ }^{66}{ }^{6}$ I. Luise, ${ }^{136}$ L. Luminari ${ }^{73 a}$ B. Lund-Jensen, ${ }^{154}$ M. S. Lutz, ${ }^{103}$ D. Lynn, ${ }^{29}$ R. Lysak, ${ }^{141}$ E. Lytken, ${ }^{97}$ F. Lyu, ${ }^{15 a}$ V. Lyubushkin, ${ }^{80}$ T. Lyubushkina,${ }^{80}$ H. Ma,${ }^{29}$ L. L. Ma, ${ }^{60 b}$ 
Y. Ma, ${ }^{60 b}$ G. Maccarrone,${ }^{51}$ A. Macchiolo, ${ }^{115}$ C. M. Macdonald, ${ }^{149}$ J. Machado Miguens, ${ }^{137}$ D. Madaffari, ${ }^{174}$ R. Madar, ${ }^{38}$ W. F. Mader, ${ }^{48}$ N. Madysa, ${ }^{48}$ J. Maeda, ${ }^{83}$ S. Maeland, ${ }^{17}$ T. Maeno, ${ }^{29}$ M. Maerker, ${ }^{48}$ A. S. Maevskiy, ${ }^{113}$ V. Magerl, ${ }^{52}$ N. Magini, ${ }^{79}$ D. J. Mahon, ${ }^{39}$ C. Maidantchik, ${ }^{81 b}$ T. Maier, ${ }^{114}$ A. Maio, ${ }^{140 a, 140 b, 140 \mathrm{~d}}$ K. Maj, ${ }^{84 \mathrm{a}}$ O. Majersky, ${ }^{28 \mathrm{a}}$ S. Majewski, ${ }^{132}$ Y. Makida ${ }^{82}$ N. Makovec, ${ }^{65}$ B. Malaescu, ${ }^{136}$ Pa. Malecki, ${ }^{85}$ V. P. Maleev, ${ }^{138}$ F. Malek ${ }^{58}$ U. Mallik,${ }^{78}$ D. Malon, ${ }^{6}$ C. Malone, ${ }^{32}$ S. Maltezos, ${ }^{10}$ S. Malyukov, ${ }^{80}$ J. Mamuzic, ${ }^{174}$ G. Mancini,${ }^{51}$ I. Mandic,${ }^{92}$ L. Manhaes de Andrade Filho, ${ }^{81 a}$ I. M. Maniatis, ${ }^{162}$ J. Manjarres Ramos, ${ }^{48}$ K. H. Mankinen, ${ }^{97}$ A. Mann, ${ }^{114}$ A. Manousos, ${ }^{77}$ B. Mansoulie, ${ }^{145}$ I. Manthos, ${ }^{162}$ S. Manzoni, ${ }^{120}$ A. Marantis, ${ }^{162}$ G. Marceca, ${ }^{30}$ L. Marchese,${ }^{135}$ G. Marchiori, ${ }^{136}$ M. Marcisovsky ${ }^{141}$ L. Marcoccia,${ }^{74 a, 74 b}$ C. Marcon, ${ }^{97}$ C. A. Marin Tobon, ${ }^{36}$ M. Marjanovic, ${ }^{129}$ Z. Marshall, ${ }^{18}$ M. U. F. Martensson, ${ }^{172}$ S. Marti-Garcia, ${ }^{174}$ C. B. Martin, ${ }^{127}$ T. A. Martin, ${ }^{178}$ V. J. Martin, ${ }^{50}$ B. Martin dit Latour, ${ }^{17}$ L. Martinelli, ${ }^{75,75 b}$ M. Martinez, ${ }^{14, w}$ V. I. Martinez Outschoorn, ${ }^{103}$ S. Martin-Haugh, ${ }^{144}$ V. S. Martoiu, ${ }^{27 b}$ A. C. Martyniuk, ${ }^{95}$ A. Marzin, ${ }^{36}$ S. R. Maschek, ${ }^{115}$ L. Masetti, ${ }^{100}$ T. Mashimo, ${ }^{163}$ R. Mashinistov, ${ }^{111}$ J. Masik, ${ }^{101}$ A. L. Maslennikov, ${ }^{122 b, 122 a}$ L. Massa, ${ }^{74 a, 74 b}$ P. Massarotti ${ }^{70 a, 70 b}$ P. Mastrandrea, ${ }^{72 a, 72 b}$ A. Mastroberardino, ${ }^{41 b, 41 \mathrm{a}}$ T. Masubuchi, ${ }^{163}$ D. Matakias, ${ }^{10}$ A. Matic, ${ }^{114}$ P. Mättig, ${ }^{24}$ J. Maurer, ${ }^{27 b}$ B. Maček, ${ }^{92}$ D. A. Maximov, ${ }^{122 b, 122 a}$ R. Mazini, ${ }^{158}$ I. Maznas, ${ }^{162}$ S. M. Mazza, ${ }^{146}$ S. P. Mc Kee, ${ }^{106}$ T. G. McCarthy, ${ }^{115}$ W. P. McCormack, ${ }^{18}$ E. F. McDonald, ${ }^{105}$ J. A. Mcfayden, ${ }^{36}$ G. Mchedlidze, ${ }^{159 b}$ M. A. McKay, ${ }^{42}$ K. D. McLean, ${ }^{176}$ S. J. McMahon, ${ }^{144}$ P. C. McNamara, ${ }^{105}$ C. J. McNicol, ${ }^{178}$ R. A. McPherson, ${ }^{176, p}$ J. E. Mdhluli, ${ }^{33 e}$ Z. A. Meadows, ${ }^{103}$ S. Meehan, ${ }^{36}$ T. Megy, ${ }^{52}$ S. Mehlhase, ${ }^{114}$ A. Mehta, ${ }^{91}$ T. Meideck, ${ }^{58}$ B. Meirose,${ }^{43}$ D. Melini, ${ }^{174}$ B. R. Mellado Garcia, ${ }^{33 e}$ J. D. Mellenthin ${ }^{53}$ M. Melo, ${ }^{28 a}$ F. Meloni, ${ }^{46}$ A. Melzer, ${ }^{24}$ S. B. Menary, ${ }^{101}$ E. D. Mendes Gouveia, ${ }^{140 a, 140 e}$ L. Meng, ${ }^{36}$ X. T. Meng, ${ }^{106}$ S. Menke, ${ }^{115}$ E. Meoni, ${ }^{41 \mathrm{~b}, 41 \mathrm{a}}$ S. Mergelmeyer, ${ }^{19}$ S. A. M. Merkt, ${ }^{139}$ C. Merlassino, ${ }^{20}$ P. Mermod, ${ }^{54}$ L. Merola ${ }^{70 a, 70 b}$ C. Meroni, ${ }^{69 a}$ O. Meshkov, ${ }^{113,111}$ J. K. R. Meshreki, ${ }^{151}$ A. Messina, ${ }^{73 a, 73 b}$ J. Metcalfe, ${ }^{6}$ A. S. Mete, ${ }^{171}$ C. Meyer, ${ }^{66}$ J. Meyer, ${ }^{160}$ J-P. Meyer, ${ }^{145}$ H. Meyer Zu Theenhausen, ${ }^{61 a}$ F. Miano, ${ }^{156}$ M. Michetti, ${ }^{19}$ R. P. Middleton, ${ }^{144}$ L. Mijović, ${ }^{50}$ G. Mikenberg, ${ }^{180}$ M. Mikestikova, ${ }^{141}$ M. Mikuž,${ }^{92}$ H. Mildner, ${ }^{149}$ M. Milesi, ${ }^{105}$ A. Milic, ${ }^{167}$ D. A. Millar, ${ }^{93}$ D. W. Miller, ${ }^{37}$ A. Milov, ${ }^{180}$ D. A. Milstead, ${ }^{45 a, 45 b}$ R. A. Mina, ${ }^{153, f f}$ A. A. Minaenko, ${ }^{123}$ M. Miñano Moya, ${ }^{174}$ I. A. Minashvili, ${ }^{159 b}$ A. I. Mincer, ${ }^{125}$ B. Mindur, ${ }^{84 a}$ M. Mineev,${ }^{80}$ Y. Minegishi, ${ }^{163}$ L. M. Mir, ${ }^{14}$ A. Mirto, ${ }^{68 a, 68 b}$ K. P. Mistry, ${ }^{137}$ T. Mitani, ${ }^{179}$ J. Mitrevski, ${ }^{114}$ V. A. Mitsou, ${ }^{174}$ M. Mittal,${ }^{60 c}$ O. Miu, ${ }^{167}$ A. Miucci, ${ }^{20}$ P. S. Miyagawa, ${ }^{149}$ A. Mizukami, ${ }^{82}$ J. U. Mjörnmark, ${ }^{97}$ T. Mkrtchyan, ${ }^{184}$ M. Mlynarikova, ${ }^{143}$ T. Moa ${ }^{45 a, 45 b}$ K. Mochizuki, ${ }^{110}$ P. Mogg ${ }^{52}$ S. Mohapatra, ${ }^{39}$ R. Moles-Valls, ${ }^{24}$ M. C. Mondragon, ${ }^{107}$ K. Mönig, ${ }^{46}$ J. Monk, ${ }^{40}$ E. Monnier, ${ }^{102}$ A. Montalbano, ${ }^{152}$ J. Montejo Berlingen, ${ }^{36}$ M. Montella, ${ }^{95}$ F. Monticelli, ${ }^{89}$ S. Monzani, ${ }^{69 a}$ N. Morange, ${ }^{65}$ D. Moreno, ${ }^{22 a}$ M. Moreno Llácer ${ }^{36}$ C. Moreno Martinez, ${ }^{14}$ P. Morettini, ${ }^{55 b}$ M. Morgenstern, ${ }^{120}$ S. Morgenstern,${ }^{48}$ D. Mori, ${ }^{152}$ M. Morii,${ }^{59}$ M. Morinaga, ${ }^{179}$ V. Morisbak, ${ }^{134}$ A. K. Morley, ${ }^{36}$ G. Mornacchi, ${ }^{36}$ A. P. Morris, ${ }^{95}$ L. Morvaj, ${ }^{155}$ P. Moschovakos,${ }^{36}$ B. Moser, ${ }^{120}$ M. Mosidze,${ }^{159 b}$ T. Moskalets, ${ }^{145}$ H. J. Moss, ${ }^{149}$ J. Moss, ${ }^{31, k k}$ E. J. W. Moyse, ${ }^{103}$ S. Muanza, ${ }^{102}$ J. Mueller, ${ }^{139}$ R. S. P. Mueller, ${ }^{114}$ D. Muenstermann, ${ }^{90}$ G. A. Mullier, ${ }^{97}$ J. L. Munoz Martinez,${ }^{14}$ F. J. Munoz Sanchez, ${ }^{101}$ P. Murin, ${ }^{28 b}$ W. J. Murray ${ }^{178,144}$ A. Murrone, ${ }^{69 a, 69 b}$ M. Muškinja, ${ }^{18}$ C. Mwewa, ${ }^{33 a}$ A. G. Myagkov, ${ }^{123, k}$ J. Myers,${ }^{132}$ M. Myska, ${ }^{142}$ B. P. Nachman, ${ }^{18}$ O. Nackenhorst,${ }^{47}$ A. Nag Nag, ${ }^{48}$ K. Nagai, ${ }^{135}$ K. Nagano,${ }^{82}$ Y. Nagasaka, ${ }^{62}$ M. Nagel,${ }^{52}$ J. L. Nagle,${ }^{29}$ E. Nagy, ${ }^{102}$ A. M. Nairz,${ }^{36}$ Y. Nakahama,${ }^{117}$ K. Nakamura, ${ }^{82}$ T. Nakamura, ${ }^{163}$ I. Nakano, ${ }^{128}$ H. Nanjo, ${ }^{133}$ F. Napolitano, ${ }^{61 a}$ R. F. Naranjo Garcia, ${ }^{46}$ R. Narayan, ${ }^{42}$ I. Naryshkin, ${ }^{138}$ T. Naumann, ${ }^{46}$ G. Navarro, ${ }^{22 a}$ P. Y. Nechaeva, ${ }^{111}$ F. Nechansky, ${ }^{46}$ T. J. Neep, ${ }^{21}$ A. Negri, ${ }^{71 a, 71 b}$ M. Negrini, ${ }^{23 b}$ C. Nellist, ${ }^{53}$ M. E. Nelson, ${ }^{135}$ S. Nemecek, ${ }^{141}$ P. Nemethy, ${ }^{125}$ M. Nessi ${ }^{36,11}$ M. S. Neubauer, ${ }^{173}$ M. Neumann, ${ }^{182}$ P. R. Newman, ${ }^{21}$ Y. S. Ng, ${ }^{19}$ Y. W. Y. Ng, ${ }^{171}$ B. Ngair, ${ }^{35 e}$ H. D. N. Nguyen, ${ }^{102}$ T. Nguyen Manh, ${ }^{110}$ E. Nibigira ${ }^{38}$ R. B. Nickerson, ${ }^{135}$ R. Nicolaidou, ${ }^{145}$ D. S. Nielsen, ${ }^{40}$ J. Nielsen, ${ }^{146}$ N. Nikiforou, ${ }^{11}$ V. Nikolaenko, ${ }^{123, \mathrm{k}}$ I. Nikolic-Audit, ${ }^{136}$ K. Nikolopoulos, ${ }^{21}$ P. Nilsson, ${ }^{29}$ H. R. Nindhito, ${ }^{54}$ Y. Ninomiya,${ }^{82}$ A. Nisati, ${ }^{73 a}$ N. Nishu, ${ }^{60 \mathrm{c}}$ R. Nisius, ${ }^{115}$ I. Nitsche, ${ }^{47}$ T. Nitta, ${ }^{179}$ T. Nobe, ${ }^{163}$ Y. Noguchi, ${ }^{86}$ I. Nomidis, ${ }^{136}$ M. A. Nomura ${ }^{29}$ M. Nordberg, ${ }^{36}$ N. Norjoharuddeen, ${ }^{135}$ T. Novak, ${ }^{92}$ O. Novgorodova, ${ }^{48}$ R. Novotny, ${ }^{142}$ L. Nozka, ${ }^{131}$ K. Ntekas, ${ }^{171}$ E. Nurse, ${ }^{95}$ F. G. Oakham, ${ }^{34, d}$ H. Oberlack, ${ }^{115}$ J. Ocariz, ${ }^{136}$ A. Ochi ${ }^{83}$ I. Ochoa,${ }^{39}$ J. P. Ochoa-Ricoux, ${ }^{147 a}$ K. O'Connor, ${ }^{26}$ S. Oda, ${ }^{88}$ S. Odaka, ${ }^{82}$ S. Oerdek ${ }^{53}$ A. Ogrodnik, ${ }^{84 a}$ A. Oh, ${ }^{101}$ S. H. Oh, ${ }^{49}$ C. C. Ohm, ${ }^{154}$ H. Oide, ${ }^{165}$ M. L. Ojeda,${ }^{167}$ H. Okawa, ${ }^{169}$ Y. Okazaki, ${ }^{86}$ Y. Okumura, ${ }^{163}$ T. Okuyama, ${ }^{82}$ A. Olariu, ${ }^{27 b}$ L. F. Oleiro Seabra, ${ }^{14 a}$ S. A. Olivares Pino, ${ }^{147 a}$

D. Oliveira Damazio, ${ }^{29}$ J. L. Oliver, ${ }^{1}$ M. J. R. Olsson, ${ }^{171}$ A. Olszewski, ${ }^{85}$ J. Olszowska, ${ }^{85}$ D. C. O’Neil, ${ }^{152}$ A. P. O'neill, ${ }^{135}$ A. Onofre, ${ }^{140 a, 140 e}$ P. U. E. Onyisi, ${ }^{11}$ H. Oppen, ${ }^{134}$ M. J. Oreglia, ${ }^{37}$ G. E. Orellana, ${ }^{89}$ D. Orestano, ${ }^{75 a, 75 b}$ N. Orlando, ${ }^{14}$ R. S. Orr, ${ }^{167}$ V. O'Shea,${ }^{57}$ R. Ospanov, ${ }^{60 a}$ G. Otero y Garzon, ${ }^{30}$ H. Otono, ${ }^{88}$ P. S. Ott, ${ }^{61 a}$ M. Ouchrif, ${ }^{35 \mathrm{~d}}$ J. Ouellette, ${ }^{29}$ F. Ould-Saada, ${ }^{134}$ A. Ouraou, ${ }^{145}$ Q. Ouyang, ${ }^{15 a}$ M. Owen, ${ }^{57}$ R. E. Owen, ${ }^{21}$ V. E. Ozcan, ${ }^{12 c}$ N. Ozturk,${ }^{8}$ J. Pacalt, ${ }^{131}$ H. A. Pacey, ${ }^{32}$ K. Pachal, ${ }^{49}$ A. Pacheco Pages, ${ }^{14}$ C. Padilla Aranda, ${ }^{14}$ S. Pagan Griso, ${ }^{18}$ M. Paganini, ${ }^{183}$ G. Palacino, ${ }^{66}$ 
S. Palazzo, ${ }^{50}$ S. Palestini, ${ }^{36}$ M. Palka ${ }^{84 b}$ D. Pallin, ${ }^{38}$ I. Panagoulias, ${ }^{10}$ C. E. Pandini, ${ }^{36}$ J. G. Panduro Vazquez, ${ }^{94}$ P. Pani, ${ }^{46}$ G. Panizzo, ${ }^{67 a, 67 \mathrm{c}}$ L. Paolozzi,${ }^{54}$ C. Papadatos, ${ }^{110}$ K. Papageorgiou, ${ }^{9, u}$ S. Parajuli, ${ }^{43}$ A. Paramonov, ${ }^{6}$

D. Paredes Hernandez, ${ }^{63 b}$ S. R. Paredes Saenz, ${ }^{135}$ B. Parida, ${ }^{166}$ T. H. Park, ${ }^{167}$ A. J. Parker, ${ }^{31}$ M. A. Parker, ${ }^{32}$ F. Parodi, ${ }^{55 b, 55 a}$ E. W. Parrish, ${ }^{121}$ J. A. Parsons, ${ }^{39}$ U. Parzefall, ${ }^{52}$ L. Pascual Dominguez, ${ }^{136}$ V. R. Pascuzzi, ${ }^{167}$ J. M. P. Pasner, ${ }^{146}$

F. Pasquali, ${ }^{120}$ E. Pasqualucci, ${ }^{73 a}$ S. Passaggio, ${ }^{55 b}$ F. Pastore,${ }^{94}$ P. Pasuwan, ${ }^{45 a, 45 b}$ S. Pataraia, ${ }^{100}$ J. R. Pater,${ }^{101}$ A. Pathak,${ }^{181, e}$ T. Pauly, ${ }^{36}$ B. Pearson, ${ }^{115}$ M. Pedersen, ${ }^{134}$ L. Pedraza Diaz, ${ }^{119}$ R. Pedro, ${ }^{14 a}$ T. Peiffer, ${ }^{53}$ S. V. Peleganchuk, ${ }^{122 b, 122 a}$ O. Penc, ${ }^{141}$ H. Peng, ${ }^{60 a}$ B. S. Peralva, ${ }^{81 a}$ M. M. Perego, ${ }^{65}$ A. P. Pereira Peixoto, ${ }^{140 a}$ D. V. Perepelitsa, ${ }^{29}$ F. Peri, ${ }^{19}$ L. Perini, ${ }^{69 a, 69 b}$ H. Pernegger, ${ }^{36}$ S. Perrella, ${ }^{70 a, 70 b}$ K. Peters, ${ }^{46}$ R. F. Y. Peters, ${ }^{101}$ B. A. Petersen, ${ }^{36}$ T. C. Petersen, ${ }^{40}$ E. Petit, ${ }^{102}$ A. Petridis, ${ }^{1}$ C. Petridou, ${ }^{162}$ P. Petroff, ${ }^{65}$ M. Petrov,${ }^{135}$ F. Petrucci, ${ }^{75 a}, 75 b$ M. Pettee,${ }^{183}$ N. E. Pettersson, ${ }^{103}$ K. Petukhova, ${ }^{143}$ A. Peyaud, ${ }^{145}$ R. Pezoa, ${ }^{147 d}$ L. Pezzotti, ${ }^{71 a, 71 b}$ T. Pham, ${ }^{105}$ F. H. Phillips,${ }^{107}$ P. W. Phillips,${ }^{144}$ M. W. Phipps,${ }^{173}$ G. Piacquadio, ${ }^{155}$ E. Pianori, ${ }^{18}$ A. Picazio, ${ }^{103}$ R. H. Pickles, ${ }^{101}$ R. Piegaia, ${ }^{30}$ D. Pietreanu, ${ }^{27 b}$ J. E. Pilcher, ${ }^{37}$ A. D. Pilkington, ${ }^{101}$ M. Pinamonti, ${ }^{74 a, 74 b}$ J. L. Pinfold, ${ }^{3}$ M. Pitt, ${ }^{161}$ L. Pizzimento, ${ }^{74 a, 74 b}$ M.-A. Pleier, ${ }^{29}$ V. Pleskot,${ }^{143}$ E. Plotnikova, ${ }^{80}$ P. Podberezko, ${ }^{122 b, 122 a}$ R. Poettgen,${ }^{97}$ R. Poggi, ${ }^{54}$ L. Poggioli, ${ }^{65}$ I. Pogrebnyak, ${ }^{107}$ D. Pohl,${ }^{24}$ I. Pokharel, ${ }^{53}$ G. Polesello, ${ }^{71 \mathrm{a}}$ A. Poley, ${ }^{18}$ A. Policicchio $,{ }^{73 a}, 73 \mathrm{~b}$ R. Polifka, ${ }^{143}$ A. Polini, ${ }^{23 b}$ C. S. Pollard, ${ }^{46}$ V. Polychronakos, ${ }^{29}$ D. Ponomarenko, ${ }^{112}$ L. Pontecorvo, ${ }^{36}$ S. Popa, ${ }^{27 a}$ G. A. Popeneciu, ${ }^{27 d}$ L. Portales, ${ }^{5}$ D. M. Portillo Quintero, ${ }^{58}$ S. Pospisil, ${ }^{142}$ K. Potamianos, ${ }^{46}$ I. N. Potrap, ${ }^{80}$ C. J. Potter, ${ }^{32}$ H. Potti, ${ }^{11}$ T. Poulsen, ${ }^{97}$ J. Poveda, ${ }^{36}$ T. D. Powell, ${ }^{149}$ G. Pownall, ${ }^{46}$ M. E. Pozo Astigarraga, ${ }^{36}$ P. Pralavorio, ${ }^{102}$ S. Prell, ${ }^{79}$ D. Price, ${ }^{101}$ M. Primavera, ${ }^{68 a}$ S. Prince,${ }^{104}$ M. L. Proffitt, ${ }^{148}$ N. Proklova, ${ }^{112}$ K. Prokofiev, ${ }^{63 \mathrm{c}}$ F. Prokoshin, ${ }^{80} \mathrm{~S}$. Protopopescu, ${ }^{29}$ J. Proudfoot, ${ }^{6}$ M. Przybycien, ${ }^{84 a}$ D. Pudzha, ${ }^{138}$ A. Puri, ${ }^{173}$ P. Puzo, ${ }^{65}$ J. Qian, ${ }^{106}$ Y. Qin, ${ }^{101}$ A. Quadt, ${ }^{53}$ M. Queitsch-Maitland ${ }^{46}$ A. Qureshi, ${ }^{1}$ M. Racko, ${ }^{28 a}$ P. Rados, ${ }^{105}$ F. Ragusa, ${ }^{69 a, 69 b}$ G. Rahal,${ }^{98}$ J. A. Raine,${ }^{54}$ S. Rajagopalan, ${ }^{29}$ A. Ramirez Morales,${ }^{93}$ K. Ran, ${ }^{15 a, 15 \mathrm{~d}}$ T. Rashid, ${ }^{65}$ S. Raspopov, ${ }^{5}$ D. M. Rauch, ${ }^{46}$ F. Rauscher, ${ }^{114}$ S. Rave, ${ }^{100}$ B. Ravina ${ }^{149}$ I. Ravinovich, ${ }^{180}$ J. H. Rawling, ${ }^{101}$ M. Raymond, ${ }^{36}$ A. L. Read, ${ }^{134}$ N. P. Readioff,${ }^{58}$ M. Reale,${ }^{68 a, 68 b}$ D. M. Rebuzzi, ${ }^{71 \mathrm{a}, 71 \mathrm{~b}}$ A. Redelbach, ${ }^{177}$ G. Redlinger, ${ }^{29}$ K. Reeves,${ }^{43}$ L. Rehnisch, ${ }^{19}$ J. Reichert, ${ }^{137}$ D. Reikher, ${ }^{161}$ A. Reiss ${ }^{100}$ A. Rej ${ }^{151}$ C. Rembser,${ }^{36}$ M. Renda,${ }^{27 b}$ M. Rescigno, ${ }^{73 a}$ S. Resconi, ${ }^{69 a}$ E. D. Resseguie, ${ }^{137}$ S. Rettie, ${ }^{175}$ E. Reynolds ${ }^{21}$ O. L. Rezanova, ${ }^{122 b, 122 \mathrm{a}}$ P. Reznicek, ${ }^{143}$ E. Ricci ${ }^{76 a, 76 \mathrm{~b}}$ R. Richter, ${ }^{115}$ S. Richter, ${ }^{46}$ E. Richter-Was, ${ }^{84 b}$ O. Ricken, ${ }^{24}$ M. Ridel, ${ }^{136}$ P. Rieck,${ }^{115}$ C. J. Riegel,${ }^{182}$ O. Rifki, ${ }^{46}$ M. Rijssenbeek, ${ }^{155}$ A. Rimoldi, ${ }^{7 \mathrm{la}, 71 \mathrm{~b}}$ M. Rimoldi, ${ }^{46}$ L. Rinaldi, ${ }^{23 \mathrm{~b}}$ G. Ripellino, ${ }^{154}$ I. Riu, ${ }^{14}$ J. C. Rivera Vergara, ${ }^{176}$ F. Rizatdinova, ${ }^{130}$ E. Rizvi, ${ }^{93}$ C. Rizzi, ${ }^{36}$ R. T. Roberts, ${ }^{101}$ S. H. Robertson, ${ }^{104, p}$ M. Robin, ${ }^{46}$ D. Robinson, ${ }^{32}$ J. E. M. Robinson, ${ }^{46}$

C. M. Robles Gajardo, ${ }^{147 \mathrm{~d}}$ A. Robson, ${ }^{57}$ A. Rocchi, ${ }^{74 a, 74 \mathrm{~b}}$ E. Rocco, ${ }^{100}$ C. Roda,${ }^{72 \mathrm{a}, 72 \mathrm{~b}}$ S. Rodriguez Bosca, ${ }^{174}$ A. Rodriguez Perez, ${ }^{14}$ D. Rodriguez Rodriguez, ${ }^{174}$ A. M. Rodríguez Vera, ${ }^{168 \mathrm{~b}}$ S. Roe, ${ }^{36}$ O. Røhne, ${ }^{134}$ R. Röhrig, ${ }^{115}$ R. A. Rojas, ${ }^{147 d}$ C. P. A. Roland, ${ }^{66}$ J. Roloff, ${ }^{59}$ A. Romaniouk, ${ }^{112}$ M. Romano, ${ }^{23 b, 23 a}$ N. Rompotis, ${ }^{91}$ M. Ronzani, ${ }^{125}$ L. Roos, ${ }^{136}$ S. Rosati, ${ }^{73 a}$ K. Rosbach, ${ }^{52}$ G. Rosin, ${ }^{103}$ B. J. Rosser, ${ }^{137}$ E. Rossi, ${ }^{46}$ E. Rossi, ${ }^{75 a}, 75 b$ E. Rossi, ${ }^{70 a, 70 b}$ L. P. Rossi, ${ }^{55 b}$ L. Rossini ${ }^{69 \mathrm{a}, 69 \mathrm{~b}}$ R. Rosten, ${ }^{14}$ M. Rotaru, ${ }^{27 \mathrm{~b}}$ J. Rothberg, ${ }^{148}$ D. Rousseau, ${ }^{65}$ G. Rovelli, ${ }^{71 \mathrm{a}, 71 \mathrm{~b}}$ A. Roy, ${ }^{11}$ D. Roy, ${ }^{33 \mathrm{e}}$ A. Rozanov, ${ }^{102}$ Y. Rozen, ${ }^{160}$ X. Ruan, ${ }^{33 e}$ F. Rubbo, ${ }^{153}$ F. Rühr,${ }^{52}$ A. Ruiz-Martinez, ${ }^{174}$ A. Rummler, ${ }^{36}$ Z. Rurikova, ${ }^{52}$ N. A. Rusakovich ${ }^{80}$ H. L. Russell, ${ }^{104}$ L. Rustige, ${ }^{38,47}$ J. P. Rutherfoord, ${ }^{7}$ E. M. Rüttinger, ${ }^{149}$ M. Rybar, ${ }^{39}$ G. Rybkin, ${ }^{65}$ E. B. Rye,${ }^{134}$ A. Ryzhov, ${ }^{123}$ P. Sabatini,${ }^{53}$ G. Sabato, ${ }^{120}$ S. Sacerdoti, ${ }^{65}$ H. F-W. Sadrozinski, ${ }^{146}$ R. Sadykov, ${ }^{80}$ F. Safai Tehrani, ${ }^{73 a}$ B. Safarzadeh Samani, ${ }^{156}$ P. Saha, ${ }^{121}$ S. Saha,${ }^{104}$ M. Sahinsoy, ${ }^{61 a}$ A. Sahu, ${ }^{182}$ M. Saimpert, ${ }^{46}$ M. Saito, ${ }^{163}$ T. Saito, ${ }^{163}$ H. Sakamoto, ${ }^{163}$ A. Sakharov ${ }^{125, g g}$ D. Salamani,${ }^{54}$ G. Salamanna, ${ }^{75 a, 75 b}$ J. E. Salazar Loyola, ${ }^{147 d}$ P. H. Sales De Bruin, ${ }^{172}$ A. Salnikov, ${ }^{153}$ J. Salt, ${ }^{174}$ D. Salvatore ${ }^{41 b, 41 a}$ F. Salvatore, ${ }^{156}$ A. Salvucci, ${ }^{63 a, 63 b, 63 c}$ A. Salzburger, ${ }^{36}$ J. Samarati, ${ }^{36}$ D. Sammel, ${ }^{52}$ D. Sampsonidis, ${ }^{162}$ D. Sampsonidou, ${ }^{162}$ J. Sánchez,${ }^{174}$ A. Sanchez Pineda, ${ }^{67 a, 67 c}$ H. Sandaker, ${ }^{134}$ C. O. Sander, ${ }^{46}$ I. G. Sanderswood, ${ }^{90}$ M. Sandhoff, ${ }^{182}$ C. Sandoval, ${ }^{22 a}$ D. P. C. Sankey, ${ }^{144}$ M. Sannino,${ }^{55 b, 55 a}$ Y. Sano, ${ }^{117}$ A. Sansoni, ${ }^{51}$ C. Santoni, ${ }^{38}$ H. Santos, ${ }^{140 a, 140 b}$ S. N. Santpur, ${ }^{18}$ A. Santra, ${ }^{174}$ A. Sapronov,${ }^{80}$ J. G. Saraiva, ${ }^{140 a, 140 d}$ O. Sasaki, ${ }^{82}$ K. Sato, ${ }^{169}$ F. Sauerburger, ${ }^{52}$ E. Sauvan, ${ }^{5}$ P. Savard,${ }^{167, \mathrm{~d}}$ N. Savic, ${ }^{115}$ R. Sawada, ${ }^{163}$ C. Sawyer, ${ }^{144}$ L. Sawyer,${ }^{96, \mathrm{~mm}}$ C. Sbarra, ${ }^{23 b}$ A. Sbrizzi, ${ }^{23 a}$ T. Scanlon, ${ }^{95}$ J. Schaarschmidt, ${ }^{148}$ P. Schacht, ${ }^{115}$ B. M. Schachtner, ${ }^{114}$ D. Schaefer, ${ }^{37}$ L. Schaefer, ${ }^{137}$ J. Schaeffer, ${ }^{100}$ S. Schaepe ${ }^{36}$ U. Schäfer, ${ }^{100}$ A. C. Schaffer, ${ }^{65}$ D. Schaile, ${ }^{114}$ R. D. Schamberger,${ }^{155}$ N. Scharmberg, ${ }^{101}$ V. A. Schegelsky, ${ }^{138}$ D. Scheirich, ${ }^{143}$ F. Schenck, ${ }^{19}$ M. Schernau, ${ }^{171}$ C. Schiavi, ${ }^{55 b, 55 a}$ S. Schier, ${ }^{146}$ L. K. Schildgen, ${ }^{24}$ Z. M. Schillaci, ${ }^{26}$ E. J. Schioppa,${ }^{36}$ M. Schioppa,${ }^{41 b, 41 a}$ K. E. Schleicher, ${ }^{52}$ S. Schlenker, ${ }^{36}$ K. R. Schmidt-Sommerfeld, ${ }^{115}$ K. Schmieden, ${ }^{36}$ C. Schmitt, ${ }^{100}$ S. Schmitt,${ }^{46}$ S. Schmitz, ${ }^{100}$ J. C. Schmoeckel, ${ }^{46}$ U. Schnoor, ${ }^{52}$ L. Schoeffel, ${ }^{145}$ A. Schoening, ${ }^{61 b}$ P. G. Scholer, ${ }^{52}$ E. Schopf, ${ }^{135}$ M. Schott, ${ }^{100}$ J. F. P. Schouwenberg, ${ }^{119}$ J. Schovancova, ${ }^{36}$ S. Schramm, ${ }^{54}$ F. Schroeder, ${ }^{182}$ A. Schulte, ${ }^{100}$ H-C. Schultz-Coulon, ${ }^{61 a}$ M. Schumacher, ${ }^{52}$ 
B. A. Schumm, ${ }^{146}$ Ph. Schune, ${ }^{145}$ A. Schwartzman, ${ }^{153}$ T. A. Schwarz, ${ }^{106}$ Ph. Schwemling, ${ }^{145}$ R. Schwienhorst, ${ }^{107}$ A. Sciandra, ${ }^{146}$ G. Sciolla, ${ }^{26}$ M. Scodeggio, ${ }^{46}$ M. Scornajenghi, ${ }^{41 b, 41 a}$ F. Scuri, ${ }^{72 a}$ F. Scutti, ${ }^{105}$ L. M. Scyboz, ${ }^{115}$ C. D. Sebastiani, ${ }^{73 a, 73 b}$ P. Seema, ${ }^{19}$ S. C. Seidel, ${ }^{118}$ A. Seiden, ${ }^{146}$ B. D. Seidlitz, ${ }^{29}$ T. Seiss, ${ }^{37}$ J. M. Seixas, ${ }^{81 b}$ G. Sekhniaidze, ${ }^{70 a}$ K. Sekhon, ${ }^{106}$ S. J. Sekula, ${ }^{42}$ N. Semprini-Cesari, ${ }^{23 b, 23 a}$ S. Sen, ${ }^{49}$ S. Senkin, ${ }^{38}$ C. Serfon, ${ }^{77}$ L. Serin, ${ }^{65}$ L. Serkin, ${ }^{67 a, 67 b}$ M. Sessa, ${ }^{60 a}$ H. Severini, ${ }^{129}$ T. Šfiligoj, ${ }^{92}$ F. Sforza, ${ }^{55 b, 55 a}$ A. Sfyrla, ${ }^{54}$ E. Shabalina, ${ }^{53}$ J. D. Shahinian, ${ }^{146}$ N. W. Shaikh, ${ }^{45,45 b}$ D. Shaked Renous, ${ }^{180}$ L. Y. Shan, ${ }^{15 a}$ R. Shang, ${ }^{173}$ J. T. Shank, ${ }^{25}$ M. Shapiro, ${ }^{18}$ A. Sharma, ${ }^{135}$ A. S. Sharma, ${ }^{1}$ P. B. Shatalov, ${ }^{124}$ K. Shaw, ${ }^{156}$ S. M. Shaw, ${ }^{101}$ A. Shcherbakova, ${ }^{138}$ M. Shehade, ${ }^{180}$ Y. Shen, ${ }^{129}$ N. Sherafati, ${ }^{34}$ A. D. Sherman, ${ }^{25}$ P. Sherwood, ${ }^{95}$ L. Shi, ${ }^{158, n n}$ S. Shimizu, ${ }^{82}$ C. O. Shimmin, ${ }^{183}$ Y. Shimogama, ${ }^{179}$ M. Shimojima, ${ }^{116}$ I. P. J. Shipsey, ${ }^{135}$ S. Shirabe, ${ }^{88}$ M. Shiyakova, ${ }^{80,00}$ J. Shlomi, ${ }^{180}$ A. Shmeleva, ${ }^{111}$ M. J. Shochet, ${ }^{37}$ J. Shojaii, ${ }^{105}$ D. R. Shope, ${ }^{129}$ S. Shrestha, ${ }^{127}$ E. M. Shrif, ${ }^{33 e}$ E. Shulga, ${ }^{180}$ P. Sicho, ${ }^{141}$ A. M. Sickles, ${ }^{173}$ P. E. Sidebo, ${ }^{154}$

E. Sideras Haddad, ${ }^{33 e}$ O. Sidiropoulou, ${ }^{36}$ A. Sidoti, ${ }^{23 b, 23 a}$ F. Siegert, ${ }^{48}$ Dj. Sijacki, ${ }^{16}$ M. Jr. Silva, ${ }^{181}$ M. V. Silva Oliveira, ${ }^{81 a}$ S. B. Silverstein, ${ }^{45 a}$ S. Simion, ${ }^{65}$ E. Simioni, ${ }^{100}$ R. Simoniello, ${ }^{100}$ S. Simsek, ${ }^{12 b}$ P. Sinervo, ${ }^{167}$ V. Sinetckii, ${ }^{113,111}$ N. B. Sinev, ${ }^{132}$ M. Sioli, ${ }^{23 b, 23 a}$ I. Siral, ${ }^{106}$ S. Yu. Sivoklokov, ${ }^{113}$ J. Sjölin, ${ }^{45 a, 45 b}$ E. Skorda, ${ }^{97}$ P. Skubic, ${ }^{129}$ M. Slawinska, ${ }^{85}$ K. Sliwa, ${ }^{170}$ R. Slovak, ${ }^{143}$ V. Smakhtin, ${ }^{180}$ B. H. Smart, ${ }^{144}$ J. Smiesko, ${ }^{28 a}$ N. Smirnov, ${ }^{112}$ S. Yu. Smirnov, ${ }^{112}$ Y. Smirnov, ${ }^{112}$ L. N. Smirnova, ${ }^{113, p p}$ O. Smirnova, ${ }^{97}$ J. W. Smith, ${ }^{53}$ M. Smizanska, ${ }^{90}$ K. Smolek, ${ }^{142}$ A. Smykiewicz, ${ }^{85}$ A. A. Snesarev, ${ }^{111}$ H. L. Snoek, ${ }^{120}$ I. M. Snyder, ${ }^{132}$ S. Snyder, ${ }^{29}$ R. Sobie, ${ }^{176, p}$ A. Soffer, ${ }^{161}$ A. Søgaard, ${ }^{50}$ F. Sohns, ${ }^{53}$ C. A. Solans Sanchez, ${ }^{36}$ E. Yu. Soldatov, ${ }^{112}$ U. Soldevila, ${ }^{174}$ A. A. Solodkov, ${ }^{123}$ A. Soloshenko, ${ }^{80}$ O. V. Solovyanov, ${ }^{123}$ V. Solovyev, ${ }^{138}$ P. Sommer, ${ }^{149}$ H. Son, ${ }^{170}$ W. Song, ${ }^{144}$ W. Y. Song, ${ }^{168 b}$ A. Sopczak, ${ }^{142}$ F. Sopkova, ${ }^{28 b}$ C. L. Sotiropoulou, ${ }^{72 a, 72 b}$ S. Sottocornola, ${ }^{71 a, 71 b}$ R. Soualah, ${ }^{67 \mathrm{a}, 67 \mathrm{c}, \mathrm{qq}}$ A. M. Soukharev, ${ }^{122 \mathrm{~b}, 122 \mathrm{a}}$ D. South, ${ }^{46}$ S. Spagnolo, ${ }^{68 \mathrm{a}, 68 \mathrm{~b}}$ M. Spalla, ${ }^{115}$ M. Spangenberg, ${ }^{178}$ F. Spanò, ${ }^{94}$ D. Sperlich, ${ }^{52}$ T. M. Spieker, ${ }^{61 a}$ R. Spighi, ${ }^{23 b}$ G. Spigo, ${ }^{36}$ M. Spina, ${ }^{156}$ D. P. Spiteri, ${ }^{57}$ M. Spousta, ${ }^{143}$ A. Stabile, ${ }^{69 a, 69 b}$ B. L. Stamas, ${ }^{121}$ R. Stamen, ${ }^{61 a}$ M. Stamenkovic, ${ }^{120}$ E. Stanecka, ${ }^{85}$ B. Stanislaus, ${ }^{135}$ M. M. Stanitzki, ${ }^{46}$ M. Stankaityte, ${ }^{135}$ B. Stapf, ${ }^{120}$ E. A. Starchenko, ${ }^{123}$ G. H. Stark, ${ }^{146}$ J. Stark, ${ }^{58}$ S. H. Stark, ${ }^{40}$ P. Staroba, ${ }^{141}$ P. Starovoitov, ${ }^{61 a}$ S. Stärz, ${ }^{104}$ R. Staszewski, ${ }^{85}$ G. Stavropoulos, ${ }^{44}$ M. Stegler, ${ }^{46}$ P. Steinberg, ${ }^{29}$ A. L. Steinhebel, ${ }^{132}$ B. Stelzer, ${ }^{152}$ H. J. Stelzer, ${ }^{139}$ O. Stelzer-Chilton, ${ }^{168 a}$ H. Stenzel, ${ }^{56}$ T. J. Stevenson, ${ }^{156}$ G. A. Stewart, ${ }^{36}$ M. C. Stockton, ${ }^{36}$ G. Stoicea, ${ }^{27 b}$ M. Stolarski, ${ }^{140 a}$ S. Stonjek, ${ }^{115}$ A. Straessner, ${ }^{48}$ J. Strandberg, ${ }^{154}$ S. Strandberg, ${ }^{45,45 b}$ M. Strauss, ${ }^{129}$ P. Strizenec, ${ }^{28 b}$ R. Ströhmer, ${ }^{177}$ D. M. Strom, ${ }^{132}$ R. Stroynowski, ${ }^{42}$ A. Strubig, ${ }^{50}$ S. A. Stucci, ${ }^{29}$ B. Stugu, ${ }^{17}$ J. Stupak, ${ }^{129}$ N. A. Styles, ${ }^{46}$ D. Su, ${ }^{153}$ S. Suchek, ${ }^{61 \mathrm{a}}$ V. V. Sulin, ${ }^{11}$ M. J. Sullivan, ${ }^{91}$ D. M. S. Sultan, ${ }^{54}$ S. Sultansoy, ${ }^{4 \mathrm{c}}$ T. Sumida, ${ }^{86}$ S. Sun, ${ }^{106}$ X. Sun, ${ }^{3}$ K. Suruliz, ${ }^{156}$ C. J. E. Suster, ${ }^{157}$ M. R. Sutton, ${ }^{156}$ S. Suzuki, ${ }^{82}$ M. Svatos, ${ }^{141}$ M. Swiatlowski, ${ }^{37}$ S. P. Swift, ${ }^{2}$ T. Swirski, ${ }^{177}$ A. Sydorenko, ${ }^{100}$ I. Sykora, ${ }^{28 a}$ M. Sykora, ${ }^{143}$ T. Sykora, ${ }^{143}$ D. Ta, ${ }^{100}$ K. Tackmann, ${ }^{46, r r}$ J. Taenzer, ${ }^{161}$ A. Taffard, ${ }^{171}$ R. Tafirout, ${ }^{168 a}$ H. Takai, ${ }^{29}$ R. Takashima, ${ }^{87}$ K. Takeda, ${ }^{83}$ T. Takeshita, ${ }^{150}$ E. P. Takeva, ${ }^{50}$ Y. Takubo, ${ }^{82}$ M. Talby, ${ }^{102}$ A. A. Talyshev, ${ }^{122 b, 122 a}$ N. M. Tamir, ${ }^{161}$ J. Tanaka, ${ }^{163}$ M. Tanaka, ${ }^{165}$ R. Tanaka, ${ }^{65}$ S. Tapia Araya, ${ }^{173}$ S. Tapprogge, ${ }^{100}$ A. Tarek Abouelfadl Mohamed, ${ }^{136}$ S. Tarem, ${ }^{160}$ K. Tariq, ${ }^{60 b}$ G. Tarna, ${ }^{27 b, s s}$ G. F. Tartarelli, ${ }^{69 a}$ P. Tas, ${ }^{143}$ M. Tasevsky, ${ }^{141}$ T. Tashiro, ${ }^{86}$ E. Tassi, ${ }^{41 \mathrm{~b}, 41 \mathrm{a}}$ A. Tavares Delgado, ${ }^{140 \mathrm{a}, 140 \mathrm{~b}}$ Y. Tayalati, ${ }^{35 \mathrm{e}}$ A. J. Taylor, ${ }^{50}$ G. N. Taylor, ${ }^{105}$ W. Taylor, ${ }^{168 \mathrm{~b}}$ A. S. Tee, ${ }^{90}$ R. Teixeira De Lima, ${ }^{153}$ P. Teixeira-Dias, ${ }^{94}$ H. Ten Kate, ${ }^{36}$ J. J. Teoh, ${ }^{120}$ S. Terada, ${ }^{82}$ K. Terashi, ${ }^{163}$ J. Terron, ${ }^{99}$ S. Terzo, ${ }^{14}$ M. Testa, ${ }^{51}$ R. J. Teuscher, ${ }^{167, p}$ S. J. Thais, ${ }^{183}$ T. Theveneaux-Pelzer, ${ }^{46}$ F. Thiele, ${ }^{40}$ D. W. Thomas, ${ }^{94}$ J. O. Thomas, ${ }^{42}$ J. P. Thomas, ${ }^{21}$ A. S. Thompson, ${ }^{57}$ P. D. Thompson, ${ }^{21}$ L. A. Thomsen, ${ }^{183}$ E. Thomson, ${ }^{137}$ E. J. Thorpe, ${ }^{93}$ R. E. Ticse Torres, ${ }^{53}$ V. O. Tikhomirov, ${ }^{111, \text { tt }}$ Yu. A. Tikhonov, ${ }^{122 b, 122 \mathrm{a}}$ S. Timoshenko, ${ }^{112}$ P. Tipton, ${ }^{183}$ S. Tisserant, ${ }^{102}$ K. Todome, ${ }^{23 b, 23 a}$ S. Todorova-Nova, ${ }^{5}$ S. Todt, ${ }^{48}$ J. Tojo, ${ }^{88}$ S. Tokár, ${ }^{28 a}$ K. Tokushuku, ${ }^{82}$ E. Tolley, ${ }^{127}$ K. G. Tomiwa, ${ }^{33 e}$ M. Tomoto, ${ }^{117}$ L. Tompkins, ${ }^{153, f f}$ B. Tong, ${ }^{59}$ P. Tornambe, ${ }^{103}$ E. Torrence, ${ }^{132}$ H. Torres, ${ }^{48}$ E. Torró Pastor, ${ }^{148}$ C. Tosciri, ${ }^{135}$ J. Toth, ${ }^{102, \text { uu }}$ D. R. Tovey, ${ }^{149}$ A. Traeet, ${ }^{17}$ C. J. Treado, ${ }^{125}$ T. Trefzger, ${ }^{177}$ F. Tresoldi, ${ }^{156}$ A. Tricoli, ${ }^{29}$ I. M. Trigger, ${ }^{168 a}$ S. Trincaz-Duvoid, ${ }^{136}$ W. Trischuk, ${ }^{167}$ B. Trocmé, ${ }^{58}$ A. Trofymov, ${ }^{145}$ C. Troncon, ${ }^{69 a}$ M. Trovatelli, ${ }^{176}$ F. Trovato, ${ }^{156}$ L. Truong, ${ }^{33 \mathrm{c}}$ M. Trzebinski, ${ }^{85}$ A. Trzupek, ${ }^{85}$ F. Tsai, ${ }^{46}$ J. C-L. Tseng, ${ }^{135}$ P. V. Tsiareshka, ${ }^{108, \text { hh }}$ A. Tsirigotis, ${ }^{162}$ V. Tsiskaridze, ${ }^{155}$ E. G. Tskhadadze, ${ }^{159 a}$ M. Tsopoulou, ${ }^{162}$ I. I. Tsukerman, ${ }^{124}$ V. Tsulaia, ${ }^{18}$ S. Tsuno, ${ }^{82}$ D. Tsybychev, ${ }^{155}$ Y. Tu, ${ }^{63 \mathrm{~b}}$ A. Tudorache, ${ }^{27 \mathrm{~b}}$ V. Tudorache, ${ }^{27 \mathrm{~b}}$ T. T. Tulbure, ${ }^{27 \mathrm{a}}$ A. N. Tuna, ${ }^{59}$ S. Turchikhin, ${ }^{80}$ D. Turgeman, ${ }^{180}$ I. Turk Cakir, ${ }^{4 b, v v}$ R. J. Turner, ${ }^{21}$ R. Turra, ${ }^{69 a}$ P. M. Tuts, ${ }^{39}$ S. Tzamarias, ${ }^{162}$ E. Tzovara, ${ }^{100}$ G. Ucchielli, ${ }^{47}$ K. Uchida, ${ }^{163}$ I. Ueda, ${ }^{82}$ M. Ughetto, ${ }^{45 a, 45 b}$ F. Ukegawa, ${ }^{169}$ G. Unal, ${ }^{36}$ A. Undrus, ${ }^{29}$ G. Unel, ${ }^{171}$ F. C. Ungaro, ${ }^{105}$ Y. Unno, ${ }^{82}$ K. Uno, ${ }^{163}$ J. Urban, ${ }^{28 b}$ P. Urquijo, ${ }^{105}$ G. Usai, ${ }^{8}$ Z. Uysal, ${ }^{12 \mathrm{~d}}$ L. Vacavant, ${ }^{102}$ V. Vacek, ${ }^{142}$ B. Vachon, ${ }^{104}$ K. O. H. Vadla, ${ }^{134}$ A. Vaidya, ${ }^{95}$ C. Valderanis, ${ }^{114}$ E. Valdes Santurio, ${ }^{45,45 b}$ M. Valente, ${ }^{54}$ S. Valentinetti, ${ }^{23 b, 23 a}$ A. Valero, ${ }^{174}$ L. Valéry, ${ }^{46}$ R. A. Vallance, ${ }^{21}$ A. Vallier, ${ }^{36}$ J. A. Valls Ferrer, ${ }^{174}$ T. R. Van Daalen, ${ }^{14}$ P. Van Gemmeren, ${ }^{6}$ I. Van Vulpen, ${ }^{120}$ M. Vanadia, ${ }^{74 a, 74 b}$ W. Vandelli, ${ }^{36}$ 
E. R. Vandewall, ${ }^{130}$ A. Vaniachine, ${ }^{166}$ D. Vannicola,${ }^{73 a, 73 b}$ R. Vari, ${ }^{73 a}$ E. W. Varnes, ${ }^{7}$ C. Varni,${ }^{55 b, 55 a}$ T. Varol, ${ }^{158}$ D. Varouchas, ${ }^{65}$ K. E. Varvell, ${ }^{157}$ M. E. Vasile, ${ }^{27 b}$ G. A. Vasquez, ${ }^{176}$ J. G. Vasquez, ${ }^{183}$ F. Vazeille, ${ }^{38}$ D. Vazquez Furelos, ${ }^{14}$ T. Vazquez Schroeder, ${ }^{36}$ J. Veatch ${ }^{53}$ V. Vecchio, ${ }^{75 a, 75 b}$ M. J. Veen, ${ }^{120}$ L. M. Veloce, ${ }^{167}$ F. Veloso, ${ }^{140 a, 140 c}$ S. Veneziano, ${ }^{73 a}$ A. Ventura, ${ }^{68 a, 68 b}$ N. Venturi, ${ }^{36}$ A. Verbytskyi, ${ }^{115}$ V. Vercesi, ${ }^{71 a}$ M. Verducci, ${ }^{72 a, 72 b}$ C. M. Vergel Infante, ${ }^{79}$ C. Vergis, ${ }^{24}$ W. Verkerke, ${ }^{120}$ A. T. Vermeulen, ${ }^{120}$ J. C. Vermeulen, ${ }^{120}$ M. C. Vetterli, ${ }^{152, d}$ N. Viaux Maira ${ }^{147 \mathrm{~d}}$ M. Vicente Barreto Pinto ${ }^{54}$ T. Vickey, ${ }^{149}$ O. E. Vickey Boeriu, ${ }^{149}$ G. H. A. Viehhauser, ${ }^{135}$ L. Vigani, ${ }^{61 b}$ M. Villa, ${ }^{23 b, 23 a}$ M. Villaplana Perez, ${ }^{69 a, 69 b}$ E. Vilucchi, ${ }^{51}$ M. G. Vincter, ${ }^{34}$ G. S. Virdee, ${ }^{21}$ A. Vishwakarma, ${ }^{46}$ C. Vittori, ${ }^{23 b, 23 a}$ I. Vivarelli, ${ }^{156}$ M. Vogel, ${ }^{182}$ P. Vokac, ${ }^{142}$ S. E. von Buddenbrock, ${ }^{33 \mathrm{e}}$ E. Von Toerne, ${ }^{24}$ V. Vorobel, ${ }^{143}$ K. Vorobev, ${ }^{112}$ M. Vos, ${ }^{174}$ J. H. Vossebeld,${ }^{91}$ M. Vozak, ${ }^{101}$

N. Vranjes, ${ }^{16}$ M. Vranjes Milosavljevic, ${ }^{16}$ V. Vrba, ${ }^{142}$ M. Vreeswijk, ${ }^{120}$ R. Vuillermet, ${ }^{36}$ I. Vukotic, ${ }^{37}$ P. Wagner ${ }^{24}$

W. Wagner, ${ }^{182}$ J. Wagner-Kuhr, ${ }^{114}$ S. Wahdan, ${ }^{182}$ H. Wahlberg, ${ }^{89}$ V. M. Walbrecht, ${ }^{115}$ J. Walder, ${ }^{90}$ R. Walker, ${ }^{114}$

S. D. Walker, ${ }^{94}$ W. Walkowiak, ${ }^{151}$ V. Wallangen, ${ }^{45 a, 45 b}$ A. M. Wang,${ }^{59}$ C. Wang, ${ }^{60 c}$ C. Wang, ${ }^{60 b}$ F. Wang, ${ }^{181}$ H. Wang, ${ }^{18}$ H. Wang, ${ }^{3}$ J. Wang, ${ }^{63 a}$ J. Wang, ${ }^{157}$ J. Wang, ${ }^{61 b}$ P. Wang, ${ }^{42}$ Q. Wang, ${ }^{129}$ R.-J. Wang, ${ }^{100}$ R. Wang, ${ }^{60 a}$ R. Wang, ${ }^{6}$ S. M. Wang, ${ }^{158}$ W. T. Wang, ${ }^{60 \mathrm{a}}$ W. Wang, ${ }^{15 \mathrm{c}, \mathrm{ww}}$ W. X. Wang, ${ }^{60 \mathrm{a}, \mathrm{ww}}$ Y. Wang, ${ }^{60, \mathrm{axx}}$ Z. Wang, ${ }^{60 \mathrm{c}}$ C. Wanotayaroj ${ }^{46}$ A. Warburton, ${ }^{104}$

C. P. Ward, ${ }^{32}$ D. R. Wardrope, ${ }^{95}$ N. Warrack, ${ }^{57}$ A. Washbrook, ${ }^{50}$ A. T. Watson, ${ }^{21}$ M. F. Watson, ${ }^{21}$ G. Watts, ${ }^{148}$

B. M. Waugh,${ }^{95}$ A. F. Webb, ${ }^{11}$ S. Webb, ${ }^{100}$ C. Weber, ${ }^{183}$ M. S. Weber, ${ }^{20}$ S. A. Weber, ${ }^{34}$ S. M. Weber, ${ }^{61 a}$ A. R. Weidberg, ${ }^{135}$ J. Weingarten,${ }^{47} \mathrm{M}$. Weirich, ${ }^{100} \mathrm{C}$. Weiser, ${ }^{52}$ P. S. Wells, ${ }^{36}$ T. Wenaus, ${ }^{29}$ T. Wengler, ${ }^{36}$ S. Wenig, ${ }^{36} \mathrm{~N}$. Wermes, ${ }^{24}$ M. D. Werner, ${ }^{79}$ M. Wessels, ${ }^{61 a}$ T. D. Weston, ${ }^{20}$ K. Whalen, ${ }^{132}$ N. L. Whallon, ${ }^{148}$ A. M. Wharton, ${ }^{90}$ A. S. White, ${ }^{106}$ A. White,${ }^{8}$ M. J. White, ${ }^{1}$ D. Whiteson, ${ }^{171}$ B. W. Whitmore,${ }^{90}$ W. Wiedenmann, ${ }^{181}$ M. Wielers,${ }^{144}$ N. Wieseotte, ${ }^{100}$ C. Wiglesworth ${ }^{40}$ L. A. M. Wiik-Fuchs,${ }^{52}$ F. Wilk, ${ }^{101}$ H. G. Wilkens, ${ }^{36}$ L. J. Wilkins, ${ }^{94}$ H. H. Williams, ${ }^{137}$ S. Williams, ${ }^{32}$ C. Willis, ${ }^{107}$ S. Willocq, ${ }^{103}$ J. A. Wilson, ${ }^{21}$ I. Wingerter-Seez, ${ }^{5}$ E. Winkels, ${ }^{156}$ F. Winklmeier, ${ }^{132}$ O. J. Winston, ${ }^{156}$

B. T. Winter, ${ }^{52}$ M. Wittgen, ${ }^{153}$ M. Wobisch, ${ }^{96}$ A. Wolf, ${ }^{100}$ T. M. H. Wolf, ${ }^{120}$ R. Wolff, ${ }^{102}$ R. Wölker, ${ }^{135}$ J. Wollrath, ${ }^{52}$ M. W. Wolter ${ }^{85}$ H. Wolters, ${ }^{140 a, 140 c}$ V. W. S. Wong, ${ }^{175}$ N. L. Woods, ${ }^{146}$ S. D. Worm, ${ }^{21}$ B. K. Wosiek, ${ }^{85}$ K. W. Woźniak, ${ }^{85}$ K. Wraight,${ }^{57}$ S. L. Wu, ${ }^{181}$ X. Wu, ${ }^{54}$ Y. Wu, ${ }^{60 a}$ T. R. Wyatt,,${ }^{101}$ B. M. Wynne, ${ }^{50}$ S. Xella, ${ }^{40}$ Z. Xi, ${ }^{106}$ L. Xia, ${ }^{178}$ X. Xiao, ${ }^{106}$ I. Xiotidis, ${ }^{156}$ D. Xu, ${ }^{15 a}$ H. Xu, ${ }^{60 a, s s}$ L. Xu, ${ }^{29}$ T. Xu, ${ }^{145}$ W. Xu, ${ }^{106}$ Z. Xu, ${ }^{60 b}$ Z. Xu, ${ }^{153}$ B. Yabsley, ${ }^{157}$ S. Yacoob, ${ }^{33 a}$

K. Yajima, ${ }^{133}$ D. P. Yallup, ${ }^{95}$ D. Yamaguchi, ${ }^{165}$ Y. Yamaguchi, ${ }^{165}$ A. Yamamoto, ${ }^{82}$ M. Yamatani, ${ }^{163}$ T. Yamazaki, ${ }^{163}$ Y. Yamazaki, ${ }^{83}$ Z. Yan, ${ }^{25}$ H. J. Yang, ${ }^{60 c, 60 d}$ H. T. Yang, ${ }^{18}$ S. Yang, ${ }^{78}$ X. Yang, ${ }^{60 b, 58}$ Y. Yang, ${ }^{163}$ W-M. Yao, ${ }^{18}$ Y. C. Yap ${ }^{46}$ Y. Yasu, ${ }^{82}$ E. Yatsenko, ${ }^{60 c, 60 d}$ J. Ye, ${ }^{42}$ S. Ye ${ }^{29}$ I. Yeletskikh ${ }^{80}$ M. R. Yexley, ${ }^{90}$ E. Yigitbasi, ${ }^{25} \mathrm{~K}$. Yorita, ${ }^{179} \mathrm{~K}$. Yoshihara, ${ }^{137}$ C. J. S. Young, ${ }^{36}$ C. Young, ${ }^{153}$ J. Yu ${ }^{79}$ R. Yuan,${ }^{60 b, y y}$ X. Yue ${ }^{61 a}$ S. P. Y. Yuen, ${ }^{24}$ M. Zaazoua, ${ }^{35 e}$ B. Zabinski, ${ }^{85}$ G. Zacharis,${ }^{10}$ E. Zaffaroni, ${ }^{54}$ J. Zahreddine, ${ }^{136}$ A. M. Zaitsev, ${ }^{123, \mathrm{k}}$ T. Zakareishvili, ${ }^{159 b}$ N. Zakharchuk, ${ }^{34}$ S. Zambito, ${ }^{59}$ D. Zanzi, ${ }^{36}$ D. R. Zaripovas, ${ }^{57}$ S. V. Zeißner, ${ }^{47}$ C. Zeitnitz, ${ }^{182}$ G. Zemaityte, ${ }^{135}$ J. C. Zeng, ${ }^{173}$ O. Zenin, ${ }^{123}$ T. Ženiš, ${ }^{28 a}$ D. Zerwas, ${ }^{65}$ M. Zgubič ${ }^{135}$ B. Zhang, ${ }^{15 \mathrm{c}}$ D. F. Zhang, ${ }^{15 \mathrm{~b}}$ G. Zhang, ${ }^{15 \mathrm{~b}}$ H. Zhang, ${ }^{15 \mathrm{c}}$ J. Zhang, ${ }^{6}$ L. Zhang, ${ }^{15 \mathrm{c}}$ L. Zhang, ${ }^{60 \mathrm{a}}$ M. Zhang, ${ }^{173}$ R. Zhang, ${ }^{24}$ X. Zhang, ${ }^{60 \mathrm{~b}}$ Y. Zhang, ${ }^{15 a, 15 \mathrm{~d}}$ Z. Zhang, ${ }^{63 \mathrm{a}}$ Z. Zhang, ${ }^{65}$ P. Zhao, ${ }^{49}$ Y. Zhao, ${ }^{60 \mathrm{~b}}$ Z. Zhao, ${ }^{60 \mathrm{a}}$ A. Zhemchugov ${ }^{80}$ Z. Zheng, ${ }^{106}$ D. Zhong, ${ }^{173}$ B. Zhou, ${ }^{106}$ C. Zhou ${ }^{181}$ M. S. Zhou, ${ }^{15 a, 15 d}$ M. Zhou, ${ }^{155}$ N. Zhou, ${ }^{60 c}$ Y. Zhou, ${ }^{7}$ C. G. Zhu, ${ }^{60 b}$ C. Zhu, ${ }^{15,15 \mathrm{~d}}$ H. L. Zhu, ${ }^{60 \mathrm{a}}$ H. Zhu, ${ }^{15 \mathrm{a}} \mathrm{J}$. Zhu ${ }^{106}{ }^{1}$ Y. Zhu, ${ }^{60 \mathrm{a}}$ X. Zhuang, ${ }^{15 \mathrm{a}} \mathrm{K}$. Zhukov, ${ }^{111}$ V. Zhulanov, ${ }^{122 b, 122 \mathrm{a}}$ D. Zieminska, ${ }^{66}$ N. I. Zimine, ${ }^{80}$ S. Zimmermann, ${ }^{52}$ Z. Zinonos, ${ }^{115}$ M. Ziolkowski, ${ }^{151}$ L. Živković, ${ }^{16}$ G. Zobernig, ${ }^{181}$ A. Zoccoli, ${ }^{23 b, 23 a}$ K. Zoch, ${ }^{53}$ T. G. Zorbas, ${ }^{149}$ R. Zou, ${ }^{37}$ and L. Zwalinski ${ }^{36}$

(ATLAS Collaboration)

\footnotetext{
${ }^{1}$ Department of Physics, University of Adelaide, Adelaide, Australia ${ }^{2}$ Physics Department, SUNY Albany, Albany NY, United States of America

${ }^{3}$ Department of Physics, University of Alberta, Edmonton AB, Canada

${ }^{4 a}$ Department of Physics, Ankara University, Ankara, Turkey

${ }^{4 b}$ Istanbul Aydin University, Application and Research Center for Advanced Studies, Istanbul, Turkey

${ }^{4 \mathrm{c}}$ Division of Physics, TOBB University of Economics and Technology, Ankara, Turkey

${ }^{5}$ LAPP, Université Grenoble Alpes, Université Savoie Mont Blanc, CNRS/IN2P3, Annecy, France

${ }^{6}$ High Energy Physics Division, Argonne National Laboratory, Argonne IL, United States of America

${ }^{7}$ Department of Physics, University of Arizona, Tucson AZ, United States of America

${ }^{8}$ Department of Physics, University of Texas at Arlington, Arlington TX, United States of America

${ }^{9}$ Physics Department, National and Kapodistrian University of Athens, Athens, Greece

${ }^{10}$ Physics Department, National Technical University of Athens, Zografou, Greece
} 
${ }^{11}$ Department of Physics, University of Texas at Austin, Austin TX, United States of America

${ }^{12 a}$ Bahcesehir University, Faculty of Engineering and Natural Sciences, Istanbul, Turkey

${ }^{12 \mathrm{~b}}$ Istanbul Bilgi University, Faculty of Engineering and Natural Sciences, Istanbul, Turkey

${ }^{12 \mathrm{c}}$ Department of Physics, Bogazici University, Istanbul, Turkey

${ }^{12 \mathrm{~d}}$ Department of Physics Engineering, Gaziantep University, Gaziantep, Turkey

${ }^{13}$ Institute of Physics, Azerbaijan Academy of Sciences, Baku, Azerbaijan

${ }^{14}$ Institut de Física d'Altes Energies (IFAE),

Barcelona Institute of Science and Technology, Barcelona, Spain

${ }^{15 a}$ Institute of High Energy Physics, Chinese Academy of Sciences, Beijing, China

${ }^{15 \mathrm{~b}}$ Physics Department, Tsinghua University, Beijing, China

${ }^{15 \mathrm{c}}$ Department of Physics, Nanjing University, Nanjing, China

${ }^{15 \mathrm{~d}}$ University of Chinese Academy of Science (UCAS), Beijing, China

${ }^{16}$ Institute of Physics, University of Belgrade, Belgrade, Serbia

${ }^{17}$ Department for Physics and Technology, University of Bergen, Bergen, Norway

${ }^{18}$ Physics Division, Lawrence Berkeley National Laboratory and University of California, Berkeley CA, United States of America

${ }^{19}$ Institut für Physik, Humboldt Universität zu Berlin, Berlin, Germany

${ }^{20}$ Albert Einstein Center for Fundamental Physics and Laboratory for High Energy Physics, University of Bern, Bern, Switzerland

${ }^{21}$ School of Physics and Astronomy, University of Birmingham, Birmingham, United Kingdom

${ }^{22 \mathrm{a}}$ Facultad de Ciencias y Centro de Investigaciónes, Universidad Antonio Nariño, Bogotá, Colombia

${ }^{22 \mathrm{~b}}$ Departamento de Física, Universidad Nacional de Colombia, Bogotá, Colombia, Colombia

${ }^{23 a}$ INFN Bologna and Universita' di Bologna, Dipartimento di Fisica, Italy

${ }^{23 \mathrm{~b}}$ INFN Sezione di Bologna, Italy

${ }^{24}$ Physikalisches Institut, Universität Bonn, Bonn, Germany

${ }^{25}$ Department of Physics, Boston University, Boston MA, United States of America

${ }^{26}$ Department of Physics, Brandeis University, Waltham MA, United States of America

${ }^{27}$ Transilvania University of Brasov, Brasov, Romania

${ }^{27 \mathrm{~b}}$ Horia Hulubei National Institute of Physics and Nuclear Engineering, Bucharest, Romania

${ }^{27 \mathrm{c}}$ Department of Physics, Alexandru Ioan Cuza University of Iasi, Iasi, Romania

${ }^{27 \mathrm{~d}}$ National Institute for Research and Development of Isotopic and Molecular Technologies, Physics Department, Cluj-Napoca, Romania

${ }^{27 \mathrm{e}}$ University Politehnica Bucharest, Bucharest, Romania

${ }^{27 \mathrm{f}}$ West University in Timisoara, Timisoara, Romania

${ }^{28 a}$ Faculty of Mathematics, Physics and Informatics, Comenius University, Bratislava, Slovak Republic

${ }^{28 \mathrm{~b}}$ Department of Subnuclear Physics, Institute of Experimental Physics of the Slovak Academy of Sciences, Kosice, Slovak Republic

${ }^{29}$ Physics Department, Brookhaven National Laboratory, Upton NY, United States of America

${ }^{30}$ Departamento de Física, Universidad de Buenos Aires, Buenos Aires, Argentina

${ }^{31}$ California State University, CA, United States of America

${ }^{32}$ Cavendish Laboratory, University of Cambridge, Cambridge, United Kingdom

${ }^{33 a}$ Department of Physics, University of Cape Town, Cape Town, South Africa

${ }^{33 \mathrm{~b}}$ iThemba Labs, Western Cape, South Africa

${ }^{33 c}$ Department of Mechanical Engineering Science, University of Johannesburg, Johannesburg, South Africa

${ }^{33 \mathrm{~d}}$ University of South Africa, Department of Physics, Pretoria, South Africa

${ }^{33 \mathrm{e}}$ School of Physics, University of the Witwatersrand, Johannesburg, South Africa

${ }^{34}$ Department of Physics, Carleton University, Ottawa ON, Canada

${ }^{35 a}$ Faculté des Sciences Ain Chock, Réseau Universitaire de Physique des Hautes Energies-Université Hassan II, Casablanca, Morocco

${ }^{35 \mathrm{~b}}$ Faculté des Sciences, Université Ibn-Tofail, Kénitra, Morocco

${ }^{35 c}$ Faculté des Sciences Semlalia, Université Cadi Ayyad, LPHEA-Marrakech, Morocco

${ }^{35 \mathrm{~d}}$ Faculté des Sciences, Université Mohamed Premier and LPTPM, Oujda, Morocco

${ }^{35 \mathrm{e}}$ Faculté des sciences, Université Mohammed V, Rabat, Morocco

${ }^{36}$ CERN, Geneva, Switzerland

${ }^{37}$ Enrico Fermi Institute, University of Chicago, Chicago IL, United States of America

${ }^{38}$ LPC, Université Clermont Auvergne, CNRS/IN2P3, Clermont-Ferrand, France

${ }^{39}$ Nevis Laboratory, Columbia University, Irvington NY, United States of America

${ }^{40}$ Niels Bohr Institute, University of Copenhagen, Copenhagen, Denmark

${ }^{41 a}$ Dipartimento di Fisica, Università della Calabria, Rende, Italy 
${ }^{41 \mathrm{~b}}$ INFN Gruppo Collegato di Cosenza, Laboratori Nazionali di Frascati, Italy

${ }^{42}$ Physics Department, Southern Methodist University, Dallas TX, United States of America

${ }^{43}$ Physics Department, University of Texas at Dallas, Richardson TX, United States of America

${ }^{44}$ National Centre for Scientific Research "Demokritos”, Agia Paraskevi, Greece

${ }^{45 a}$ Department of Physics, Stockholm University, Sweden

${ }^{45 \mathrm{~b}}$ Oskar Klein Centre, Stockholm, Sweden

${ }^{46}$ Deutsches Elektronen-Synchrotron DESY, Hamburg and Zeuthen, Germany

${ }^{47}$ Lehrstuhl für Experimentelle Physik IV, Technische Universität Dortmund, Dortmund, Germany

${ }^{48}$ Institut für Kern- und Teilchenphysik, Technische Universität Dresden, Dresden, Germany

${ }^{49}$ Department of Physics, Duke University, Durham NC, United States of America

${ }^{50}$ SUPA - School of Physics and Astronomy, University of Edinburgh, Edinburgh, United Kingdom

${ }^{51}$ INFN e Laboratori Nazionali di Frascati, Frascati, Italy

${ }^{52}$ Physikalisches Institut, Albert-Ludwigs-Universität Freiburg, Freiburg, Germany

${ }^{53}$ II. Physikalisches Institut, Georg-August-Universität Göttingen, Göttingen, Germany

${ }^{54}$ Département de Physique Nucléaire et Corpusculaire, Université de Genève, Genève, Switzerland

${ }^{55}$ Dipartimento di Fisica, Università di Genova, Genova, Italy

${ }^{55 \mathrm{~b}}$ INFN Sezione di Genova, Italy

${ }^{56}$ II. Physikalisches Institut, Justus-Liebig-Universität Giessen, Giessen, Germany

${ }^{57}$ SUPA-School of Physics and Astronomy, University of Glasgow, Glasgow, United Kingdom

${ }^{58}$ LPSC, Université Grenoble Alpes, CNRS/IN2P3, Grenoble INP, Grenoble, France

${ }^{59}$ Laboratory for Particle Physics and Cosmology, Harvard University,

Cambridge MA, United States of America

${ }^{60 \mathrm{a}}$ Department of Modern Physics and State Key Laboratory of Particle Detection and Electronics, University of Science and Technology of China, Hefei, China

${ }^{60 \mathrm{~b}}$ Institute of Frontier and Interdisciplinary Science and Key Laboratory of Particle Physics and Particle Irradiation (MOE), Shandong University, Qingdao, China

${ }^{60 c}$ School of Physics and Astronomy, Shanghai Jiao Tong University, KLPPAC-MoE, SKLPPC, Shanghai, China

${ }^{60 \mathrm{~d}}$ Tsung-Dao Lee Institute, Shanghai, China

${ }^{61 \mathrm{a}}$ Kirchhoff-Institut für Physik, Ruprecht-Karls-Universität Heidelberg, Heidelberg, Germany

${ }^{61 \mathrm{~b}}$ Physikalisches Institut, Ruprecht-Karls-Universität Heidelberg, Heidelberg, Germany

${ }^{62}$ Faculty of Applied Information Science, Hiroshima Institute of Technology, Hiroshima, Japan

${ }^{63 a}$ Department of Physics, Chinese University of Hong Kong, Shatin, N.T., Hong Kong, China

${ }^{63 \mathrm{~b}}$ Department of Physics, University of Hong Kong, Hong Kong, China

${ }^{63 c}$ Department of Physics and Institute for Advanced Study, Hong Kong University of Science and

Technology, Clear Water Bay, Kowloon, Hong Kong, China

${ }^{64}$ Department of Physics, National Tsing Hua University, Hsinchu, Taiwan

${ }^{65}$ IJCLab, Université Paris-Saclay, CNRS/IN2P3, 91405, Orsay, France

${ }^{66}$ Department of Physics, Indiana University, Bloomington IN, United States of America

${ }^{67 a}$ INFN Gruppo Collegato di Udine, Sezione di Trieste, Udine, Italy

${ }^{67 \mathrm{~b}}$ ICTP, Trieste, Italy

${ }^{67 \mathrm{c}}$ Dipartimento Politecnico di Ingegneria e Architettura, Università di Udine, Udine, Italy

${ }^{68 \mathrm{a}}$ INFN Sezione di Lecce, Italy

${ }^{68 \mathrm{~b}}$ Dipartimento di Matematica e Fisica, Università del Salento, Lecce, Italy

${ }^{69}$ INFN Sezione di Milano, Italy

${ }^{69 \mathrm{~b}}$ Dipartimento di Fisica, Università di Milano, Milano, Italy

${ }^{70 a}$ INFN Sezione di Napoli, Italy

${ }^{70 \mathrm{~b}}$ Dipartimento di Fisica, Università di Napoli, Napoli, Italy

${ }^{71 \mathrm{a}}$ INFN Sezione di Pavia, Italy

${ }^{71 b}$ Dipartimento di Fisica, Università di Pavia, Pavia, Italy

${ }^{72 a}$ INFN Sezione di Pisa, Italy

${ }^{72 \mathrm{~b}}$ Dipartimento di Fisica E. Fermi, Università di Pisa, Pisa, Italy

${ }^{73 a}$ INFN Sezione di Roma, Italy

${ }^{73 b}$ Dipartimento di Fisica, Sapienza Università di Roma, Roma, Italy

${ }^{74 a}$ INFN Sezione di Roma Tor Vergata, Italy

${ }^{74 \mathrm{~b}}$ Dipartimento di Fisica, Università di Roma Tor Vergata, Roma, Italy

${ }^{75 a}$ INFN Sezione di Roma Tre, Italy

${ }^{75 b}$ Dipartimento di Matematica e Fisica, Università Roma Tre, Roma, Italy

${ }^{76 a}$ INFN-TIFPA, Italy

${ }^{76 \mathrm{~b}}$ Università degli Studi di Trento, Trento, Italy 
${ }^{77}$ Institut für Astro- und Teilchenphysik, Leopold-Franzens-Universität, Innsbruck, Austria

${ }^{78}$ University of Iowa, Iowa City IA, United States of America

${ }^{79}$ Department of Physics and Astronomy, Iowa State University, Ames IA, United States of America

${ }^{80}$ Joint Institute for Nuclear Research, Dubna, Russia

${ }^{81 a}$ Departamento de Engenharia Elétrica, Universidade Federal de Juiz de Fora (UFJF), Juiz de Fora, Brazil

${ }^{81 \mathrm{~b}}$ Universidade Federal do Rio De Janeiro COPPE/EE/IF, Rio de Janeiro, Brazil

${ }^{81 \mathrm{c}}$ Universidade Federal de São João del Rei (UFSJ), São João del Rei, Brazil

${ }^{81 \mathrm{~d}}$ Instituto de Física, Universidade de São Paulo, São Paulo, Brazil

${ }^{82}$ KEK, High Energy Accelerator Research Organization, Tsukuba, Japan

${ }^{83}$ Graduate School of Science, Kobe University, Kobe, Japan

${ }^{84 \mathrm{a}}$ AGH University of Science and Technology, Faculty of Physics and Applied Computer Science, Krakow, Poland

${ }^{84 \mathrm{~b}}$ Marian Smoluchowski Institute of Physics, Jagiellonian University, Krakow, Poland

${ }^{85}$ Institute of Nuclear Physics Polish Academy of Sciences, Krakow, Poland

${ }^{86}$ Faculty of Science, Kyoto University, Kyoto, Japan

${ }^{87}$ Kyoto University of Education, Kyoto, Japan

${ }^{88}$ Research Center for Advanced Particle Physics and Department of Physics, Kyushu University, Fukuoka, Japan

${ }^{89}$ Instituto de Física La Plata, Universidad Nacional de La Plata and CONICET, La Plata, Argentina

${ }^{90}$ Physics Department, Lancaster University, Lancaster, United Kingdom

${ }^{91}$ Oliver Lodge Laboratory, University of Liverpool, Liverpool, United Kingdom

${ }^{92}$ Department of Experimental Particle Physics, Jožef Stefan Institute and Department of Physics, University of Ljubljana, Ljubljana, Slovenia

${ }^{93}$ School of Physics and Astronomy, Queen Mary University of London, London, United Kingdom

${ }^{94}$ Department of Physics, Royal Holloway University of London, Egham, United Kingdom

${ }^{95}$ Department of Physics and Astronomy, University College London, London, United Kingdom

${ }^{96}$ Louisiana Tech University, Ruston LA, United States of America

${ }^{97}$ Fysiska institutionen, Lunds universitet, Lund, Sweden

${ }^{98}$ Centre de Calcul de l'Institut National de Physique Nucléaire et de Physique des Particules (IN2P3), Villeurbanne, France

${ }^{99}$ Departamento de Física Teorica C-15 and CIAFF, Universidad Autónoma de Madrid, Madrid, Spain

${ }^{100}$ Institut für Physik, Universität Mainz, Mainz, Germany

${ }^{101}$ School of Physics and Astronomy, University of Manchester, Manchester, United Kingdom

${ }^{102}$ CPPM, Aix-Marseille Université, CNRS/IN2P3, Marseille, France

${ }^{103}$ Department of Physics, University of Massachusetts, Amherst MA, United States of America

${ }^{104}$ Department of Physics, McGill University, Montreal QC, Canada

${ }^{105}$ School of Physics, University of Melbourne, Victoria, Australia

${ }^{106}$ Department of Physics, University of Michigan, Ann Arbor MI, United States of America

${ }^{107}$ Department of Physics and Astronomy, Michigan State University, East Lansing MI, United States of America

${ }^{108}$ B.I. Stepanov Institute of Physics, National Academy of Sciences of Belarus, Minsk, Belarus

${ }^{109}$ Research Institute for Nuclear Problems of Byelorussian State University, Minsk, Belarus

${ }^{110}$ Group of Particle Physics, University of Montreal, Montreal QC, Canada

${ }^{111}$ P.N. Lebedev Physical Institute of the Russian Academy of Sciences, Moscow, Russia

${ }^{112}$ National Research Nuclear University MEPhI, Moscow, Russia

${ }^{113}$ D.V. Skobeltsyn Institute of Nuclear Physics, M.V. Lomonosov Moscow State University, Moscow, Russia

${ }^{114}$ Fakultät für Physik, Ludwig-Maximilians-Universität München, München, Germany

${ }^{115}$ Max-Planck-Institut für Physik (Werner-Heisenberg-Institut), München, Germany

${ }^{116}$ Nagasaki Institute of Applied Science, Nagasaki, Japan

${ }^{117}$ Graduate School of Science and Kobayashi-Maskawa Institute, Nagoya University, Nagoya, Japan

${ }^{118}$ Department of Physics and Astronomy, University of New Mexico, Albuquerque NM, United States of America

${ }^{119}$ Institute for Mathematics, Astrophysics and Particle Physics, Radboud University Nijmegen/Nikhef, Nijmegen, Netherlands

${ }^{120}$ Nikhef National Institute for Subatomic Physics and University of Amsterdam, Amsterdam, Netherlands

${ }^{121}$ Department of Physics, Northern Illinois University, DeKalb IL, United States of America

${ }^{122 \mathrm{a}}$ Budker Institute of Nuclear Physics and NSU, SB RAS, Novosibirsk, Russia

${ }^{122 \mathrm{~b}}$ Novosibirsk State University Novosibirsk, Russia 
${ }^{123}$ Institute for High Energy Physics of the National Research Centre Kurchatov Institute, Protvino, Russia

${ }^{124}$ Institute for Theoretical and Experimental Physics named by A.I. Alikhanov of National Research Centre "Kurchatov Institute", Moscow, Russia

${ }^{125}$ Department of Physics, New York University, New York NY, United States of America

${ }^{126}$ Ochanomizu University, Otsuka, Bunkyo-ku, Tokyo, Japan

${ }^{127}$ Ohio State University, Columbus OH, United States of America

${ }^{128}$ Faculty of Science, Okayama University, Okayama, Japan

${ }^{129}$ Homer L. Dodge Department of Physics and Astronomy, University of Oklahoma, Norman OK, United States of America

${ }^{130}$ Department of Physics, Oklahoma State University, Stillwater OK, United States of America

${ }^{131}$ Palacký University, RCPTM, Joint Laboratory of Optics, Olomouc, Czech Republic

${ }^{132}$ Institute for Fundamental Science, University of Oregon, Eugene, OR, United States of America

${ }^{133}$ Graduate School of Science, Osaka University, Osaka, Japan

${ }^{134}$ Department of Physics, University of Oslo, Oslo, Norway

${ }^{135}$ Department of Physics, Oxford University, Oxford, United Kingdom

${ }^{136}$ LPNHE, Sorbonne Université, Université de Paris, CNRS/IN2P3, Paris, France

${ }^{137}$ Department of Physics, University of Pennsylvania, Philadelphia PA, United States of America

${ }^{138}$ Konstantinov Nuclear Physics Institute of National Research Centre "Kurchatov Institute”, PNPI, St. Petersburg, Russia

${ }^{139}$ Department of Physics and Astronomy, University of Pittsburgh, Pittsburgh PA, United States of America

${ }^{140 a}$ Laboratório de Instrumentação e Física Experimental de Partículas-LIP, Lisboa, Portugal

${ }^{140 \mathrm{~b}}$ Departamento de Física, Faculdade de Ciências, Universidade de Lisboa, Lisboa, Portugal

${ }^{140 \mathrm{c}}$ Departamento de Física, Universidade de Coimbra, Coimbra, Portugal

${ }^{140 \mathrm{~d}}$ Centro de Física Nuclear da Universidade de Lisboa, Lisboa, Portugal

${ }^{140 \mathrm{e}}$ Departamento de Física, Universidade do Minho, Braga, Portugal

${ }^{140 \mathrm{f}}$ Departamento de Física Teórica y del Cosmos, Universidad de Granada, Granada (Spain), Spain

${ }^{140 \mathrm{~g}}$ Dep Física and CEFITEC of Faculdade de Ciências e Tecnologia, Universidade Nova de Lisboa, Caparica, Portugal

${ }^{140 \mathrm{~h}}$ Instituto Superior Técnico, Universidade de Lisboa, Lisboa, Portugal

${ }^{141}$ Institute of Physics of the Czech Academy of Sciences, Prague, Czech Republic

${ }^{142}$ Czech Technical University in Prague, Prague, Czech Republic

${ }^{143}$ Charles University, Faculty of Mathematics and Physics, Prague, Czech Republic

${ }^{144}$ Particle Physics Department, Rutherford Appleton Laboratory, Didcot, United Kingdom

${ }^{145}$ IRFU, CEA, Université Paris-Saclay, Gif-sur-Yvette, France

${ }^{146}$ Santa Cruz, Institute for Particle Physics, University of California Santa Cruz, Santa Cruz CA, United States of America

${ }^{147 a}$ Departamento de Física, Pontificia Universidad Católica de Chile, Santiago, Chile

${ }^{147 \mathrm{~b}}$ Universidad Andres Bello, Department of Physics, Santiago, Chile

${ }^{147 \mathrm{c}}$ Instituto de Alta Investigación, Universidad de Tarapacá, Chile

${ }^{147 d}$ Departamento de Física, Universidad Técnica Federico Santa María, Valparaíso, Chile

${ }^{148}$ Department of Physics, University of Washington, Seattle WA, United States of America

${ }^{149}$ Department of Physics and Astronomy, University of Sheffield, Sheffield, United Kingdom

${ }^{150}$ Department of Physics, Shinshu University, Nagano, Japan

${ }^{151}$ Department Physik, Universität Siegen, Siegen, Germany

${ }^{152}$ Department of Physics, Simon Fraser University, Burnaby BC, Canada

${ }^{153}$ SLAC National Accelerator Laboratory, Stanford CA, United States of America

${ }^{154}$ Physics Department, Royal Institute of Technology, Stockholm, Sweden

${ }^{155}$ Departments of Physics and Astronomy, Stony Brook University,

Stony Brook NY, United States of America

${ }^{156}$ Department of Physics and Astronomy, University of Sussex, Brighton, United Kingdom

${ }^{157}$ School of Physics, University of Sydney, Sydney, Australia

${ }^{158}$ Institute of Physics, Academia Sinica, Taipei, Taiwan

${ }^{159 a}$ E. Andronikashvili Institute of Physics, Iv. Javakhishvili Tbilisi State University, Tbilisi, Georgia

${ }^{159 b}$ High Energy Physics Institute, Tbilisi State University, Tbilisi, Georgia

${ }^{160}$ Department of Physics, Technion, Israel Institute of Technology, Haifa, Israel

${ }^{161}$ Raymond and Beverly Sackler School of Physics and Astronomy, Tel Aviv University, Tel Aviv, Israel

${ }^{162}$ Department of Physics, Aristotle University of Thessaloniki, Thessaloniki, Greece

${ }^{163}$ International Center for Elementary Particle Physics and Department of Physics, University of Tokyo, Tokyo, Japan 


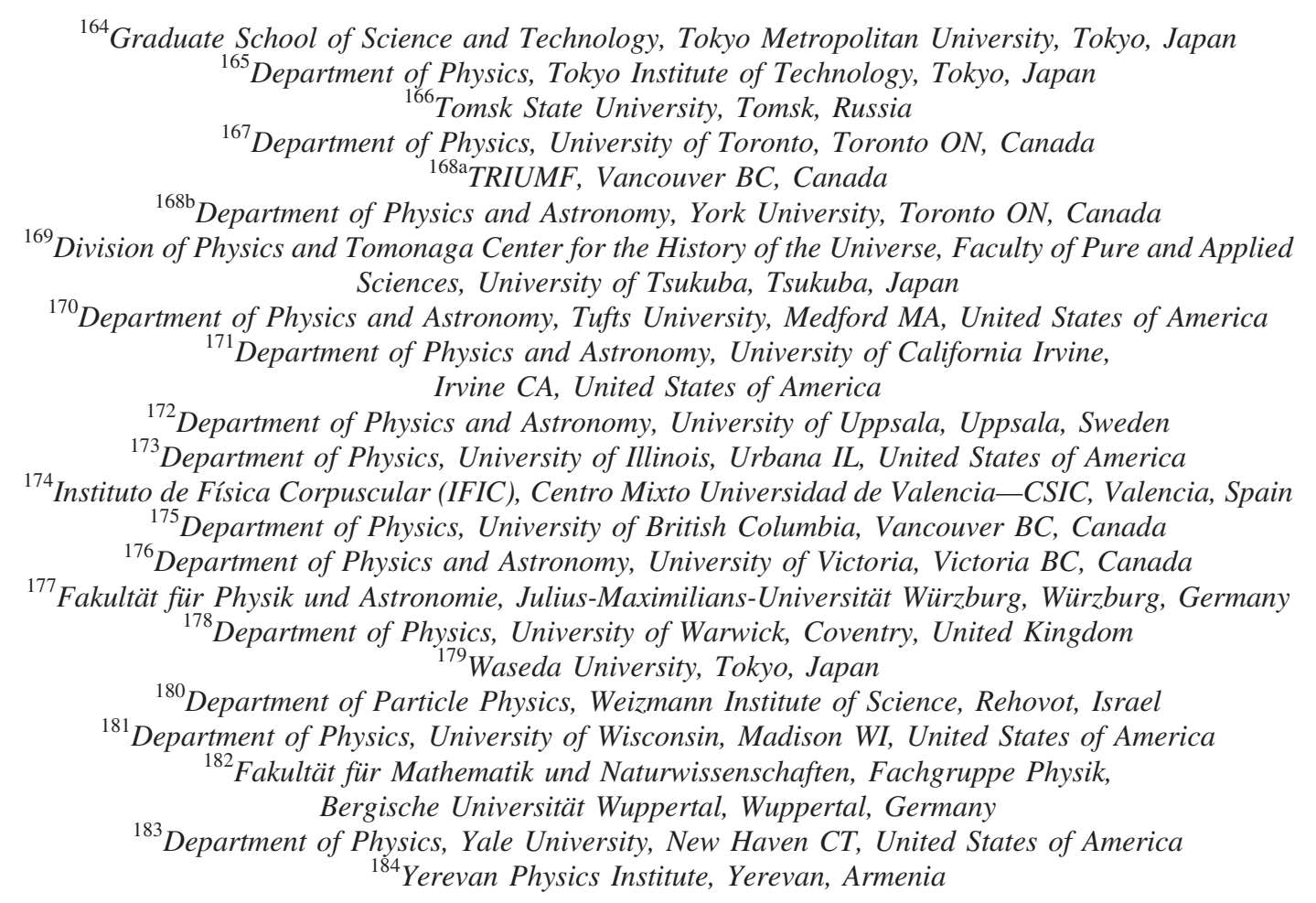

${ }^{\mathrm{a}}$ Deceased.

${ }^{\mathrm{b}}$ Also at Department of Physics, King's College London, London, United Kingdom

${ }^{\mathrm{c}}$ Also at Instituto de Fisica Teorica, IFT-UAM/CSIC, Madrid, Spain

${ }^{\mathrm{d}}$ Also at TRIUMF, Vancouver BC, Canada

${ }^{\mathrm{e}}$ Also at Department of Physics and Astronomy, University of Louisville, Louisville, KY, United States of America

${ }^{\mathrm{f}}$ Also at Physics Department, An-Najah National University, Nablus, Palestine

${ }^{g}$ Also at Department of Physics, California State University, Fresno, United States of America

${ }^{\mathrm{h}}$ Also at Department of Physics, University of Fribourg, Fribourg, Switzerland

${ }^{\mathrm{i}}$ Also at Physics Dept, University of South Africa, Pretoria, South Africa

${ }^{\mathrm{j}}$ Also at Departament de Fisica de la Universitat Autonoma de Barcelona, Barcelona, Spain

${ }^{k}$ Also at Moscow Institute of Physics and Technology State University, Dolgoprudny, Russia

${ }^{1}$ Also at The Collaborative Innovation Center of Quantum Matter (CICQM), Beijing, China

${ }^{\mathrm{m}}$ Also at Department of Physics, Ben Gurion University of the Negev, Beer Sheva, Israel

${ }^{\mathrm{n}}$ Also at Departamento de Física, Instituto Superior Técnico, Universidade de Lisboa, Lisboa, Portugal

${ }^{\circ}$ Also at Universita di Napoli Parthenope, Napoli, Italy

${ }^{\mathrm{p}}$ Also at Institute of Particle Physics (IPP), Vancouver, Canada

${ }^{\mathrm{q}}$ Also at Department of Physics, University of Adelaide, Adelaide, Australia

${ }^{\mathrm{r}}$ Also at Dipartimento di Matematica, Informatica e Fisica, Università di Udine, Udine, Italy

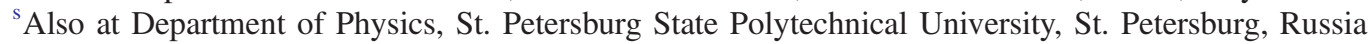

${ }^{\mathrm{t}}$ Also at Borough of Manhattan Community College, City University of New York, New York NY, United States of America

${ }^{u}$ Also at Department of Financial and Management Engineering, University of the Aegean, Chios, Greece

${ }^{v}$ Also at Department of Physics, California State University, East Bay, United States of America

${ }^{\mathrm{w}}$ Also at Institucio Catalana de Recerca i Estudis Avancats, ICREA, Barcelona, Spain

${ }^{\mathrm{x}}$ Also at Department of Physics, University of Michigan, Ann Arbor MI, United States of America

${ }^{y}$ Also at IJCLab, Université Paris-Saclay, CNRS/IN2P3, 91405, Orsay, France

${ }^{\mathrm{z}}$ Also at Graduate School of Science, Osaka University, Osaka, Japan

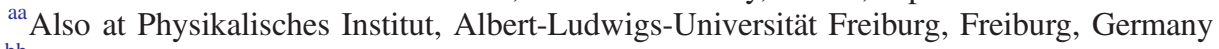

${ }^{\mathrm{bb}}$ Also at Institute of Physics, Azerbaijan Academy of Sciences, Baku, Azerbaijan

${ }^{c c}$ Also at Institute for Mathematics, Astrophysics and Particle Physics, Radboud University Nijmegen/Nikhef, Nijmegen, Netherlands

${ }^{\mathrm{dd}}$ Also at Institute of Theoretical Physics, Ilia State University, Tbilisi, Georgia

${ }^{\mathrm{ee}}$ Also at CERN, Geneva, Switzerland

${ }^{\mathrm{ff}}$ Also at Department of Physics, Stanford University, Stanford CA, United States of America

gg Also at Manhattan College, New York NY, United States of America

${ }^{\text {hh }}$ Also at Joint Institute for Nuclear Research, Dubna, Russia 
${ }^{\text {ii }}$ Also at Hellenic Open University, Patras, Greece

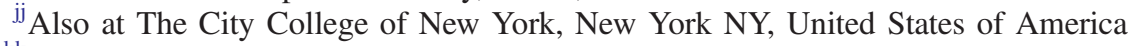

${ }^{k k}$ Also at Department of Physics, California State University, Sacramento, United States of America

${ }^{1 l}$ Also at Département de Physique Nucléaire et Corpusculaire, Université de Genève, Genève, Switzerland

${ }^{\mathrm{mm}}$ Also at Louisiana Tech University, Ruston LA, United States of America

${ }^{\mathrm{nn}}$ Also at School of Physics, Sun Yat-sen University, Guangzhou, China

${ }^{\circ o}$ Also at Institute for Nuclear Research and Nuclear Energy (INRNE) of the Bulgarian Academy of Sciences, Sofia, Bulgaria

${ }^{\mathrm{pp}}$ Also at Faculty of Physics, M.V. Lomonosov Moscow State University, Moscow, Russia

${ }^{\mathrm{qq}}$ Also at Department of Applied Physics and Astronomy, University of Sharjah, Sharjah, United Arab Emirates

${ }^{\mathrm{rr}}$ Also at Institut für Experimentalphysik, Universität Hamburg, Hamburg, Germany

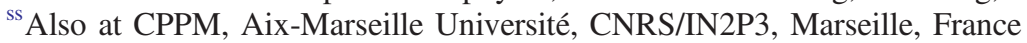

${ }^{\text {tt}}$ Also at National Research Nuclear University MEPhI, Moscow, Russia

${ }^{u u}$ Also at Institute for Particle and Nuclear Physics, Wigner Research Centre for Physics, Budapest, Hungary

${ }^{v v}$ Also at Giresun University, Faculty of Engineering, Giresun, Turkey

${ }^{w w}$ Also at Institute of Physics, Academia Sinica, Taipei, Taiwan

${ }^{\mathrm{xx}}$ Also at LPNHE, Sorbonne Université, Université de Paris, CNRS/IN2P3, Paris, France

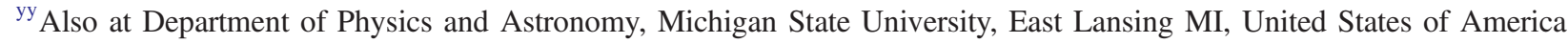

